CRUISE REPORT

SEA SEMESTER CLASS C-112

23 MAY - 3 JULY 1990

SCIENTIFIC ACTIVITIES UNDERTAKEN ABOARD SSV CORWITH CRAMER

Sea Education Association

Woods Hole, Massachusetts 
c

. 


\section{- TABLE OF CONTENTS}

Page

$\begin{array}{llr}\text { PART I INTRODUCTION } & 1\end{array}$

$\begin{array}{ll}\text { Cruise Narrative } & 10\end{array}$

$\begin{array}{lll}\text { PART II DATA } & 13\end{array}$

Surface Stations 13

Hydrostations $\quad 16$

CTD 19

Hydrocast $\quad 32$

Meter Net Tows $\quad 36$

Neuston Net Tows $\quad 37$

PART III SOME PRELIMINARY RESULTS

Horizontal Distributions from Surface Samples 38

Comparative Plankton Dynamics of Georges Bank and the Scotian Shelf

Georges Bank Tidal Front $\quad 45$

Gulf Stream Front $\quad 50$

Plastic and Tar in the Northwest Atlantic Ocean 57 



\section{PART I}

\section{INTRODUCTION}

This cruise report summarizes the academic activities aboard SSV Corwith Cramer during the Sea Education Association's SEA Semester Cruise C-112. The scientific program was designed to provide practical experience for college students in all fields of marine science and introduce them to a diverse group of marine habitats, from nearshore to deep sea. The students participated in all ship operations and presented results from a project of their own design. Eighteen students from fifteen colleges participated joining a crew of six nautical staff, four scientific staff, and one visiting scientist (Table I).

The cruise track, from Woods Hole - Bermuda - Nova Scotia - Woods Hole (Table II) was chosen to encompass a broad spectrum of oceanographic habitats ranging from New England and Nova Scotian continental shelves to the Sargasso Sea. Sampling included 39 CTD (conductivity-temperature-depth) profiles, 23 Niskin bottle casts, 26 meter net tows, 36 neuston net tows, over 70 surface stations and bathythermograph profiles and a few Shipek grabs and otter trawls. In addition, the academic program included lectures on various oceanographic subjects of general and regional interest (Table III). Particular areas of study were the tidal front north of Georges Bank, a comparison of the plankton dynamics on Georges Bank and the Scotian Shelf, and the Gulf Stream. The studies were written by the students as individual project reports (Table IV). The station locations and data collected are presented in Part II and selected results are presented in Part III. Not all the CTD data is presented but the rest is available on floppy disk.

I felt the cruise was highly successful because a large number of operations were completed and yielded valuable data with several interesting results. This is a tribute to the hard work and attention to detail of the entire ship's company--and I thank them. I would especially like to thank the Captain, Greg Lohse, for the extra effort he made to make the science program a success. 
Nautical Staff

Greg Lohse

Mark Crutcher

Laura Lohse

Brian Andrews

Robbie Laird

Laura Snyder

Scientific Staff

Richard Bohrer

Tim Kenna

Ed Lyman

Lee Thompson

Visiting Scientist

Otto Muller

Students

Julie Brown

Lisa Carter

Robert Deline

Kimberly Dent

Katherine Dwight

Amanda Goodpaster

Jonathan Harris

Nancy Israel

David Lamberger

Tamara Lange

Richard Lupia

Timothy Myrtle

Deirdre O'Mara

Robert Robertson

Benjamin Siegel

Renee Taylor

Rossanna Wang

Rebecca Wittenberg
Captain

First Mate

Second Mate

Third Mate

Engineer

Steward

Chief Scientist

First Assistant Scientist

Second Assistant Scientist

Third Assistant Scientist

Alfred University

Colorado College

Bowdoin College

St. Lawrence University

Wesleyan University

Yale University

Wellesley College

Mississippi State

Bard College

College of Wooster

Cornell University

University of Pennsylvania

University of California, Los Angeles

Princeton University

Hiram College

Colorado College

College of Charleston

Wellesley College

Bard College 
Table II. Cruise Track for SSV Corwith Cramer Cruise C-112

\begin{tabular}{|c|c|c|c|c|c|}
\hline STATION/POSITION & DATE & TIME & LOG (nm) & LATITUDE & LONGITUDE \\
\hline Depart & $5 / 23 / 90$ & 1810 & 0 & Woods Hole, MA & \\
\hline Anchor & $5 / 24 / 90$ & 0000 & $\mathbf{0}$ & Tarpaulin Cove, MA & \\
\hline SS 1 & $5 / 24 / 90$ & 1619 & 23.6 & $41^{\circ} 03.9^{\prime}$ & $70^{\circ} 44.4^{\prime}$ \\
\hline SS 2 & $5 / 24 / 90$ & 2143 & 43.8 & $40^{\circ} 52.8^{\prime}$ & $70^{\circ} 23.0^{\prime}$ \\
\hline SS 3 & $5 / 25 / 90$ & 0105 & 64.8 & $40^{\circ} 46.9^{\circ}$ & $70^{\circ} 85.4^{\prime}$ \\
\hline SS 4 & $5 / 25 / 90$ & 0510 & 89 & $40^{\circ} 41.0^{\prime}$ & $69^{\circ} 55.0^{\prime}$ \\
\hline SS 5 & $5 / 25 / 90$ & 1120 & 133.4 & $41^{\circ} 19.2^{\prime}$ & $69^{\circ} 03.1^{\prime}$ \\
\hline SS 6 & $5 / 25 / 90$ & 1858 & 157.0 & $41^{\circ} 33.1^{\prime}$ & $68^{\circ} 43.1^{\prime}$ \\
\hline SS 7 & $5 / 25 / 90$ & 2145 & 177.0 & $41^{\circ} 49.0^{\prime}$ & $68^{\circ} 27.1^{\prime}$ \\
\hline SS 8 & $5 / 26 / 90$ & 0426 & 212.1 & $42^{\circ} 04.3^{\prime}$ & $67^{\circ} 40.6^{\prime}$ \\
\hline SS 9 & $5 / 26 / 90$ & 0723 & 233.9 & $42^{\circ} 07.9^{\prime}$ & $67^{\circ} 10.5^{\prime}$ \\
\hline HC 4 & $5 / 26 / 90$ & 1105 & 247.5 & $42^{\circ} 15.0^{\prime}$ & $67^{\circ} 13.0^{\prime}$ \\
\hline $\mathrm{HC} 9$ & $5 / 26 / 90$ & 2240 & 272.3 & $41^{\circ} 57.4^{\prime}$ & $67^{\circ} 10.6^{\prime}$ \\
\hline HC 10 & $5 / 27 / 90$ & 0640 & 301.8 & $41^{\circ} 26.6^{\prime}$ & $67^{\circ} 13.2^{\prime}$ \\
\hline SS 10 & $5 / 28 / 90$ & 0100 & 340.0 & $41^{\circ} 07.1^{\prime}$ & $67^{\circ} 16.2^{\prime}$ \\
\hline HC 14 & $5 / 28 / 90$ & 0630 & 357.8 & $40^{\circ} 45.0^{\prime}$ & $67^{\circ 0} 8.2^{\prime}$ \\
\hline SS 11 & $5 / 28 / 90$ & 1200 & 370.2 & $40^{\circ} 27.2^{\prime}$ & $67^{\circ} 04.3^{\prime}$ \\
\hline SS 12 & $5 / 28 / 90$ & 1800 & 422.2 & $39^{\circ} 51.0^{\prime}$ & $67^{\circ} 10.8^{\prime}$ \\
\hline SS 13 & $5 / 28 / 90$ & 2143 & 442.9 & $39^{\circ} 32.7^{\prime}$ & $67^{\circ} 18.6^{\prime}$ \\
\hline SS 14 & $5 / 29 / 90$ & 0425 & 463.3 & $39^{\circ} 07.6^{\prime}$ & $67^{\circ} 28.6^{\prime}$ \\
\hline SS 15 & $5 / 29 / 90$ & 1314 & 488.5 & $38^{\circ} 42.7^{\prime}$ & $67^{\circ} 41.0^{\prime}$ \\
\hline SS 16 & $5 / 29 / 90$ & 1844 & 521 & $38^{\circ} 27.9^{\prime}$ & $68^{\circ} 02.5^{\prime}$ \\
\hline SS 17 & $5 / 29 / 90$ & 2330 & 546.3 & $38^{\circ} 12.3^{\prime}$ & $68^{\circ} 23.6^{\prime}$ \\
\hline SS 18 & $5 / 30 / 90$ & 0341 & 568.4 & $38^{\circ} 03.7^{\prime}$ & $68^{\circ} 01.4^{\prime}$ \\
\hline SS 19 & $5 / 30 / 90$ & 0745 & 591.2 & $37^{\circ} 48.6^{\prime}$ & $67^{\circ} 40.4^{\prime}$ \\
\hline Noon & $5 / 30 / 90$ & 1200 & 609.1 & $37^{\circ} 52.1^{\prime}$ & $67^{\circ} 17.2^{\prime}$ \\
\hline Midnight & $5 / 31 / 90$ & 0000 & 684.3 & $37^{\circ} 17.6^{\prime}$ & $66^{\circ} 21.7^{\prime}$ \\
\hline SS 20 & $5 / 31 / 90$ & 0740 & 715.3 & $37^{\circ} 01.4^{\prime}$ & $66^{\circ} 10.4^{\prime}$ \\
\hline SS 21 & $5 / 31 / 90$ & 1430 & 740.8 & $36^{\circ} 37.2^{\prime}$ & $65^{\circ} 56.8^{\prime}$ \\
\hline SS 22 & $5 / 31 / 90$ & 1905 & 762.0 & $36^{\circ} 16.0^{\prime}$ & $65^{\circ} 51.4^{\prime}$ \\
\hline SS 23 & $5 / 31 / 90$ & 2324 & 781.1 & $35^{\circ} 57.8^{\prime}$ & $65^{\circ} 49.8^{\prime}$ \\
\hline SS 24 & $6 / 01 / 90$ & 0619 & 801.3 & $35^{\circ} 42.3^{\prime}$ & $65^{\circ} 54.3^{\prime}$ \\
\hline SS 25 & $6 / 01 / 90$ & 1825 & 823.0 & $35^{\circ} 20.4^{\prime}$ & $65^{\circ} 53.5^{\prime}$ \\
\hline SS 26A & $6 / 01 / 90$ & 2359 & 851.0 & $34^{\circ} 57.8^{\prime}$ & $65^{\circ} 47.9^{\prime}$ \\
\hline Noon & $6 / 02 / 90$ & 1200 & 893.9 & $34^{\circ} 12.7^{\prime}$ & $65^{\circ} 44.7^{\prime}$ \\
\hline SS 26 & $6 / 02 / 90$ & 2100 & 926.5 & $33^{\circ} 39.8^{\prime}$ & $65^{\circ} 40.8^{\prime}$ \\
\hline SS 27 & $6 / 03 / 90$ & 0405 & 959.5 & $33^{\circ} 13.3^{\prime}$ & $65^{\circ} 18.3^{\prime}$ \\
\hline SS 28 & $6 / 03 / 90$ & 1000 & 990.7 & $32^{\circ} 47.3^{\prime}$ & $65^{\circ} 00.7^{\prime}$ \\
\hline SS 29 & $6 / 03 / 90$ & 1334 & 1010.6 & $32^{\circ} 44.3^{\prime}$ & $64^{\circ} 54.8^{\prime}$ \\
\hline SS 30 & $6 / 03 / 90$ & 1700 & 1023.3 & $32^{\circ} 56.5^{\prime}$ & $64^{\circ} 43.7^{\prime}$ \\
\hline SS 31 & $6 / 03 / 90$ & 2341 & 1046.7 & $32^{\circ} 39.1^{\prime}$ & $64^{\circ} 41.6^{\prime}$ \\
\hline Arrive & $6 / 04 / 90$ & 1000 & 1065.0 & St. George, Bermuda & \\
\hline Depart & $6 / 07 / 90$ & 0900 & 1065.0 & St. George, Bermuda & \\
\hline SS 32 & $6 / 07 / 90$ & 1251 & 1080.5 & $32^{\circ} 44.0^{\prime}$ & $64^{\circ} 51.8^{\prime}$ \\
\hline SS 33 & $6 / 07 / 90$ & 1645 & 1106.4 & $33^{\circ} 02.7$ & $64^{\circ} 34.6^{\prime}$ \\
\hline SS 34 & $6 / 07 / 90$ & 2030 & 1120.1 & $33^{\circ} 21.2^{\prime}$ & $64^{\circ} 32.9^{\prime}$ \\
\hline SS 35 & $6 / 08 / 90$ & 0239 & 1141.4 & $33^{\circ} 45.8^{\prime}$ & $64^{\circ} 30.7^{\prime}$ \\
\hline
\end{tabular}


Table II cont'd.

\begin{tabular}{|c|c|c|c|c|c|}
\hline STATION/POSITION & DATE & TIME & LOG (nm) & LATITUDE & LONGITUDE \\
\hline SS 36 & $6 / 08 / 90$ & 0500 & 1160.4 & $34^{\circ} 05.5^{\prime}$ & $64^{\circ} 29.1^{\prime}$ \\
\hline SS 37 & $6 / 08 / 90$ & 0807 & 1180.5 & $34^{\circ} 25.6^{\prime}$ & $64^{\circ} 27.8^{\prime}$ \\
\hline SS 38 & $6 / 08 / 90$ & 1300 & 1201.1 & $34^{\circ} 44.6^{\prime}$ & $64^{\circ} 33.1^{\prime}$ \\
\hline SS 39 & $6 / 08 / 90$ & 2245 & 1235.9 & $35^{\circ} 19.0^{\prime}$ & $64^{\circ} 46.0^{\prime}$ \\
\hline SS 40 & $6 / 09 / 90$ & 2051 & 1278.6 & $36^{\circ} 03.9^{\prime}$ & $64^{\circ} 46.4^{\prime}$ \\
\hline HC 32 & $6 / 09 / 90$ & 2315 & 1289.9 & $36^{\circ} 17.3$ & $64^{\circ} 48.2^{\prime}$ \\
\hline SS 41 & $6 / 10 / 90$ & 071.5 & 1331.2 & $37^{\circ} 02.1$ & $64^{\circ} 41.3^{\prime}$ \\
\hline SS 42 & $6 / 10 / 90$ & 1143 & 1353.2 & $37^{\circ} 22.9^{\prime}$ & $64^{\circ} 45.2$ \\
\hline SS 43 & $6 / 10 / 90$ & 1606 & 1378.8 & $37^{\circ} 48.9^{\prime}$ & $64^{\circ} 38.9^{\prime}$ \\
\hline SS 44 & $6 / 11 / 90$ & 0417 & 1399.9 & $38^{\circ} 08.4^{\prime}$ & $64^{\circ} 20.1^{\prime}$ \\
\hline SS 45 & $6 / 11 / 90$ & 0725 & 1419.7 & $38^{\circ} 22.0^{\prime}$ & $64^{\circ} 16.0^{\prime}$ \\
\hline SS 46 & $6 / 11 / 90$ & 1752 & 1441.1 & $38^{\circ} 29.4^{\prime}$ & $63^{\circ} 44.2^{\prime}$ \\
\hline HC 37 & $6 / 12 / 90$ & 0045 & 1459.9 & $38^{\circ} 40.2^{\prime}$ & $63^{\circ} 59.7^{\prime}$ \\
\hline HC 38 & $6 / 12 / 90$ & 1158 & 1488.5 & $38^{\circ} 50.1$ & $64^{\circ} 32.9^{\prime}$ \\
\hline HC 39 & $6 / 13 / 90$ & 2230 & 1587.6 & $39^{\circ} 10.0^{\prime}$ & $64^{\circ} 32.9^{\prime}$ \\
\hline SS 47 & $6 / 14 / 90$ & 0743 & 1606.6 & $39^{\circ} 20.7$ & $65^{\circ} 09.2$ \\
\hline $\mathrm{HC} 40$ & $6 / 14 / 90$ & 1030 & 1619.6 & $39^{\circ} 31.0^{\prime}$ & $65^{\circ} 04.6^{\prime}$ \\
\hline SS 48 & $6 / 14 / 90$ & 2300 & 1662.5 & $40^{\circ} 10.1^{\prime}$ & $65^{\circ} 05.4$ \\
\hline SS 49 & $6 / 15 / 90$ & 0251 & 1681.6 & $40^{\circ} 28.6^{\prime}$ & $64^{\circ} 56.1^{\prime}$ \\
\hline SS 50 & $6 / 15 / 90$ & 0348 & 1701.1 & $40^{\circ} 46.3^{\prime}$ & $64^{\circ} 54.5^{\prime}$ \\
\hline SS 51 & $6 / 15 / 90$ & 0925 & 1720.1 & $41^{\circ} 05.9^{\prime}$ & $64^{\circ} 48.4^{\prime}$ \\
\hline SS 52 & $6 / 15 / 90$ & 1500 & 1738.8 & $41^{\circ} 28.5^{\prime}$ & $64^{\circ} 43.7^{\prime}$ \\
\hline SS 53 & $6 / 15 / 90$ & 1855 & 1756.0 & $41^{\circ} 47.4^{\prime}$ & $64^{\circ} 35.3^{\prime}$ \\
\hline SS 54 & $6 / 15 / 90$ & 2000 & 1761.0 & $41^{\circ} 52.6^{\prime}$ & $64^{\circ} 32.9^{\prime}$ \\
\hline SS 55 & $6 / 15 / 90$ & 2205 & 1770.8 & $42^{\circ} 01.6^{\prime}$ & $64^{\circ} 28.5$ \\
\hline SS 56 & $6 / 16 / 90$ & 0450 & 1800.4 & $42^{\circ} 31.9^{\prime}$ & $64^{\circ} 19.5^{\prime}$ \\
\hline SS 57 & $6 / 17 / 90$ & 0000 & 1821.7 & $42^{\circ} 56.4^{\prime}$ & $64^{\circ} 18.5^{\prime}$ \\
\hline $\mathrm{HC} 48$ & $6 / 17 / 90$ & 0630 & 1834.6 & $43^{\circ} 10.2^{\prime}$ & $64^{\circ} 05.4^{\prime}$ \\
\hline SS 58 & $6 / 18 / 90$ & 0350 & 1858.1 & $43^{\circ} 32.2^{\prime}$ & $64^{\circ} 04.4^{\prime}$ \\
\hline SS 59 & $6 / 19 / 90$ & 0908 & 1880.1 & $43^{\circ} 54.3^{\prime}$ & $64^{\circ} 08.7^{\prime}$ \\
\hline Arrive & $6 / 19 / 90$ & 1000 & 1911.8 & \multicolumn{2}{|c|}{ Lunenberg, Nova Scotia } \\
\hline Depart & $6 / 21 / 90$ & 1705 & 1911.8 & \multicolumn{2}{|c|}{ Lunenberg, Nova Scotia } \\
\hline HC 52 & $6 / 21 / 90$ & 2305 & 1919.9 & $44^{\circ} 11.4^{\prime}$ & $64^{\circ} 09.9^{\prime}$ \\
\hline SS 60 & $6 / 22 / 90$ & 0310 & 1942.7 & $43^{\circ} 50.7^{\prime}$ & $64^{\circ} 26.0^{\prime}$ \\
\hline SS 61 & $6 / 22 / 90$ & 0729 & 1961.0 & $43^{\circ} 32.3^{\prime}$ & $64^{\circ} 31.3$ \\
\hline SS 62 & $6 / 22 / 90$ & 1231 & 1980.8 & $43^{\circ} 09.2^{\prime}$ & $64^{\circ} 31.1$ \\
\hline SS 63 & $6 / 22 / 90$ & 1920 & 2000.0 & $43^{\circ} 18.9^{\prime}$ & $64^{\circ} 56.2$ \\
\hline SS 64 & $6 / 23 / 90$ & 1115 & 2022.4 & $43^{\circ} 03.3^{\prime}$ & $65^{\circ} 16.9$ \\
\hline SS 65 & $6 / 23 / 90$ & 1540 & 2059.6 & $42^{\circ} 43.0^{\prime}$ & $65^{\circ} 55.8$ \\
\hline SS 66 & $6 / 23 / 90$ & 2130 & 2079.1 & $42^{\circ} 39.9^{\prime}$ & $66^{\circ} 27.7$ \\
\hline SS 67 & $6 / 24 / 90$ & 0035 & 2102.0 & $42^{\circ} 39.9^{\prime}$ & $67^{\circ} 00.8^{\prime}$ \\
\hline SS 68 & $6 / 24 / 90$ & 0350 & 2123.1 & $42^{\circ} 38.3^{\prime}$ & $67^{\circ} 25.6^{\prime}$ \\
\hline SS 69 & $6 / 24 / 90$ & 0800 & 2141.4 & $42^{0} 38.5^{\prime}$ & $67^{\circ} 48.0^{\prime}$ \\
\hline SS 70 & $6 / 24 / 90$ & 1250 & 2161.5 & $42^{\circ} 39.7$ & $68^{\circ} 18.8^{\prime}$ \\
\hline SS 71 & $6 / 24 / 90$ & 1847 & 2181.9 & $42^{\circ} 41.0^{\prime}$ & $68^{\circ} 48.0^{\prime}$ \\
\hline SS 72 & $6 / 24 / 90$ & 2215 & 2205.4 & $42^{\circ} 44.8^{\prime}$ & $69^{\circ} 19.6$ \\
\hline SS 73 & $6 / 25 / 90$ & 0220 & 2220.2 & $42^{\circ} 51.1^{\prime}$ & $69^{\circ} 35.4^{\prime}$ \\
\hline SS 74 & $6 / 25 / 90$ & 0745 & 2239.3 & $42^{\circ} 56.4^{\prime}$ & $70^{\circ} 00.6^{\prime}$ \\
\hline SS 75 & $6 / 25 / 90$ & 1320 & 2261.2 & $42^{\circ} 52.8^{\prime}$ & $70^{\circ} 08.9$ \\
\hline SS 76 & $6 / 26 / 90$ & 0030 & 2283.1 & $42^{\circ} 37.7^{\prime}$ & $69^{\circ} 43.4^{\prime}$ \\
\hline SS 77 & $6 / 26 / 90$ & 0350 & 2299.9 & $42^{\circ} 39.8^{\prime}$ & $70^{\circ} 03.2^{\prime}$ \\
\hline SS 78 & $6 / 26 / 90$ & 1145 & 2322.2 & $42^{\circ} 35.9^{\prime}$ & $70^{\circ} 17.9^{\prime}$ \\
\hline
\end{tabular}


Table II cont'd.

$\begin{array}{lrrrll}\text { STATION/POSITION } & \text { DATE } & \text { TIME } & \text { LOG (nm) } & \text { LATITUDE } & \text { LONGITUDE } \\ \text { Arrive } & 6 / 26 / 90 & 1500 & 2337.5 & \text { Gloucester, MA } & \\ \text { Depart } & 6 / 26 / 90 & 1840 & 23375 & \text { Gloucester, MA } & \\ \text { HC 62 } & 6 / 26 / 90 & 2100 & 2347.4 & 42^{\circ} 35.6^{\prime} & 7^{\circ} 29.3^{\prime} \\ \text { HC } 64 & 6 / 27 / 90 & 0420 & 2370.4 & 42^{\circ} 34.0^{\prime} & 0^{\circ} 013^{\prime} \\ \text { HC 66 } & 6 / 27 / 90 & 1140 & 2398.6 & 42^{\circ} 36.9^{\prime} & 9^{\circ} 33.4^{\prime} \\ \text { HC 69 } & 6 / 29 / 90 & 0500 & 2521.1 & 42^{\circ} 21.4^{\prime} & 7^{\circ} 16.9^{\prime} \\ \text { Arrive } & 6 / 30 / 90 & 0930 & 2569.6 & \text { Isles of Shoals, NH } & \\ \text { Depart } & 7 / 01 / 90 & 0700 & 2569.6 & \text { Isles of Shoals, NH } & \\ \text { Enter Cape Cod Canal } & 7 / 02 / 90 & 0845 & 2660.8 & 41^{\circ} 46.6^{\prime} & 70^{\circ} 29.5^{\prime} \\ \text { Anchor } & 7 / 03 / 90 & 0000 & 2696.4 & \text { Tarpaulin Cove, MA } & \\ \text { Arrive } & 7 / 03 / 90 & 0900 & 2700.5 & \text { Woods Hole, MA } & \end{array}$


Table III. Academic Program aboard SSV Corwith Cramer C-112

Date $\quad$ Topic

$5 / 24 / 90 \quad$ The Bathythermograph

$5 / 25 / 90 \quad$ Neuston Tows and Hydrocasts

$5 / 28 / 90 \quad$ Plankton Modeling of Georges Bank

$5 / 29 / 90 \quad$ Modeling Nutrient Recycling

5/30/90 Spectrophotometry

5/31/90 Oxygen Analysis

5/31/90 Gulf Stream Meanders

6/01/90 Practical Exam

6/07/90 Salinity Analysis and Fluorometry.

6/08/90 Gulf Stream Rings

6/11/90 Crustaceans

6/12/90 The Kelvin Seamounts and the Opening of the Atlantic

$6 / 13 / 90$

$6 / 14 / 90$

$6 / 15 / 90$

$6 / 18 / 90$

$6 / 22 / 90$

$6 / 25 / 90$

$6 / 26-29 / 90$ $7 / 01 / 90$
The PDR and Cetacean Acoustics

Buoyancy in Marine Organisms

Sea Level Fluctuations and the Supercycle Theory

Student Presentations of Selected Oceanographic Stations

Vertical Migration

Hagfish and Fish Evolution

Student Project Presentation

Final Exam
Lecturer

Tim Kenna

All Staff

Richard Bohrer

Richard Bohrer

Lee Thompson

Tim Kenna and

Ed Lyman

Richard Bohrer

Tim Kenna

Richard Bohrer

Tim Kenna

Otto Muller

Lee Thompson

Ed Lyman

Otto Muller

Richard Bohrer

Ed Lyman and

Tim Kenna 
Table IV. Student Projects aboard C-112

A Study of Competition between Benthic Fish on George's Bank through Analysis of Stomach Contents

The Effect of Fronts on Plastic Distribution in the Western North Atlantic Ocean

The Relationship between Surface Bioluminescence and Near-surface Mesoplankton of the Western North Atlantic

Chlorophyll $a$ and Two Frontal Zones: A Study of Biomass Kimberly Dent Relation to Frontal Currents

Current Speed and Direction on the Northern Flank of Georges Bank

Community Grazing and Diel Feeding Patterns of Zooplankton on Georges Bank and the Nova Scotian Shelf

The Species-Area Relationship of the Sargassum Community

Project Tar

Nutrient Regeneration by Zooplankton Excretion and Its Contribution to Phytoplankton Growth Requirements on Georges Bank and the Scotian Shelf

Calculation of the Velocity of the Gulf Stream Using A Model of Geostrophic Flow

Olfactory Foraging in Procellariiformes: Responses to Fish Oil and Chicken Fat

Primary Production on Georges Bank and the Scotian Shelf

The Relationship between Abundance of Off-Shore and Pelagic Marine Bird Species and Surface Physical Oceanographic Characteristics

Production of Sargassum natans in Relation to Macrofaunal Coverage

Julie Brown

Lisa Carter

Robert DeLine

Katie Dwight

Nancy Israel

David Lamberger

Tamara Lange

Richard Lupia

Timothy Myrtle

Deirdre O'Mara

Robert Robertson

Ben Siegel

Renee Taylor 
A Comparative Description of the Bermudian and Scotian Shelf/Slope Systems with an Emphasis on Grain Size Distribution as Related to Depth

Zooplankton Biomass across Fronts and Its Relationship to Whale Sightings

Observational Weather Predictions Based on Folklore
Jonathan Grant Harris

Rosanna Wang

Rebecca Whittenberg 


\section{CRUISE NARRATIVE}

It had been a beautiful sunny spring in Woods Hole but the weekend before we left we had a cold snap. This was just as well as it reminded us to take some warm clothes on our trip since Georges Bank, our first destination, can be quite cold at the end of May.

We boarded on Wednesday, May 23 and spent the first night and next morning in orientation and drills. After leaving, we had light winds and motor-sailed toward Georges Bank. We did three stations on the way there and on Saturday we did our tidal front transect of the northern flank of Georges Bank. We did six stations in 13 hours! Good job. The next day we did a 24-hour station in the center of the Bank. The weather was beautiful and the water almost glassy calm. We planned on measuring primary productivity in situ by hanging bottles from a buoy. After we had everything set up and were ready to toss it overboard, Mark suggested attaching another float. Good idea! As we cheered the line sinking down, we watched the buoy and radar reflector get sucked under with only the extra float remaining above water. After a recovery and the removal of some weight, the string of bottles was successfully launched. This was not to be the end of our problem, however. Finding the buoy at the end of the day proved difficult as the tides had moved it over a mile. Ed finally saw it from the mast around sunset and it was brought aboard.

The next morning we did our second otter trawl and began sailing off the bank. As everyone was crowded around the catch, Robbie came up to me and said it seemed to be getting much warmer. Soon, people were stripping off clothes as the water temperature went from $8^{\circ} \mathrm{C}$ to $20^{\circ} \mathrm{C}$ in less than 10 miles. The water became a clear blue and we began seeing flying fish and Sargassum. It was as if we had been teleported the six hundred miles to Bermuda in an instant. What it was, of course, was a warm core eddy nestled up alongside Georges Bank. We were all happy to put away our warm clothes as we headed for the Gulf Stream.

One week after boarding, we hit the Gulf Stream or, more appropriately, it hit us. Now, the Gulf Stream is very energetic both kinetically and thermodynamically so crossing can be rough. Our crossing proved to be so as we experienced the first heavy weather of the trip. We also blew out the mainstaysail in a squall. Greg had seen it coming and ordered the jib taken down but, as that was being done the mainstaysail blew (not something you would normally expect but it was an old one). After a rough night we entered the Sargasso Sea and did a hydrocast to $3000 \mathrm{~m}$. On Saturday, Greg had a sail handling class followed by a swim call and swizzle where gear adrift was auctioned off for future entertainment (Rob R. leading a Jane Fonda workout, Laura S. with songs, Katie and Ed snarfling spaghetti, Tim K. and Mark with a skit). On Monday, after doing some Shipek grabs on the way in, we arrived in Bermuda Harbour where we received bad news from a Canadian naval vessel that the Bruins had lost the Stanley Cup finals. 
We were able to dock right off the main square in St. George and proceeded to enjoy ourselves. Leave was spent in snorkeling at the bay across the island, traveling the island by bus or moped or motorcycle (Rob D. doing wheelies) or golf cart (Tim K. and Mark), playing pool, dancing, or just visiting the Whitehorse. Watches were spent mending sail or doing chemistry. When we departed, Greg showed his stuff by taking us off the dock under sail.

Underway again, we got our sea legs back and on Saturday had field day followed by our second swim call. During class in the afternoon, we hit a big patch of Sargassum. As we prepared to do a neuston tow, Lee got a strike on his fishing line. What ensued was a classic, titanic struggle between man and fish worthy of Hemingway. With all hands crowded round to watch, Lee worked to bring the fish in. It leapt out of the water once or twice, revealing itself as a $\mathbf{3 0}$ pound mahi-mahi. After over an hour it was landed and became supper that night for the whole ship. The next day we began our second encounter with the Gulf Stream.

We intended to do a transect of stations across the Gulf Stream and for the first 24 hours, all went well. Then the wind shifted and we had to alter course. Unfortunately, our new course had us trying to go upstream. Since the Gulf Stream velocity is $4 \mathrm{kn}$ and our usual sailing speed is also $4 \mathrm{kn}$, we made slow to no progress. It then got so rough that we had to heave to for a while during which we got blown backwards. It was 2-1/2 days later when we finally escaped the clutches of the Stream. Besides getting a nice adrenalin rush, we were able to complete all our stations and about ten more mahi-mahi which were caught from a school of juveniles at one station in about an hour.

Two days later we hit the fog of the Scotian Shelf and brought back out the warm clothes. The next day we did our second 24-hour station but didn't use the buoy as it was too foggy. Instead, we hung the bottles from the hydrowire. We did a series of neuston tows at night to look at the timing of migration and then headed for Lunenberg where we anchored in Rose Bay the night before going in. Lunenberg was a quieter stop than Bermuda as people were busy working on their projects. Some people got out to the countryside while most just visited the schooner museum and the local pubs. After three days we headed back out into the fog.

A blown board in the winch caused us to head directly to Gloucester for a replacement. On the way there, we did a ship's mission to Brown's Bank (Rob D.: "I feel the need for speed") and a BT series across the Gulf of Maine. After fixing the winch, we did a study of the low salinity water found off Cape Ann which is associated with blooms of toxic dinoflagellates. One night the watches had a sailing competition in which they got points for different sailing maneuvers while keeping the ship within a "box" described on the chart. We also put out traps for hagfish and were successful on the third attempt. After playing with the slime, the hagfish were stored in a cooler for delivery to researchers at the Isle of Shoals despite a mock protest by Greg and Laura S. that we "Save the Slime Hag". While all this was happening, the students presented their projects. Lots of good work was done as is evident by some of the results presented 
later in this report. On our last weekend we visited the Shoals Marine Laboratory. We had a cookout on shore and received lectures from some of their scientists (Who can forget Rick Martini's shark lecture?)

We came back to Woods Hole through the Cape Cod Canal and had to tack down a blowy Buzzards Bay before anchoring in Tarpaulin Cove for our final party. Ben's parents met us there and came over for a visit. The next morning we sailed to Woods Hole and our trip was at an end. 
PART II. Data

Table V. Surface Stations and Data for SSV Corwith Cramer Cruise C-112.

\begin{tabular}{|c|c|c|c|c|c|c|c|c|c|c|}
\hline SS\# & Date & Time & BT\# & $\log (\mathrm{nm})$ & Latitude & Longitude & $\%$ Sal. & $\mathrm{T}_{\mathrm{s}}$ & Chl. & $\mathrm{PO}_{4}$ \\
\hline 1 & $5 / 24 / 90$ & 1619 & 1 & 23.6 & $41^{\circ} 03.9^{\prime} \mathrm{N}$ & $70^{\circ} 44.4^{\prime} \mathrm{W}$ & 33.03 & $9.4^{\circ} \mathrm{C}$ & .83 & .76 \\
\hline 2 & $5 / 24 / 90$ & 2143 & 2 & 43.8 & $40^{\circ} 52.8^{\prime} \mathrm{N}$ & $70^{\circ} 22.0^{\prime} \mathrm{W}$ & 32.77 & $9.3^{\circ} \mathrm{C}$ & .51 & .42 \\
\hline 3 & $5 / 25 / 90$ & 0105 & -- & 64.8 & $40^{\circ} 46.9^{\prime} \mathrm{N}$ & $70^{\circ} 05.4^{\prime} \mathrm{W}$ & 32.76 & $8.6^{\circ} \mathrm{C}$ & .63 & .67 \\
\hline 4 & $5 / 25 / 90$ & 0510 & 3 & 89.0 & $40^{\circ} 41.0^{\prime} \mathrm{N}$ & $69^{\circ} 55.0^{\prime} \mathrm{W}$ & 32.81 & $7.4^{\circ} \mathrm{C}$ & 1.43 & 1.84 \\
\hline 5 & $5 / 25 / 90$ & 1120 & 4 & 133.4 & $41^{\circ} 19.2^{\prime} \mathrm{N}$ & $69^{\circ} 03.1^{\prime} \mathrm{W}$ & 32.65 & $8.7^{\circ} \mathrm{C}$ & .50 & .44 \\
\hline 6 & $5 / 25 / 90$ & 1858 & 5 & 157.0 & $41^{\circ} 33.1^{\prime} \mathrm{N}$ & $68^{\circ} 43.1^{\prime} \mathrm{W}$ & 32.90 & $8.4^{\circ} \mathrm{C}$ & .25 & .69 \\
\hline 7 & $5 / 25 / 90$ & 2145 & 6 & 177.0 & $41^{\circ} 49.0^{\prime} \mathrm{N}$ & $68^{\circ} 27.1^{\prime} \mathrm{W}$ & 32.84 & $8.2^{\circ} \mathrm{C}$ & .68 & .71 \\
\hline 8 & $5 / 26 / 90$ & 0420 & 7 & 212.1 & $42^{\circ} 04.3^{\prime} \mathrm{N}$ & $67^{\circ} 40.6^{\prime} \mathrm{W}$ & 32.82 & $7.7^{\circ} \mathrm{C}$ & .52 & 1.16 \\
\hline 9 & $5 / 26 / 90$ & 0723 & 8 & 233.9 & $42^{\circ} 07.9^{\prime} \mathrm{N}$ & $67^{\circ} 10.5^{\prime} \mathrm{W}$ & 32.99 & $7.3^{\circ} \mathrm{C}$ & -- & .37 \\
\hline -- & $5 / 26 / 90$ & 0814 & 9 & 237.9 & $42^{\circ} 04.2^{\prime} \mathrm{N}$ & $67^{\circ} 10.6^{\prime} \mathrm{W}$ & -- & $7.0^{\circ} \mathrm{C}$ & -- & -- \\
\hline -- & $5 / 26 / 90$ & 1310 & 10 & 251.0 & $42^{\circ} 11.0^{\prime} \mathrm{N}$ & $67^{\circ} 12.5^{\prime} \mathrm{W}$ & -- & $8.4^{\circ} \mathrm{C}$ & - & - \\
\hline - & $5 / 27 / 90$ & 0523 & 11 & 301.8 & $41^{\circ} 29.5^{\prime} \mathrm{N}$ & $67^{\circ} 11.3^{\prime} \mathrm{W}$ & -- & $8.2^{\circ} \mathrm{C}$ & -- & $=$ \\
\hline 10 & $5 / 28 / 90$ & 0100 & 12 & 340.0 & $41^{\circ} 07.1^{\prime} \mathrm{N}$ & $67^{\circ} 16.2^{\prime} \mathrm{W}$ & 33.21 & $8.1^{\circ} \mathrm{C}$ & .74 & .55 \\
\hline 11 & $5 / 28 / 90$ & 1200 & 13 & 370.2 & $40^{\circ} 29.3^{\prime} \mathrm{N}$ & $67^{\circ} 04.3^{\prime} \mathrm{W}$ & 36.42 & $20.3^{\circ} \mathrm{C}$ & .14 & .79 \\
\hline 12 & $5 / 28 / 90$ & 1800 & 14 & 422.2 & $39^{\circ} 51.0^{\prime} \mathrm{N}$ & $67^{\circ} 10.8^{\prime} \mathrm{W}$ & 36.10 & $22.1^{\circ} \mathrm{C}$ & .18 & .97 \\
\hline 13 & $5 / 28 / 90$ & 2143 & 15 & 442.9 & $39^{\circ} 32.7^{\prime} \mathrm{N}$ & $67^{\circ} 18.6^{\prime} \mathrm{W}$ & 35.50 & $19.4^{\circ} \mathrm{C}$ & 1.38 & .14 \\
\hline 14 & $5 / 29 / 90$ & 0425 & 16 & 463.3 & $39^{\circ} 07.6^{\prime} \mathrm{N}$ & $67^{\circ} 28.6^{\prime} \mathrm{W}$ & 34.99 & $18.7^{\circ} \mathrm{C}$ & .25 & .52 \\
\hline 15 & $5 / 29 / 90$ & 1314 & 17 & 488.5 & $38^{\circ} 42.7^{\prime} \mathrm{N}$ & $67^{\circ} 41.0^{\prime} \mathrm{W}$ & 34.80 & $18.9^{\circ} \mathrm{C}$ & .17 & .16 \\
\hline 16 & $5 / 29 / 90$ & 1844 & 18 & 521.0 & $38^{\circ} 27.9^{\prime} \mathrm{N}$ & $68^{\circ} 02.5^{\prime} \mathrm{W}$ & 34.93 & $18.7^{\circ} \mathrm{C}$ & .13 & -.01 \\
\hline 17 & $5 / 29 / 90$ & 2330 & 19 & 546.3 & $38^{\circ} 12.3^{\prime} \mathrm{N}$ & $68^{\circ} 23.6^{\prime} \mathrm{W}$ & 35.35 & $20.5^{\circ} \mathrm{C}$ & .13 & .11 \\
\hline 18 & $5 / 30 / 90$ & 0341 & 20 & 568.4 & $38^{\circ} 03.7^{\prime} \mathrm{N}$ & $68^{\circ} 01.4^{\prime} \mathrm{W}$ & 35.04 & $18.7^{\circ} \mathrm{C}$ & .22 & .49 \\
\hline 19 & $5 / 30 / 90$ & 0745 & -- & 591.2 & $37^{\circ} 48.6^{\prime} \mathrm{N}$ & $67^{\circ} 40.4^{\prime} \mathrm{W}$ & 35.18 & $19.3^{\circ} \mathrm{C}$ & .17 & -- \\
\hline$\cdot 20$ & $5 / 31 / 90$ & 0740 & 21 & 715.3 & $37^{\circ} 01.4^{\prime} \mathrm{N}$ & $66^{\circ} 10.4^{\prime} \mathrm{W}$ & 36.56 & $22.7^{\circ} \mathrm{C}$ & .10 & .16 \\
\hline 21 & $5 / 31 / 90$ & 1430 & 22 & 740.8 & $36^{\circ} 37.2^{\prime} \mathrm{N}$ & $65^{\circ} 56.8^{\prime} \mathrm{W}$ & 36.85 & $22.9^{\circ} \mathrm{C}$ & .09 & .11 \\
\hline 22 & $5 / 31 / 90$ & 1905 & 23 & 762.0 & $36^{\circ} 16.0^{\prime} \mathrm{N}$ & $65^{\circ} 51.4^{\prime} \mathrm{W}$ & 36.71 & $23.3^{\circ} \mathrm{C}$ & .07 & .07 \\
\hline 23 & $5 / 31 / 90$ & 2324 & - & 781.1 & $35^{\circ} 57.8^{\prime} \mathrm{N}$ & $65^{\circ} 49.8^{\prime} \mathrm{W}$ & 36.60 & $22.8^{\circ} \mathrm{C}$ & .09 & .39 \\
\hline 24 & $6 / 01 / 90$ & 0619 & 24 & 801.3 & $35^{\circ} 42.3^{\prime} \mathrm{N}$ & $65^{\circ} 54.3^{\prime} \mathrm{W}$ & 36.82 & $21.3^{\circ} \mathrm{C}$ & .03 & .09 \\
\hline 25 & $6 / 01 / 90$ & 1825 & 25 & 823.0 & ${ }^{\circ} 20.4 ' \mathrm{~N}$ & $65^{\circ} 53.5^{\prime} \mathrm{W}$ & 36.76 & $21.7^{\circ} \mathrm{C}$ & .02 & -.08 \\
\hline $26 \mathrm{~A}$ & $6 / 01 / 90$ & 2359 & -- & 851.0 & $34^{\circ} 57.8^{\prime} \mathrm{N}$ & $65^{\circ} 47.9^{\prime} \mathrm{W}$ & 36.91 & $21.7^{\circ} \mathrm{C}$ & .00 & -.05 \\
\hline 26 & $6 / 02 / 90$ & 2100 & 26 & 926.5 & $33^{\circ} 39.8^{\prime} \mathrm{N}$ & $65^{\circ} 40.8^{\prime} \mathrm{W}$ & 36.68 & $22.1^{\circ} \mathrm{C}$ & .05 & .27 \\
\hline 27 & $6 / 03 / 90$ & 0405 & 27 & 959.5 & $33^{\circ} 13.3^{\prime} \mathrm{N}$ & $65^{\circ} 18.3^{\prime} \mathrm{W}$ & 36.54 & $21.6^{\circ} \mathrm{C}$ & .03 & .04 \\
\hline 28 & $6 / 03 / 90$ & 1000 & 28 & 990.7 & $32^{\circ} 47.3^{\prime} \mathrm{N}$ & $65^{\circ} 00.7^{\prime} \mathrm{W}$ & 36.66 & $22.2^{\circ} \mathrm{C}$ & .04 & .16 \\
\hline 29 & $6 / 03 / 90$ & 1334 & 29 & 1010.6 & $44.3^{3} \mathrm{~N}$ & $64^{\circ} 54.8^{\prime} \mathrm{W}$ & 36.80 & $22.3^{\circ} \mathrm{C}$ & .01 & .09 \\
\hline 30 & $6 / 03 / 90$ & 1700 & -- & 1023.3 & $56.5 \mathrm{~N}$ & $64^{\circ} 43.7^{\prime} \mathrm{W}$ & 36.54 & $22.3^{\circ} \mathrm{C}$ & .00 & -.03 \\
\hline 31 & $6 / 03 / 90$ & 2341 & 30 & 1046.7 & $32^{\circ} 39.1^{\prime} \mathrm{N}$ & $64^{\circ} 41.6^{\prime} \mathrm{W}$ & 36.46 & $22.0^{\circ} \mathrm{C}$ & .04 & -- \\
\hline 32 & $6 / 07 / 90$ & 1251 & 31 & 1080.5 & $32^{\circ} 44.0^{\prime} \mathrm{N}$ & $64^{\circ} 51.8^{\prime} \mathrm{W}$ & 36.53 & $23.3^{\circ} \mathrm{C}$ & .04 & . 11 \\
\hline 33 & $6 / 07 / 90$ & 1645 & 32 & 1106.4 & $33^{\circ} 02.7^{\prime} \mathrm{N}$ & $64^{\circ} 34.6^{\prime} \mathrm{W}$ & 36.60 & $23.7^{\circ} \mathrm{C}$ & .04 & .06 \\
\hline 34 & $6 / 07 / 90$ & 2030 & 33 & 1120.1 & $33^{\circ} 21.2^{\prime} \mathrm{N}$ & $64^{\circ} 32.9^{\prime} \mathrm{W}$ & 36.38 & $23.4^{\circ} \mathrm{C}$ & .04 & .02 \\
\hline 35 & $6 / 08 / 90$ & 0239 & 34 & 1141.4 & $33^{\circ} 45.8^{\prime} \mathrm{N}$ & $64^{\circ} 30.7>\mathrm{W}$ & 36.71 & $23.2^{\circ} \mathrm{C}$ & .02 & -.01 \\
\hline .36 & $6 / 08 / 90$ & 0500 & 35 & 1160.4 & $34^{\circ} 05.5^{\prime} \mathrm{N}$ & $64^{\circ} 29.1^{\prime} \mathrm{W}$ & 36.69 & $23.5^{\circ} \mathrm{C}$ & .04 & .35 \\
\hline 37 & $6 / 08 / 90$ & 0807 & 36 & 1180.5 & $34^{\circ} 25.6^{\prime} \mathrm{N}$ & $64^{\circ} 27.8^{\prime} \mathrm{W}$ & 36.72 & $21.6^{\circ} \mathrm{C}$ & .04 & .06 \\
\hline
\end{tabular}


SS\# Date Time BT\# $\log (\mathrm{nm})$ Latitude $\quad$ Longitude $\%$ Sal. $\mathrm{T}_{\mathrm{s}} \quad$ Chl. $\mathrm{PO}_{4}$

\begin{tabular}{|c|c|c|c|c|c|c|c|c|c|}
\hline $6 / 08 / 90$ & 1300 & - & 1201.1 & $34^{\circ} 44.6^{\prime} \mathrm{N}$ & $64^{\circ} 33.1^{\prime} \mathrm{W}$ & 36.37 & $21.4^{\circ} \mathrm{C}$ & .05 & .18 \\
\hline 6/08/90 & 2245 & 37 & 1235.9 & $35^{\circ} 19.0^{\prime} \mathrm{N}$ & $64^{\circ} 46.0^{\prime} \mathrm{W}$ & 36.57 & $24.0^{\circ} \mathrm{C}$ & .09 & .02 \\
\hline $6 / 09 / 90$ & 2051 & 38 & 1278.6 & $36^{\circ} 03.9^{\prime} \mathrm{N}$ & $64^{\circ} 46.4^{\prime} \mathrm{W}$ & 36.56 & $24.3^{\circ} \mathrm{C}$ & .05 & .02 \\
\hline $6 / 10 / 90$ & 0715 & 39 & 1331.2 & $37^{\circ} 02.1^{\prime} \mathrm{N}$ & $64^{\circ} 41.3^{\prime} \mathrm{W}$ & 36.33 & $23.5^{\circ} \mathrm{C}$ & .06 & .17 \\
\hline $6 / 10 / 90$ & 1143 & 40 & 1353.2 & $37^{\circ} 22.9^{\prime} \mathrm{N}$ & $64^{\circ} 45.2^{\prime} \mathrm{W}$ & 36.70 & $23.9^{\circ} \mathrm{C}$ & .08 & .09 \\
\hline $6 / 10 / 90$ & 1606 & 41 & 1378.8 & $37^{\circ} 48.9^{\prime} \mathrm{N}$ & $64^{\circ} 38.9^{\prime} \mathrm{W}$ & 36.51 & $23.8^{\circ} \mathrm{C}$ & .05 & .39 \\
\hline $6 / 11 / 90$ & 0417 & 42 & 1399.9 & $38^{\circ} 08.4^{\prime} \mathrm{N}$ & $64^{\circ} 20.1^{\prime} \mathrm{W}$ & 36.47 & $25.3^{\circ} \mathrm{C}$ & .00 & -- \\
\hline $6 / 11 / 90$ & 0725 & 43 & 1419.7 & $38^{\circ} 22.0^{\prime} \mathrm{N}$ & $64^{\circ} 16.0^{\prime} \mathrm{W}$ & 36.32 & $25.0^{\circ} \mathrm{C}$ & .09 & .16 \\
\hline $6 / 11 / 90$ & 1752 & 44 & 1441.1 & $38^{\circ} 29.4^{\prime} \mathrm{N}$ & $63^{\circ} 44.2^{\prime} \mathrm{W}$ & 35.23 & $21.3^{\circ} \mathrm{C}$ & .17 & .13 \\
\hline $6 / 14 / 90$ & 0743 & 45 & 1606.6 & $39^{\circ} 20.7^{\prime} \mathrm{N}$ & $65^{\circ} 09.2 \mathrm{~W}$ & 35.69 & $19.0^{\circ} \mathrm{C}$ & .39 & .04 \\
\hline $6 / 14 / 90$ & 2300 & 46 & 1662.5 & $40^{\circ} 10.1^{\prime} \mathrm{N}$ & $65^{\circ} 05.4^{\circ} \mathrm{W}$ & 35.83 & $20.2^{\circ} \mathrm{C}$ & .12 & .05 \\
\hline $6 / 15 / 90$ & 0251 & 47 & 1681.6 & $40^{\circ} 28.6^{\prime} \mathrm{N}$ & $64^{\circ} 56.1^{\prime} \mathrm{W}$ & 34.96 & $17.3^{\circ} \mathrm{C}$ & .21 & .25 \\
\hline $6 / 15 / 90$ & 0348 & 48 & 1701.1 & $40^{\circ} 46.3^{\prime} \mathrm{N}$ & $64^{\circ} 54.5^{\prime} \mathrm{W}$ & 35.66 & $19.2^{\circ} \mathrm{C}$ & -- & .21 \\
\hline $6 / 15 / 90$ & 0925 & 49 & 1720.1 & $41^{\circ} 05.9^{\prime} \mathrm{N}$ & $64^{\circ} 48.4^{\prime} \mathrm{W}$ & 35.21. & $17.1^{\circ} \mathrm{C}$ & .26 & .11 \\
\hline $6 / 15 / 90$ & 1500 & 50 & 1738.8 & $41^{\circ} 28.5^{\prime} \mathrm{N}$ & $64^{\circ} 43.7^{\prime} \mathrm{W}$ & 35.42 & $15.9^{\circ} \mathrm{C}$ & .45 & -- \\
\hline $6 / 15 / 90$ & 1855 & 51 & 1756.0 & $41^{\circ} 47.4^{\prime} \mathrm{N}$ & $64^{\circ} 35.3^{\prime} \mathrm{W}$ & 33.62 & $13.4^{\circ} \mathrm{C}$ & .53 & .53 \\
\hline $6 / 15 / 90$ & 2000 & 52 & 1761.0 & $41^{\circ} 52.6^{\prime} \mathrm{N}$ & $64^{\circ} 32.9^{\prime} \mathrm{W}$ & - & $11.1^{\circ} \mathrm{C}$ & .47 & .51 \\
\hline$/ 15 / 90$ & 2205 & 53 & 1770.8 & $42^{\circ} 01.6 ’ \mathrm{~N}$ & $64^{\circ} 28.5^{\prime} \mathrm{W}$ & 32.07 & $10.5^{\circ} \mathrm{C}$ & .29 & .22 \\
\hline $6 / 16 / 90$ & 0450 & 54 & 1800.4 & $42^{\circ} 31.9^{\prime} \mathrm{N}$ & $64^{\circ} 19.5^{\prime} \mathrm{W}$ & 32.02 & $10.5^{\circ} \mathrm{C}$ & .30 & .39 \\
\hline $6 / 17 / 90$ & 0000 & 55 & 1821.7 & $42^{\circ} 56.4^{\prime} \mathrm{N}$ & $64^{\circ} 18.5^{\prime} \mathrm{W}$ & 31.68 & $10.5^{\circ} \mathrm{C}$ & 18 & .77 \\
\hline $6 / 18 / 90$ & 0350 & 56 & 1858.1 & $43^{\circ} 32.2^{\prime} \mathrm{N}$ & $64^{\circ} 04.4^{\prime} \mathrm{W}$ & 31.65 & $11.1^{\circ} \mathrm{C}$ & .27 & .14 \\
\hline $6 / 18 / 90$ & 0908 & 57 & 1880.1 & $43^{\circ} 54.2^{\prime} \mathrm{N}$ & $64^{\circ} 08.7^{\circ} \mathrm{W}$ & 31.20 & $9.4^{\circ} \mathrm{C}$ & .26 & .54 \\
\hline $6 / 22 / 90$ & 0310 & 58 & 1942.7 & $43^{\circ} 50.7^{\prime} \mathrm{N}$ & $64^{\circ} 26.0^{\prime} \mathrm{W}$ & 30.61 & $13.1^{\circ} \mathrm{C}$ & .12 & .45 \\
\hline $22 / 90$ & 0729 & 59 & 1961.0 & $43^{\circ} 32.3^{\prime} \mathrm{N}$ & $64^{\circ} 31.3^{\prime} \mathrm{W}$ & 31.15 & $11.7^{\circ} \mathrm{C}$ & .07 & .50 \\
\hline $6 / 22 / 90$ & 1231 & 60 & 1980.8 & $43^{\circ} 09.2^{\prime} \mathrm{N}$ & $64^{\circ} 31.1^{\prime} \mathrm{W}$ & 31.59 & $12.4^{\circ} \mathrm{C}$ & .09 & .37 \\
\hline $6 / 22 / 90$ & 1920 & 61 & 2000.0 & $43^{18.9^{\prime} \mathrm{N}}$ & $64^{\circ} 56.2^{\prime} \mathrm{W}$ & 31.70 & $11.6^{\circ} \mathrm{C}$ & .11 & .62 \\
\hline $6 / 23 / 90$ & 1115 & 62 & 2022.4 & $43^{\circ} 03.3^{\circ} \mathrm{N}$ & $65^{\circ} 16.9^{\prime} \mathrm{W}$ & 31.69 & $10.8^{\circ} \mathrm{C}$ & .20 & .79 \\
\hline $6 / 23 / 90$ & 1540 & 63 & 2059.6 & $42^{\circ} 43.0^{\prime} \mathrm{N}$ & $65^{\circ} 55.8^{\prime} \mathrm{W}$ & 32.81 & $8.8^{\circ} \mathrm{C}$ & .21 & .55 \\
\hline $6 / 23 / 90$ & 2130 & 65 & 2079.1 & $42^{\circ} 39.9^{\prime} \mathrm{N}$ & $66^{\circ} 27.7^{\top} \mathrm{W}$ & 31.93 & $9.8^{\circ} \mathrm{C}$ & .18 & 1.25 \\
\hline $6 / 24 / 90$ & 0035 & 66 & 2102.0 & $42^{\circ} 39.9^{\prime} \mathrm{N}$ & $67^{\circ} 00.8^{\prime} \mathrm{W}$ & 32.90 & $12.0^{\circ} \mathrm{C}$ & .32 & .55 \\
\hline $6 / 24 / 90$ & 0350 & 67 & 2123.1 & $42^{\circ} 38.3^{\prime} \mathrm{N}$ & $67^{\circ} 25.6^{\prime} \mathrm{W}$ & 32.85 & $12.0^{\circ} \mathrm{C}$ & .24 & .41 \\
\hline $6 / 24 / 90$ & 0800 & 68 & 2141.4 & $42^{\circ} 38.5^{\prime} \mathrm{N}$ & $67^{\circ} 48.0^{\prime} \mathrm{W}$ & 33.01 & $12.9^{\circ} \mathrm{C}$ & .27 & -- \\
\hline $6 / 24 / 90$ & 1250 & 69 & 2161.5 & $42^{\circ} 39.7^{\prime} \mathrm{N}$ & $68^{\circ} 18.8^{\circ} \mathrm{W}$ & 32.91 & $13.4^{\circ} \mathrm{C}$ & .31 & .20 \\
\hline $6 / 24 / 90$ & 1847 & 70 & 2181.9 & $42^{\circ} 41.0^{\prime} \mathrm{N}$ & $68^{\circ} 48.0^{\prime} \mathrm{W}$ & 32.55 & $12.7^{\circ} \mathrm{C}$ & .34 & -.01 \\
\hline $6 / 24 / 90$ & 2215 & 71 & 2205.4 & $42^{\circ} 44.8^{\prime} \mathrm{N}$ & $69^{\circ} 19.6^{\prime} \mathrm{W}$ & 32.92 & $12.9^{\circ} \mathrm{C}$ & .35 & .12 \\
\hline $6 / 25 / 90$ & 0220 & 72 & 2220.2 & $42^{\circ} 51.1 ' \mathrm{~N}$ & $69^{\circ} 35.4^{\prime} \mathrm{W}$ & 32.02 & $13.7^{\circ} \mathrm{C}$ & .30 & .34 \\
\hline $6 / 25 / 90$ & 0745 & 73 & 2239.3 & $4256.4^{\prime} \mathrm{N}$ & $70^{\circ} 00.6^{\prime} \mathrm{W}$ & 31.68 & $13.1^{\circ} \mathrm{C}$ & .45 & 1.17 \\
\hline $6 / 25 / 90$ & 1320 & 74 & 2261.2 & $42^{\circ} 52.8^{\prime} \mathrm{N}$ & $70^{\circ} 08.9^{\prime} \mathrm{W}$ & 31.67 & $13.7^{\circ} \mathrm{C}$ & .67 & -- \\
\hline $6 / 26 / 90$ & 0030 & 75 & 2283.1 & $42^{\circ} 37.7 ’ \mathrm{~N}$ & $3.4^{\prime} \mathrm{W}$ & -- & $14.8^{\circ} \mathrm{C}$ & .21 & .14 \\
\hline $6 / 26 / 90$ & 0350 & 76 & 2299.9 & $4239.8^{\prime} \mathrm{N}$ & $70^{\circ} 03.2^{\prime} \mathrm{W}$ & 31.75 & $13.3^{\circ} \mathrm{C}$ & .66 & -- \\
\hline $6 / 26 / 90$ & 1145 & 77 & 2322.2 & $42^{\circ} 35.9^{\prime} \mathrm{N}$ & $7.9^{\prime} \mathrm{W}$ & 31.79 & $16.6^{\circ} \mathrm{C}$ & .15 & .30 \\
\hline
\end{tabular}




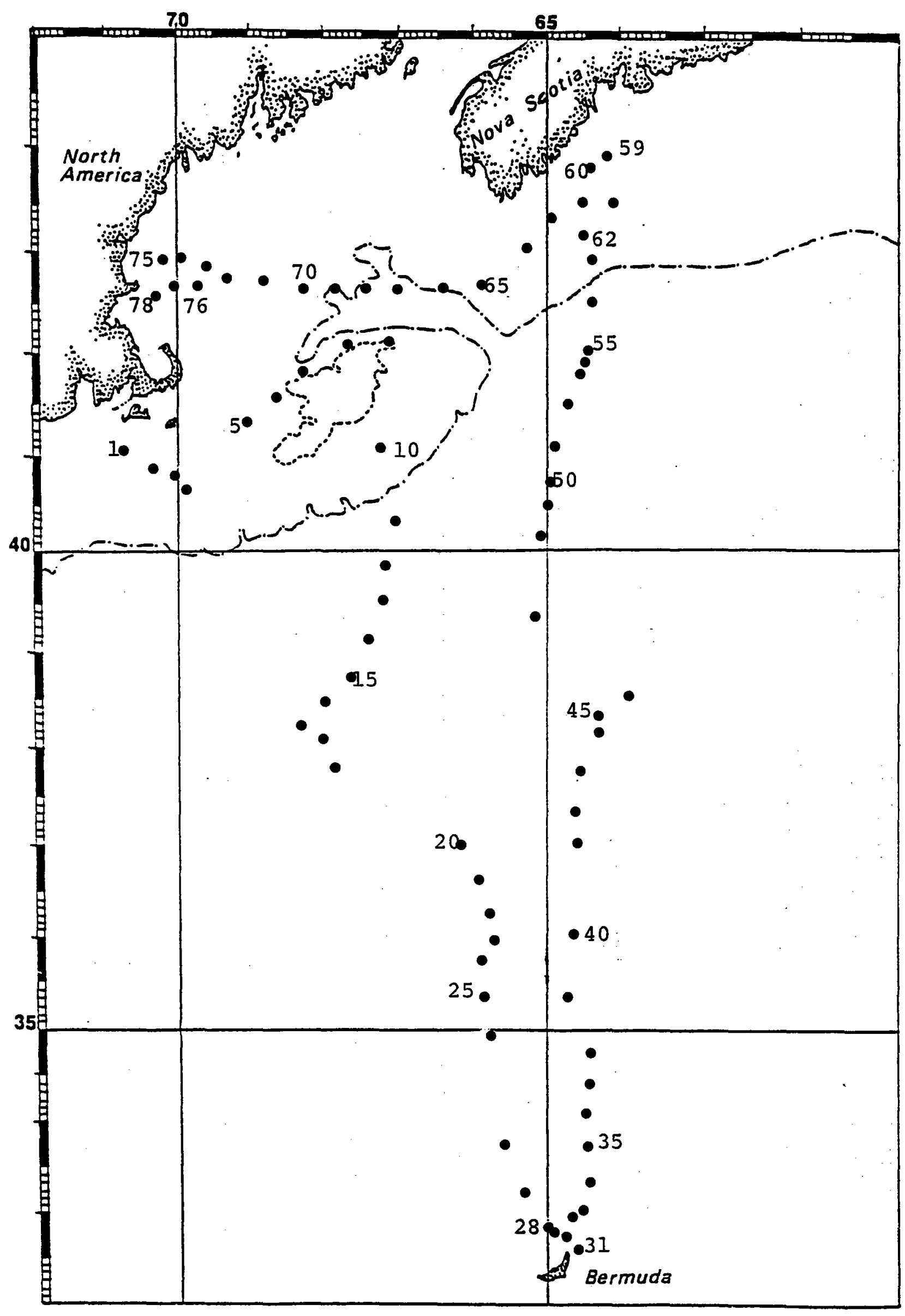

Figure 2 Surface Stations 
Table VI. Hydrostations and Operations for SSV Corwith Cramer Cruise C-112.

HC\# DATE TIME LOG (nm) LATITUDE LONGITUDE CTD BC MT NT SG OT *

\begin{tabular}{|c|c|c|c|c|c|c|c|c|c|c|}
\hline $5 / 25 / 90$ & 0212 & 66.9 & $40^{\circ} 40.1^{\prime} \mathrm{N}$ & $69^{\circ} 55.0^{\prime} \mathrm{W}$ & $\mathrm{X}$ & $\mathrm{X}$ & $\mathrm{X}$ & $\mathbf{X}$ & & \\
\hline $5 / 25 / 90$ & 1505 & 139.8 & $41^{\circ} 22.6^{\prime} \mathrm{N}$ & $68^{\circ} 56.3^{\prime} \mathrm{W}$ & $\mathbf{X}$ & $\mathrm{X}$ & $\mathrm{X}$ & $\mathrm{X}$ & & \\
\hline $5 / 26 / 90$ & 0200 & 201.5 & $42^{\circ} 01.3^{\prime} \mathrm{N}$ & $67^{\circ} 56.4^{\prime} \mathrm{W}$ & $\mathrm{X}$ & $\mathrm{X}$ & $\mathrm{X}$ & $\mathrm{X}$ & & \\
\hline $5 / 26 / 90$ & 1105 & 247.5 & $42^{\circ} 15.0^{\prime} \mathrm{N}$ & $67^{\circ} 13.0^{\prime} \mathrm{W}$ & $\mathrm{X}$ & $\mathrm{X}$ & $\mathrm{X}$ & $\mathrm{X}$ & & \\
\hline $5 / 26 / 90$ & 1348 & 253.5 & $42^{\circ} 09.8 \mathrm{~N}$ & $67^{\circ} 11.4^{\prime} \mathrm{W}$ & $\mathrm{X}$ & $\mathrm{X}$ & $X$ & & & \\
\hline $5 / 26 / 90$ & 1510 & 255.9 & $42^{\circ} 06.5^{\circ} \mathrm{N}$ & $67^{\circ} 08.0^{\prime} \mathrm{W}$ & $\mathrm{X}$ & $\mathrm{X}$ & $\mathrm{X}$ & & & \\
\hline $5 / 26 / 90$ & 1620 & 258.2 & $42^{\circ} 03.1^{\prime} \mathrm{N}$ & $67^{\circ} 08.9^{\prime} \mathrm{W}$ & $\mathrm{X}$ & $\mathrm{X}$ & $\mathrm{X}$ & & & \\
\hline $5 / 26 / 90$ & 1900 & 262.3 & $41^{\circ} 58.4^{\prime} \mathrm{N}$ & $67^{\circ} 11.5^{\prime} \mathrm{W}$ & $\mathrm{X}$ & $\mathrm{X}$ & & $\mathrm{X}$ & & \\
\hline $5 / 26 / 90$ & 2240 & 272.3 & $41^{\circ} 57.4^{\prime} \mathrm{N}$ & $67^{\circ} 10.6^{\prime} \mathrm{W}$ & $\mathrm{X}$ & $\mathrm{X}$ & $\mathrm{X}$ & $\mathrm{x}$ & & \\
\hline $5 / 27 / 90$ & 0640 & 301.8 & $41^{\circ} 26.6^{\prime} \mathrm{N}$ & $67^{\circ} 13.2^{\prime} \mathrm{W}$ & $\mathrm{X}$ & $\mathrm{X}$ & $\mathrm{X}$ & & & \\
\hline $5 / 27 / 90$ & 1340 & 304.0 & $41^{\circ} 28.0^{\prime} \mathrm{N}$ & $67^{\circ} 20.8^{\prime} \mathrm{W}$ & & & $X$ & & & \\
\hline $5 / 27 / 90$ & 1425 & 305.5 & $41^{\circ} 22.0^{\prime} \mathrm{N}$ & $67^{\circ} 18.0^{\prime} \mathrm{W}$ & & & & & $\mathrm{X}$ & $\mathbf{X}$ \\
\hline $5 / 27 / 90$ & 2113 & 319.8 & $41^{\circ} 22.8^{\prime} \mathrm{N}$ & $67^{\circ} 20.8^{\prime} \mathrm{W}$ & $\mathbf{X}$ & & $\mathrm{X}$ & & & \\
\hline $5 / 28 / 90$ & 0623 & 357.8 & $40^{\circ} 45.0^{\prime} \mathrm{N}$ & $67^{\circ} 08.2^{\prime} \mathrm{W}$ & & & & & $\mathrm{X}$ & $\mathrm{X}$ \\
\hline $5 / 28 / 90$ & 1138 & 376.2 & $40^{\circ} 27.4^{\prime} \mathrm{N}$ & $67^{\circ} 04.4^{\prime} \mathrm{W}$ & & & & $\mathrm{X}$ & & \\
\hline $5 / 28 / 90$ & 1948 & 432.5 & $39^{\circ} 42.4^{\prime} \mathrm{N}$ & $67^{\circ} 14.3^{\prime} \mathrm{W}$ & & & $\mathrm{X}$ & & & \\
\hline $5 / 28 / 90$ & 0000 & 455.0 & $39^{\circ} 20.0^{\prime} \mathrm{N}$ & $67^{\circ} 22.4^{\prime} \mathrm{W}$ & $\mathrm{X}$ & & $\mathrm{X}$ & $\mathrm{X}$ & & \\
\hline $5 / 29 / 90$ & 0829 & 464.9 & $39^{\circ} 04.1^{\prime} \mathrm{N}$ & $67^{\circ} 34.1^{\prime} \mathrm{W}$ & $\mathrm{X}$ & & & & & \\
\hline $5 / 30 / 90$ & 0010 & 548.1 & $38^{\circ} 14.1^{\prime} \mathrm{N}$ & $68^{\circ} 23.5^{\prime} \mathrm{W}$ & & & & $\mathrm{X}$ & & \\
\hline $5 / 30 / 90$ & 0745 & 591.2 & $37^{\circ} 48.6^{\prime} \mathrm{N}$ & $67^{\circ} 40.4^{\prime} \mathrm{W}$ & $\mathrm{X}$ & & & & & \\
\hline $5 / 31 / 90$ & 1202 & 732.4 & $36^{\circ} 45.3^{\prime} \mathrm{N}$ & $66^{\circ} 01.1^{\prime} \mathrm{W}$ & $\mathrm{X}$ & & $\mathrm{X}$ & $\mathrm{X}$ & & \\
\hline $5 / 31 / 90$ & 2324 & 781.1 & $35^{\circ} 57.8^{\prime} \mathrm{N}$ & $65^{\circ} 45.8^{\prime} \mathrm{W}$ & $\mathrm{X}$ & & $\mathrm{X}$ & $\mathrm{X}$ & & \\
\hline $6 / 01 / 90$ & 1130 & 815.3 & $35^{\circ} 27.8^{\prime} \mathrm{N}$ & $65^{\circ} 54.0^{\prime} \mathrm{W}$ & $\mathrm{X}$ & $\mathrm{X}$ & & & & \\
\hline $6 / 01 / 90$ & 2359 & 851.0 & $34^{\circ} 57.1^{\prime} \mathrm{N}$ & $65^{\circ} 47.9^{\prime} \mathrm{W}$ & $\mathrm{X}$ & & & $\mathrm{X}$ & & \\
\hline $6 / 02 / 90$ & 2335 & 938.9 & $33^{\circ} 30.0^{\prime} \mathrm{N}$ & $65^{\circ} 31.5^{\supset} \mathrm{W}$ & $X$ & & & $\mathrm{X}$ & & \\
\hline $6 / 03 / 90$ & 1655 & 1023.3 & $32^{\circ} 56.4^{\prime} \mathrm{N}$ & $64^{\circ} 43.6^{\prime} \mathrm{W}$ & $\mathrm{X}$ & & $\mathrm{X}$ & $\mathrm{X}$ & & \\
\hline $6 / 04 / 90$ & 0530 & 1059.9 & $32^{\circ} 26.6^{\prime} \mathrm{N}$ & $64^{\circ} 33.4^{\prime} \mathrm{W}$ & & & & & $\mathrm{X}$ & \\
\hline $6 / 08 / 90$ & 1205 & 1201.1 & $34^{\circ} 44.6^{\prime} \mathrm{N}$ & $64^{\circ} 33.1^{\prime} \mathrm{W}$ & $\mathrm{X}$ & & & $\mathrm{X}$ & & \\
\hline $6 / 08 / 90$ & 2319 & 1236.8 & $35^{\circ} 20.5^{\prime} \mathrm{N}$ & $64^{\circ} 48.1^{\prime} \mathrm{W}$ & & & $\mathrm{X}$ & $\mathrm{X}$ & & \\
\hline $6 / 09 / 90$ & 1558 & 1255.8 & $35^{\circ} 56.0^{\prime} \mathrm{N}$ & $64^{\circ} 49.4^{\prime} \mathrm{W}$ & & & & $\mathrm{X}$ & & \\
\hline $6 / 09 / 90$ & 1855 & 1261.1 & $35^{\circ} 55.9^{\prime} \mathrm{N}$ & $64^{\circ} 46.7^{\prime} \mathrm{W}$ & & & & $\mathrm{X}$ & & \\
\hline $6 / 09 / 90$ & 2315 & 1289.9 & $36^{\circ} 17.3^{\prime} \mathrm{N}$ & $64^{\circ} 48.2^{\prime} \mathrm{W}$ & $\mathrm{X}$ & & & & & \\
\hline $6 / 10 / 90$ & 1047 & 1348.7 & $37^{\circ} 18.6^{\prime} \mathrm{N}$ & $64^{\circ} 47.0^{\circ} \mathrm{W}$ & & & & $\mathrm{X}$ & & \\
\hline $6 / 10 / 90$ & 2030 & 1391.6 & $38^{\circ} 00.1^{\prime} \mathrm{N}$ & $64^{\circ} 28.4^{\prime} \mathrm{W}$ & $\mathrm{X}$ & $\mathrm{X}$ & & & & \\
\hline $6 / 11 / 90$ & 0915 & 1424.5 & $38^{\circ} 21.1^{\prime} \mathrm{N}$ & $63^{\circ} 58.0^{\circ} \mathrm{W}$ & $\mathrm{X}$ & $\mathrm{X}$ & & & & \\
\hline $6 / 11 / 90$ & 2015 & 1454.4 & $38^{\circ} 35.4^{\prime} \mathrm{N}$ & $63^{\circ} 58.4^{\prime} \mathrm{W}$ & $\mathrm{X}$ & $\mathrm{X}$ & & $\mathrm{X}$ & & \\
\hline $6 / 12 / 90$ & 0045 & 1459.9 & $38^{\circ} 40.2^{\prime} \mathrm{N}$ & $63^{\circ} 59.7 \mathrm{~W}$ & $\mathrm{X}$ & $\mathrm{X}$ & & & & \\
\hline $6 / 12 / 90$ & 1158 & 1488.5 & $38^{\circ} 50.1^{\prime} \mathrm{N}$ & $64^{\circ} 32.9^{\prime} \mathrm{W}$ & $\mathrm{X}$ & $\mathrm{X}$ & & & & \\
\hline $6 / 13 / 90$ & 2230 & 1587.6 & $39^{\circ} 10.0^{\prime} \mathrm{N}$ & $65^{\circ} 16.8^{\prime} \mathrm{W}$ & $\mathrm{X}$ & $\mathrm{X}$ & $\mathrm{X}$ & $\mathrm{X}$ & & \\
\hline $6 / 14 / 90$ & 1030 & 1619.6 & $39^{\circ} 31.0^{\prime} \mathrm{N}$ & $65^{\circ} 04.6^{\prime} \mathrm{W}$ & $\mathrm{X}$ & $\mathrm{X}$ & & & & \\
\hline $6 / 14 / 90$ & 2345 & 1664.8 & $40^{\circ} 13.7^{\prime} \mathrm{N}$ & $65^{\circ} 04.7^{\prime} \mathrm{W}$ & & & & $\mathrm{X}$ & & \\
\hline
\end{tabular}


HC\# DATE TIME LOG (nm) LATITUDE LONGITUDE CTD BC MT NT SG OT

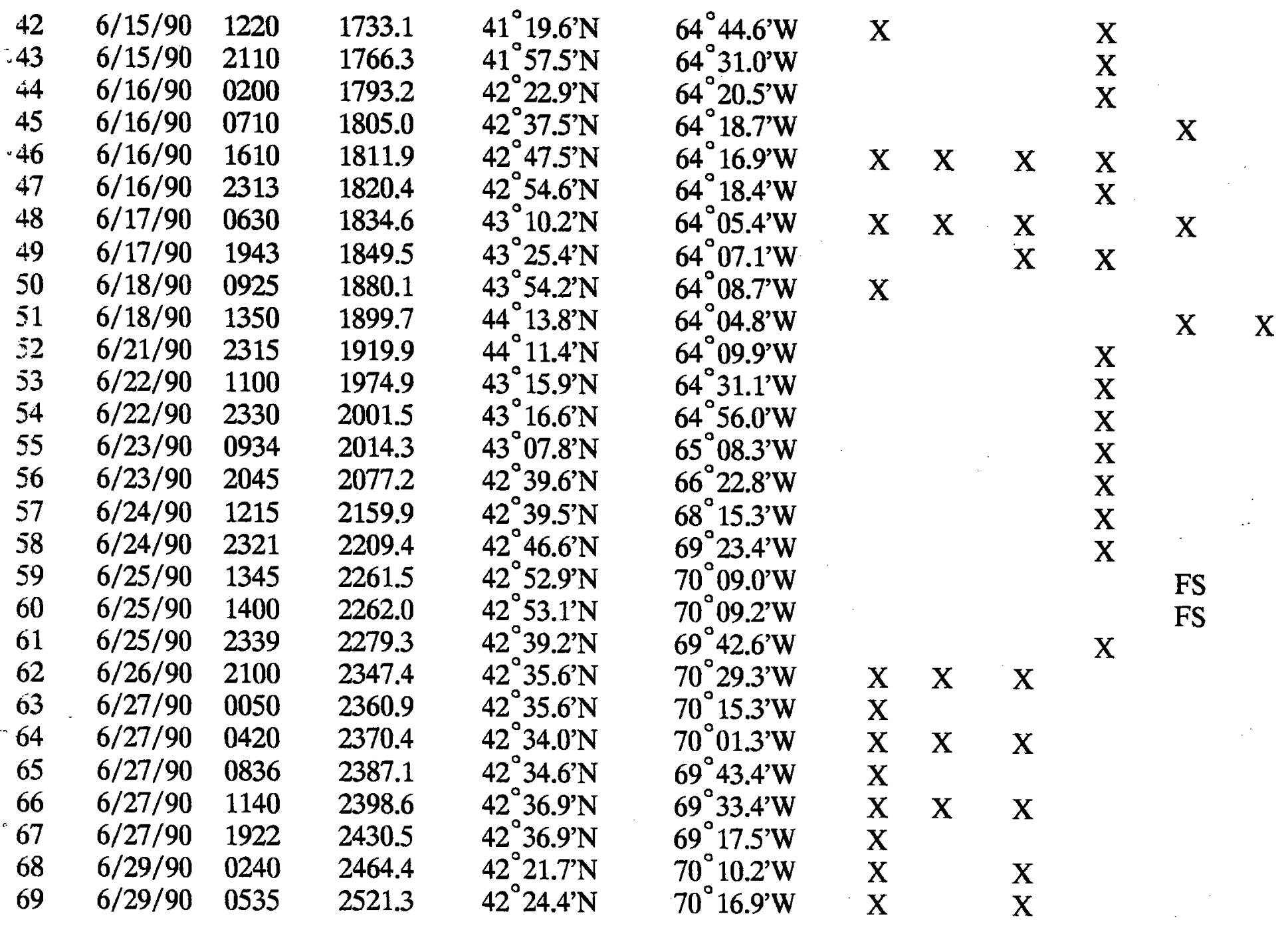

CTD = conductivity-temperature-depth profile

$\mathrm{BC}=$ Niskin bottle cast

$\mathrm{MT}=$ meter net tow

NT $=$ neuston net tow

$\mathrm{SG}=$ Shipek grab

$\mathrm{OT}=$ otter trawl 


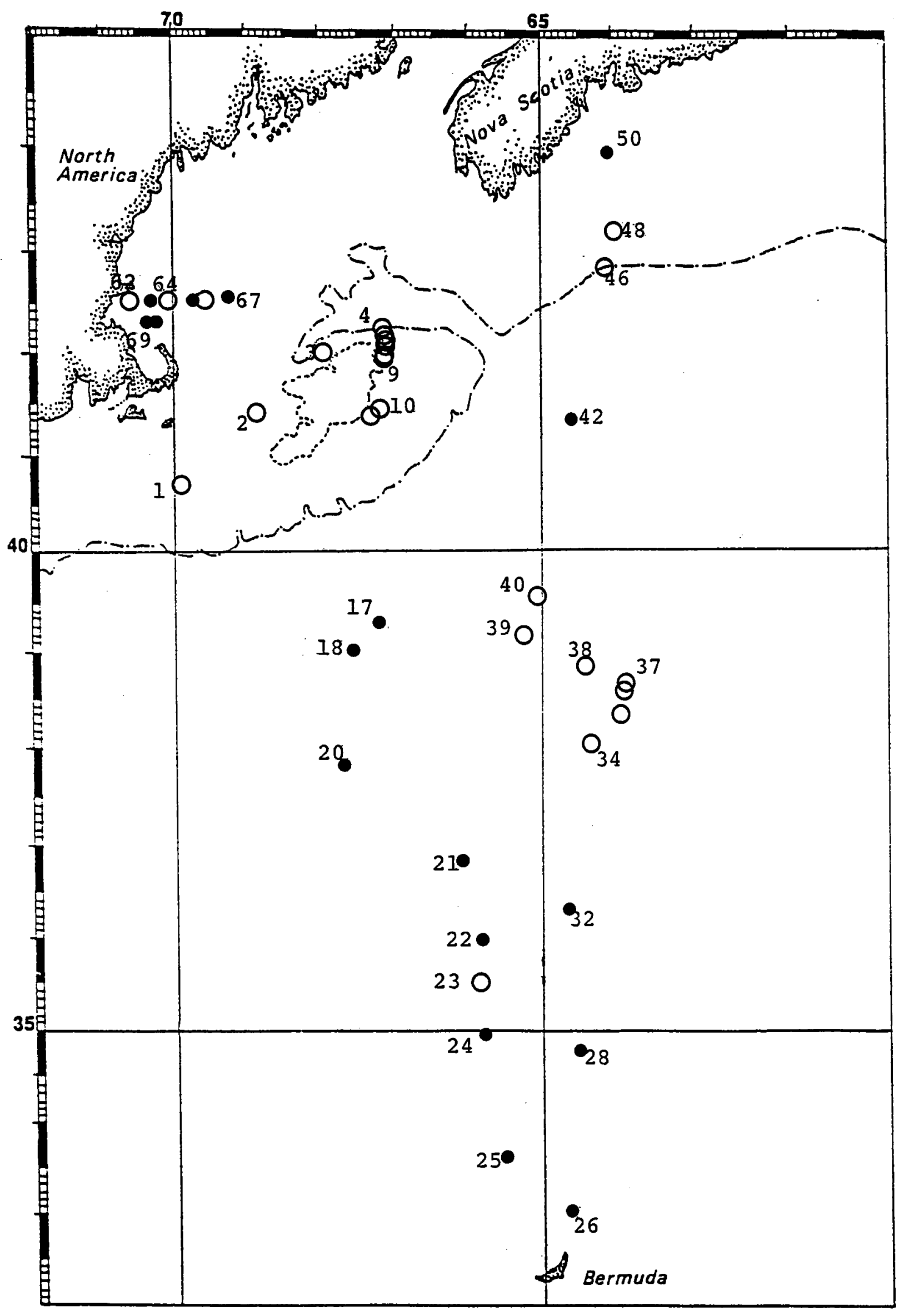

Figure 3 - CTD Stations O Hydrocast stations 
Table VII. CTD data for SSV Corwith Cramer Cruise C-112 (stations $2,4,5,6, \overline{7,8,9}, 1 \overline{0,23,34}, 35,36,37,38$, 39,40 , and 48 ).






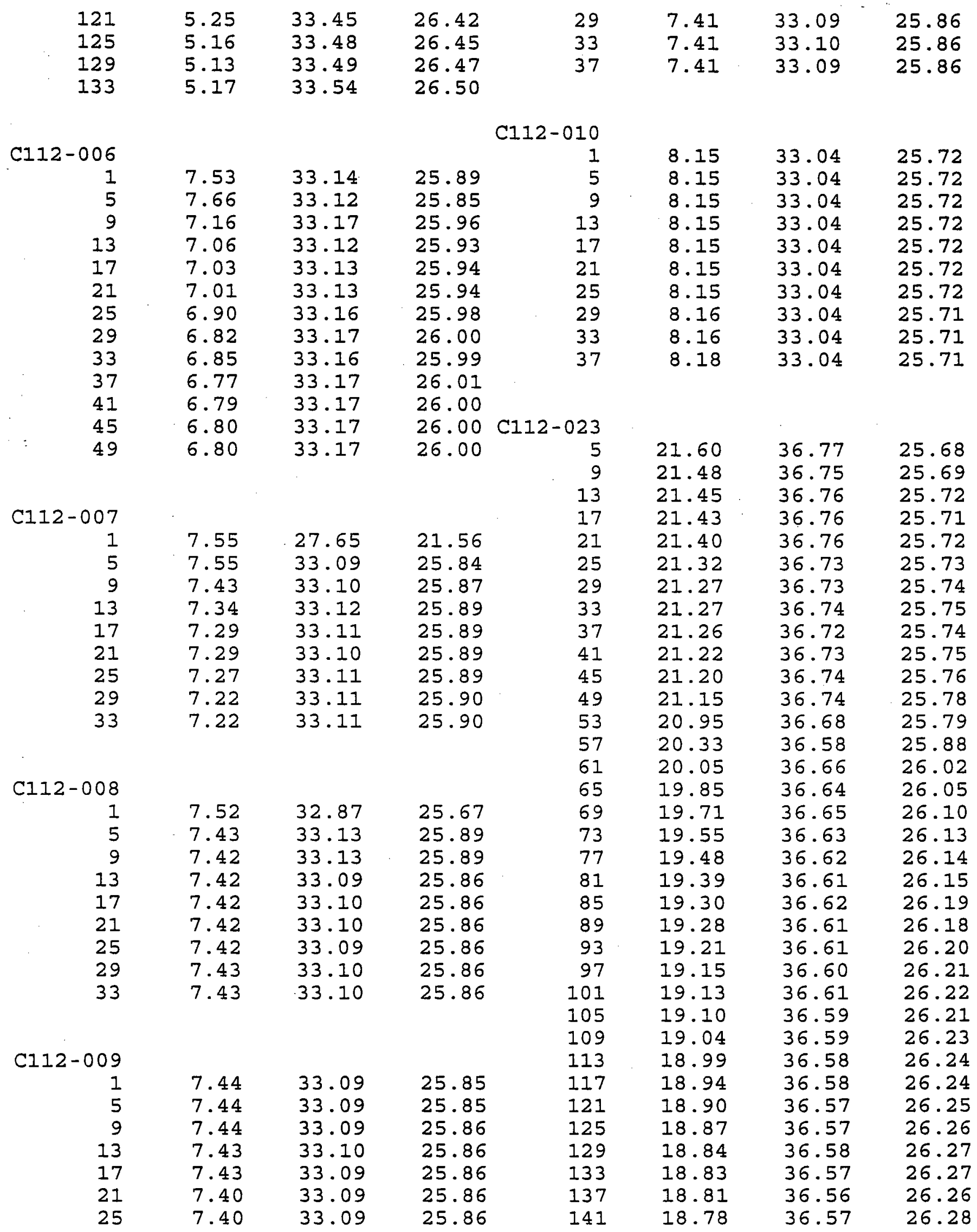




\begin{tabular}{|c|c|c|c|c|c|c|c|}
\hline 145 & 18.77 & 36.56 & 26.28 & 701 & 13.41 & 35.72 & 26.87 \\
\hline 149 & 18.76 & 36.57 & 26.28 & 721 & 13.02 & 35.65 & 26.89 \\
\hline 153 & 18.75 & 36.57 & 26.29 & 741 & 12.57 & 35.60 & 26.95 \\
\hline 157 & 18.75 & 36.57 & 26.29 & 761 & 12.18 & 35.54 & 26.97 \\
\hline 161 & 18.72 & 36.57 & 26.29 & 781 & 11.79 & 35.48 & 27.00 \\
\hline 165 & 18.72 & 36.57 & 26.29 & 801 & 11.35 & 35.42 & 27.04 \\
\hline 169 & 18.68 & 36.56 & 26.30 & 821 & 10.57 & 35.33 & 27.11 \\
\hline 173 & 18.66 & 36.56 & 26.30 & 841 & 10.10 & 35.30 & 27.17 \\
\hline 177 & 18.65 & 36.56 & 26.30 & 861 & 9.86 & 35.25 & 27.17 \\
\hline 181 & 18.61 & 36.55 & 26.31 & 881 & 9.51 & 35.22 & 27.21 \\
\hline 185 & 18.60 & 36.55 & 26.31 & 901 & 9.07 & 35.17 & 27.24 \\
\hline 189 & 18.60 & 36.55 & 26.31 & 921 & 8.60 & 35.16 & 27.31 \\
\hline 193 & 18.59 & 36.56 & 26.32 & 941 & 8.24 & 35.10 & 27.31 \\
\hline 197 & 18.58 & 36.54 & 26.31 & 961 & 7.90 & 35.09 & 27.36 \\
\hline 201 & 18.55 & 36.54 & 26.32 & 981 & 7.57 & 35.11 & 27.42 \\
\hline 211 & 18.53 & 36.55 & 26.32 & 1001 & 7.33 & 35.07 & 27.43 \\
\hline 221 & 18.50 & 36.54 & 26.33 & 1021 & 7.04 & 35.07 & 27.47 \\
\hline 231 & 18.48 & 36.54 & 26.33 & 1041 & 6.74 & 35.08 & 27.52 \\
\hline 241 & 18.47 & 36.54 & 26.34 & 1061 & 6.50 & 35.07 & 27.55 \\
\hline 251 & 18.44 & 36.54 & 26.34 & 1081 & 6.28 & 35.06 & 27.56 \\
\hline 261 & 18.41 & 36.54 & 26.35 & 1101 & 6.13 & 35.04 & 27.57 \\
\hline 271 & 18.34 & 36.52 & 26.35 & 1121 & 5.98 & 35.04 & 27.59 \\
\hline 281 & 18.29 & 36.52 & 26.36 & 1141 & 5.87 & 35.05 & 27.61 \\
\hline 291 & 18.20 & 36.51 & 26.38 & 1161 & 5.72 & 35.04 & 27.62 \\
\hline 301 & 18.15 & 36.50 & 26.38 & 1181 & 5.58 & 35.03 & 27.63 \\
\hline 311 & 18.09 & 36.49 & 26.39 & 1201 & 5.42 & 35.03 & 27.65 \\
\hline 321 & 18.05 & 36.47 & 26.39 & 1221 & 5.29 & 35.02 & 27.66 \\
\hline 331 & 17.99 & 36.47 & 26.40 & 1241 & 5.16 & 35.02 & 27.67 \\
\hline 341 & 17.94 & 36.47 & 26.42 & 1261 & 5.08 & 35.02 & 27.68 \\
\hline 351 & 17.91 & 36.46 & 26.42 & 1281 & 5.00 & 35.01 & 27.69 \\
\hline 361 & 17.83 & 36.45 & 26.42 & 1301 & 4.94 & 35.01 & 27.69 \\
\hline 371 & 17.79 & 36.45 & 26.43 & 1321 & 4.86 & 35.01 & 27.70 \\
\hline 381 & 17.71 & 36.43 & 26.44 & 1341 & 4.79 & 35.00 & 27.70 \\
\hline 391 & 17.64 & 36.42 & 26.45 & 1361 & 4.73 & 35.00 & 27.70 \\
\hline 401 & 17.59 & 36.42 & 26.46 & 1381 & 4.67 & 35.00 & 27.71 \\
\hline 411 & 17.52 & 36.41 & 26.47 & 1401 & 4.64 & 34.99 & 27.71 \\
\hline 421 & 17.43 & 36.39 & 26.48 & 1421 & 4.57 & 34.99 & 27.72 \\
\hline 431 & 17.34 & 36.38 & 26.49 & 1441 & 4.51 & 34.98 & 27.72 \\
\hline 441 & 17.23 & 36.36 & 26.50 & 1461 & 4.48 & 34.99 & 27.72 \\
\hline 451 & 17.14 & 36.34 & 26.51 & 1481 & 4.42 & 34.98 & 27.73 \\
\hline 461 & 17.07 & 36.33 & 26.52 & 1501 & 4.36 & 34.97 & 27.73 \\
\hline 471 & 16.97 & 36.32 & 26.54 & 1521 & 4.33 & 34.97 & 27.73 \\
\hline 481 & 16.84 & 36.30 & 26.55 & 1541 & 4.30 & 34.97 & 27.73 \\
\hline 491 & 16.67 & 36.28 & 26.58 & 1561 & 4.27 & 34.97 & 27.73 \\
\hline 501 & 16.50 & 36.23 & 26.58 & 1581 & 4.24 & 34.97 & 27.73 \\
\hline 521 & 16.20 & 36.18 & 26.61 & 1601 & 4.21 & 34.97 & 27.74 \\
\hline 541 & .15 .95 & 36.13 & 26.63 & 1621 & 4.19 & 34.96 & .27 .74 \\
\hline 561 & 15.61 & 36.08 & 26.67 & 1641 & 4.17 & 34.96 & 27.74 \\
\hline 581 & 15.40 & 36.04 & 26.68 & 1661 & 4.15 & 34.96 & 27.74 \\
\hline 601 & 15.23 & 36.01 & 26.70 & 1681 & 4.13 & 34.96 & 27.74 \\
\hline 621 & 15.00 & 35.98 & 26.72 & 1701 & 4.11 & 34.96 & 27.74 \\
\hline 641 & 14.76 & 35.88 & 26.71 & 1721 & 4.10 & 34.96 & 27.74 \\
\hline $66 I$ & 14.42 & 35.88 & 26.78 & 1741 & 4.08 & 34.96 & 27.75 \\
\hline 6 & 13.86 & 35.79 & 26.83 & 1761 & 4.05 & 34.96 & 27.75 \\
\hline
\end{tabular}




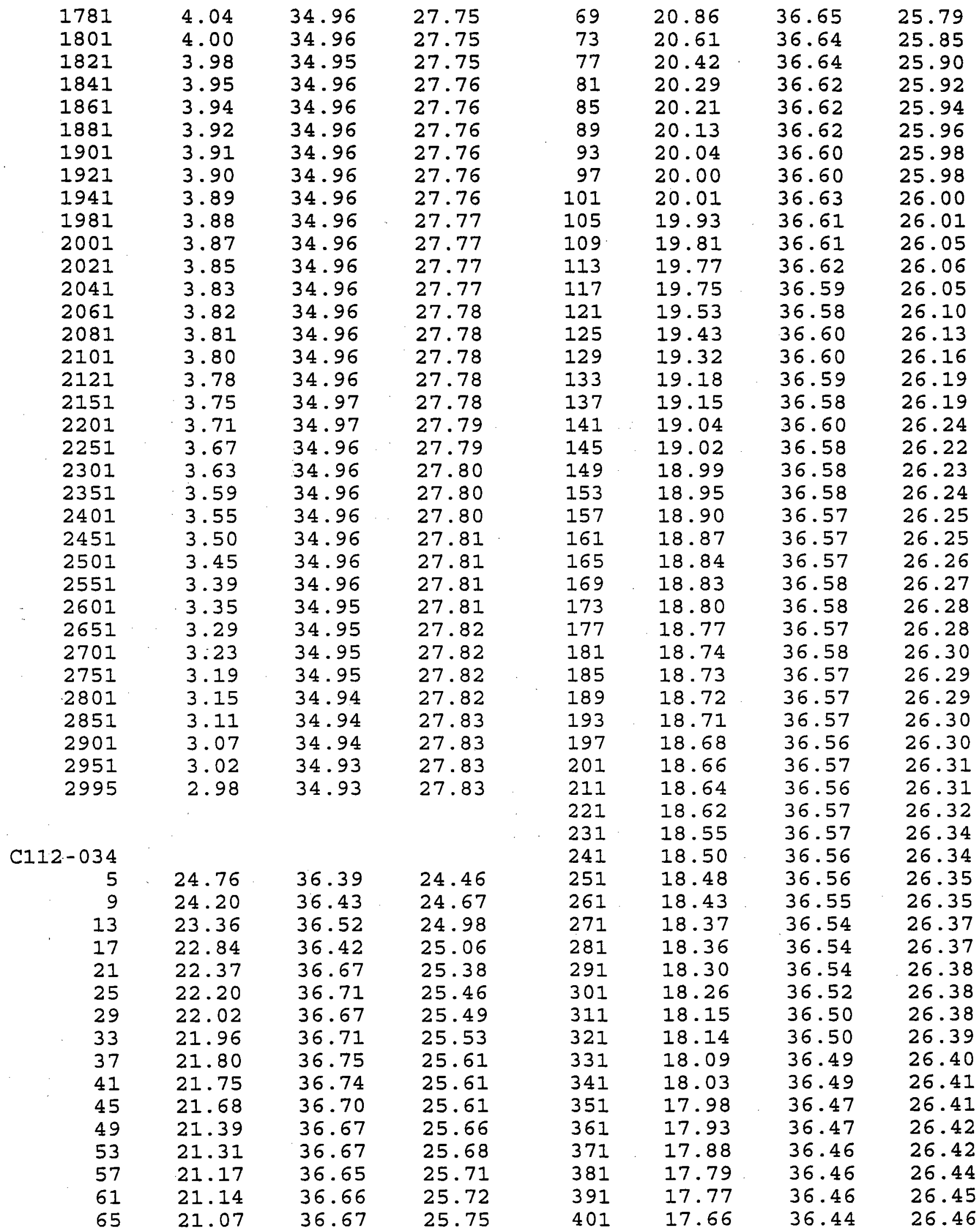




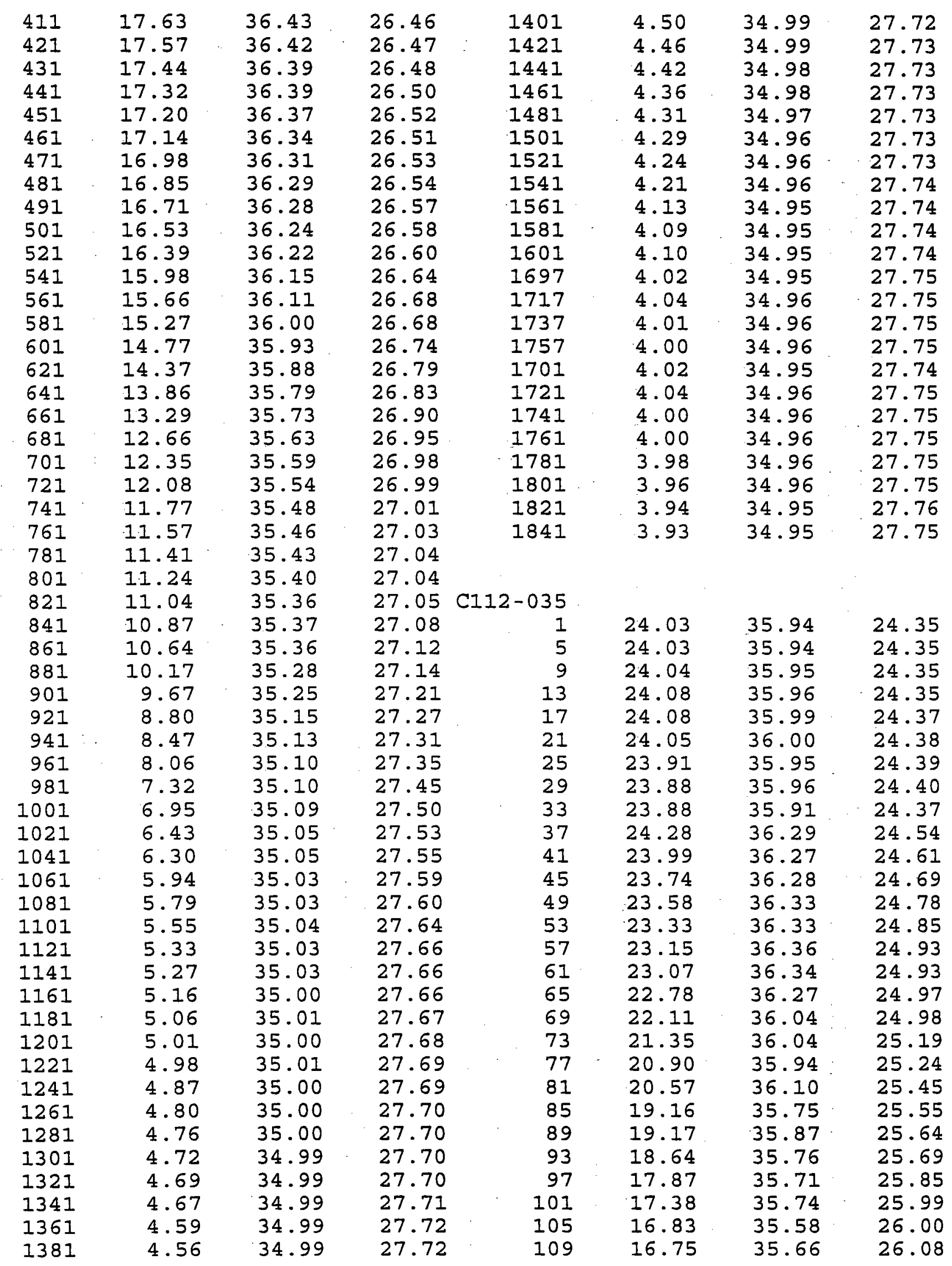




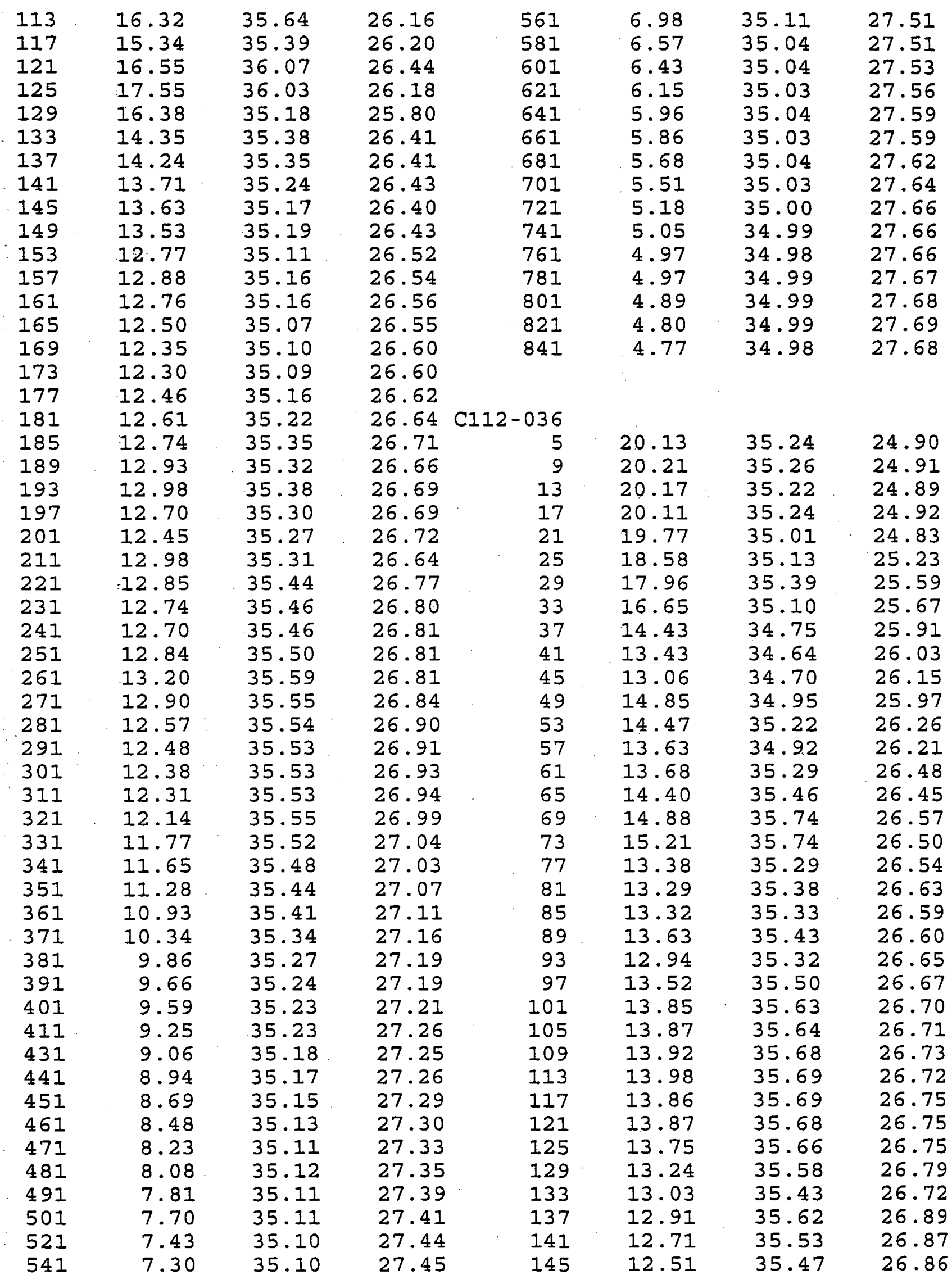




\begin{tabular}{|c|c|c|c|c|c|c|c|}
\hline & & & & & & & \\
\hline 149 & 12.39 & 35.43 & 26.85 & 721 & 5.00 & 35.01 & 27.68 \\
\hline 153 & 12.27 & 35.42 & 26.86 & 741 & 4.86 & 35.03 & 27.72 \\
\hline 157 & 12.21 & 35.44 & 26.89 & 761 & 4.68 & 34.97 & 27.69 \\
\hline 161 & 12.21 & 35.47 & 26.91 & 781 & 4.44 & 34.95 & 27.70 \\
\hline 165 & 12.28 & 35.48 & 26.91 & 801 & 4.50 & 34.96 & 27.70 \\
\hline 169 & 12.27 & 35.50 & 26.93 & 821 & 4.57 & 34.97 & 27.70 \\
\hline 173 & 12.22 & 35.52 & 26.95 & 841 & 4.57 & 34.98 & 27.71 \\
\hline 177 & 12.25 & 35.53 & 26.95 & 861 & 4.56 & 34.98 & 27.71 \\
\hline 181 & 12.13 & 35.50 & 26.95 & 881 & 4.53 & 34.98 & 27.71 \\
\hline 185 & 12.01 & 35.53 & 27.00 & 901 & 4.48 & 34.98 & 27.72 \\
\hline 189 & 12.00 & 35.52 & 26.99 & 921 & 4.42 & 34.97 & 27.72 \\
\hline 193 & 11.88 & 35.46 & 26.97 & 941 & 4.38 & 34.97 & 27.72 \\
\hline 197 & 11.78 & 35.44 & 26.98 & 961 & 4.37 & 34.97 & 27.72 \\
\hline 201 & 11.63 & 35.46 & 27.02 & 981 & 4.35 & 34.97 & 27.72 \\
\hline 211 & 11.27 & 35.46 & 27.08 & 1001 & 4.33 & 34.97 & 27.73 \\
\hline 221 & 11.09 & 35.34 & 27.03 & 1021 & 4.29 & 34.97 & 27.73 \\
\hline 231 & 10.79 & 35.37 & 27.10 & 1041 & 4.25 & 34.96 & 27.73 \\
\hline 241 & 10.72 & 35.36 & 27.11 & 1061 & 4.22 & 34.96 & 27.73 \\
\hline 251 & 10.47 & 35.32 & 27.12 & 1081 & 4.20 & 34.96 & 27.74 \\
\hline 261 & 10.19 & 35.26 & 27.12 & 1101 & 4.20 & 34.96 & 27.73 \\
\hline 271 & 9.77 & 35.25 & 27.19 & 1121 & 4.17 & 34.96 & 27.74 \\
\hline 281 & 9.67 & 35.23 & 27.19 & 1141 & 4.14 & 34.96 & 27.74 \\
\hline 291 & 9.52 & 35.22 & 27.21 & 1161 & 4.11 & 34.96 & 27.74 \\
\hline 301 & 9.29 & 35.18 & 27.21 & 1181 & 4.09 & 34.96 & 27.74 \\
\hline 311 & 9.16 & 35.18 & 27.23 & 1201 & 4.07 & 34.96 & 27.74 \\
\hline 321 & 8.96 & 35.16 & 27.25 & 1221 & 4.06 & 34.95 & 27.74 \\
\hline 331 & 8.77 & 35.16 & 27.28 & 1241 & 4.00 & 34.95 & 27.75 \\
\hline 341 & 8.54 & 35.15 & 27.31 & 1261 & 3.95 & 34.94 & 27.75 \\
\hline 351 & 8.45 & 35.12 & 27.30 & 1281 & 3.93 & .34 .94 & 27.75 \\
\hline 361 & 8.24 & 35.13 & 27.34 & 1301 & 3.89 & 34.94 & 27.75 \\
\hline 371 & 8.02 & 35.09 & 27.34 & 1321 & 3.85 & 34.93 & 27.75 \\
\hline 381 & 7.74 & 35.08 & 27.38 & 1341 & 3.80 & 34.93 & 27.75 \\
\hline 391 & 7.63 & 35.06 & 27.37 & 1361 & 3.77 & 34.92 & 27.75 \\
\hline 401 & 7.05 & 34.99 & 27.40 & 1381 & 3.75 & 34.92 & 27.75 \\
\hline 411 & 6.85 & 34.97 & 27.42 & 1401 & 3.72 & 34.92 & 27.75 \\
\hline 421 & 6.75 & 34.98 & 27.44 & 1421 & 3.69 & 34.91 & 27.75 \\
\hline 431 & 6.77 & 34.96 & 27.42 & 1441 & 3.68 & 34.91 & 27.75 \\
\hline 441 & 6.60 & 34.97 & 27.45 & 1461 & 3.65 & $34.9 I$ & 27.75 \\
\hline 451 & 6.49 & 35.00 & 27.49 & 1481 & 3.66 & 34.91 & 27.75 \\
\hline 461 & 6.67 & 35.02 & 27.48 & 1501 & 3.67 & 34.92 & 27.76 \\
\hline 471 & 6.55 & 35.04 & 27.51 & 1521 & 3.66 & 34.92 & 27.76 \\
\hline 481 & 6.45 & 35.03 & 27.52 & 1541 & 3.65 & 34.92 & 27.76 \\
\hline 491 & 6.37 & 35.04 & 27.54 & 1561 & 3.64 & 34.92 & 27.76 \\
\hline 501 & 6.15 & 35.00 & 27.54 & 1581 & 3.64 & 34.92 & 27.76 \\
\hline 521 & 6.10 & 35.03 & 27.56 & 1601 & 3.66 & 34.92 & 27.76 \\
\hline 541 & 5.99 & 35.04 & 27.58 & 1621 & 3.65 & 34.92 & 27.76 \\
\hline 561 & 5.90 & 35.04 & 27.59 & 1641 & 3.64 & 34.93 & 27.76 \\
\hline 581 & 5.71 & 35.03 & 27.62 & 1661 & 3.65 & 34.93 & 27.77 \\
\hline 601 & 5.61 & 35.03 & 27.62 & 1681 & 3.69 & 34.94 & 27.77 \\
\hline 621 & 5.47 & 35.02 & 27.64 & 1701 & 3.73 & 34.95 & 27.77 \\
\hline 641 & 5.27 & 35.01 & 27.65 & 1721 & 3.72 & 34.95 & 27.77 \\
\hline 661 & 5.21 & 35.01 & 27.66 & 1741 & 3.70 & 34.95 & 27.77 \\
\hline 681 & 5.10 & 35.00 & 27.66 & 1761 & 3.70 & 34.95 & 27.77 \\
\hline 701 & 5.06 & 35.01 & 27.68 & 1781 & 3.70 & 34.95 & 27.78 \\
\hline
\end{tabular}




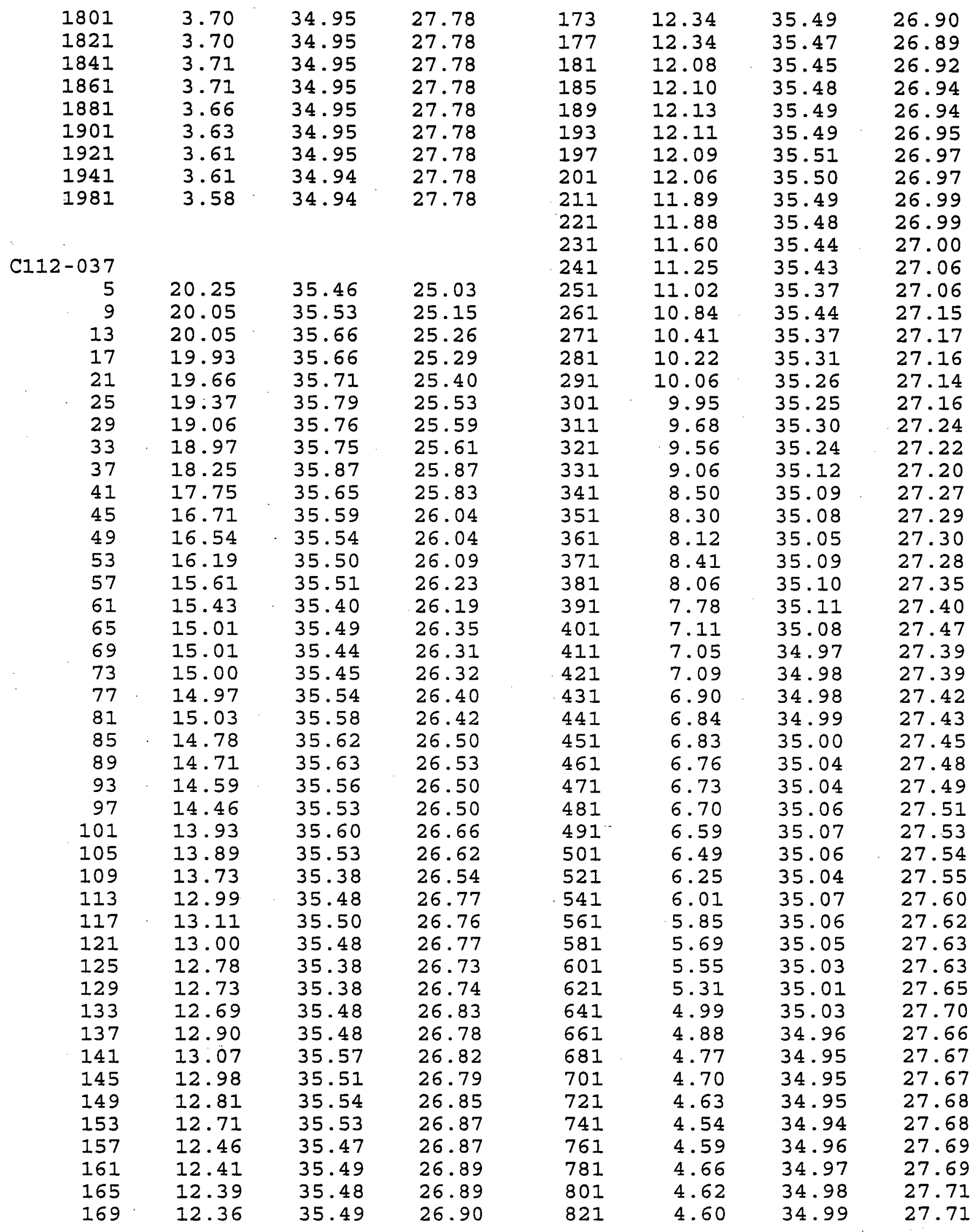




\begin{tabular}{|c|c|c|c|c|c|c|c|}
\hline $\begin{array}{l}841 \\
861 \\
881 \\
901 \\
921 \\
941\end{array}$ & $\begin{array}{l}4.54 \\
4.50 \\
4.48 \\
4.45 \\
4.44 \\
4.42\end{array}$ & $\begin{array}{l}34.98 \\
34.98 \\
34.97 \\
34.98 \\
34.98 \\
34.97\end{array}$ & $\begin{array}{l}27.71 \\
27.71 \\
27.71 \\
27.72 \\
27.72 \\
27.72\end{array}$ & $\begin{array}{l}1921 \\
1941 \\
1981\end{array}$ & $\begin{array}{l}3.63 \\
3.62 \\
3.60\end{array}$ & $\begin{array}{l}34.96 \\
34.96 \\
34.96\end{array}$ & $\begin{array}{l}27.79 \\
27.79 \\
27.79\end{array}$ \\
\hline $\begin{array}{l}961 \\
981\end{array}$ & $\begin{array}{l}4.39 \\
4.34\end{array}$ & $\begin{array}{l}34.98 \\
34.97\end{array}$ & $\begin{array}{l}27.73 \\
27.73\end{array}$ & $\begin{array}{r}9 \\
13\end{array}$ & $\begin{array}{l}21.36 \\
21.35\end{array}$ & $\begin{array}{l}36.27 \\
36.27\end{array}$ & $\begin{array}{l}25.36 \\
25.37\end{array}$ \\
\hline $\begin{array}{l}1001 \\
1021\end{array}$ & $\begin{array}{l}4.31 \\
4.29\end{array}$ & $\begin{array}{l}34.97 \\
34.97\end{array}$ & $\begin{array}{l}27.73 \\
27.73\end{array}$ & $\begin{array}{l}17 \\
21\end{array}$ & 21.36 & 36.28 & 25.37 \\
\hline 1041 & 4.28 & 34.97 & 27.73 & $\begin{array}{l}21 \\
25\end{array}$ & $\begin{array}{l}21.38 \\
21.40\end{array}$ & $\begin{array}{l}36.30 \\
36.32\end{array}$ & $\begin{array}{l}25.38 \\
25.39\end{array}$ \\
\hline 1061 & 4.26 & 34.97 & 27.73 & 29 & 21.43 & 36.35 & 25.41 \\
\hline 1081 & 4.24 & 34.97 & 27.73 & 33 & 21.41 & 36.38 & 25.43 \\
\hline 1101 & 4.16 & 34.97 & 27.74 & 37 & 21.34 & 36.39 & 25.46 \\
\hline 1121 & 4.05 & 34.95 & 27.74 & 41 & 21.19 & 36.34 & 25.47 \\
\hline 1141 & 4.02 & 34.94 & 27.74 & 45 & 21.04 & 36.37 & 25.52 \\
\hline 1161 & 3.99 & 34.94 & 27.74 & 49 & 20.86 & 36.31 & 25.53 \\
\hline 1181 & 3.97 & 34.94 & 27.74 & 53 & 20.55 & 36.27 & 25.59 \\
\hline 1201 & 3.95 & 34.94 & 27.74 & 57 & 19.82 & 36.30 & 25.80 \\
\hline 1221 & 3.93 & 34.93 & 27.74 & 61 & 19.75 & 36.16 & 25.71 \\
\hline 1241 & 3.93 & 34.93 & 27.74 & 65 & 18.81 & 36.09 & 25.90 \\
\hline 1261 & 3.92 & 34.93 & 27.74 & 69 & 18.25 & 36.01 & 25.98 \\
\hline 1281 & 3.91 & 34.93 & 27.74 & 73 & 17.67 & 35.71 & 25.90 \\
\hline 1301 & 3.89 & 34.93 & 27.74 & 77 & 17.30 & 35.94 & 26.17 \\
\hline 1321 & 3.85 & 34.93 & 27.74 & 81 & 16.78 & 35.80 & 26.18 \\
\hline 1341 & 3.80 & 34.93 & 27.75 & 85 & 16.41 & 35.84 & 26.30 \\
\hline 1361 & 3.78 & 34.93 & 27.75 & 89 & 16.28 & 35.87 & 26.35 \\
\hline 1381 & 3.77 & 34.92 & 27.75 & 93 & 16.23 & 35.92 & 26.40 \\
\hline 1401 & 3.77 & 34.92 & 27.75 & 97 & 16.36 & 35.96 & 26.40 \\
\hline 1421 & 3.79 & 34.93 & 27.75 & 101 & 16.43 & 35.99 & 26.41 \\
\hline 1441 & 3.81 & 34.94 & 27.75 & 105 & 16.38 & 36.03 & 26.45 \\
\hline 1461 & 3.79 & 34.94 & 27.76 & 109 & 16.42 & 36.07 & 26.47 \\
\hline 1481 & 3.78 & 34.94 & 27.76 & 113 & 16.43 & 36.10 & 26.50 \\
\hline 1501 & 3.69 & 34.92 & 27.76 & 117 & 16.60 & 36.13 & 26.48 \\
\hline 1521 & 3.67 & 34.92 & 27.76 & 121 & 16.50 & 36.15 & 26.52 \\
\hline 1541 & 3.67 & 34.92 & 27.76 & 125 & 16.41 & 36.14 & 26.53 \\
\hline 1561 & 3.67 & 34.93 & 27.76 & 129 & 16.26 & 36.13 & 26.56 \\
\hline 1581 & 3.67 & 34.93 & 27.76 & 133 & 16.16 & 36.06 & 26.52 \\
\hline 1601 & 3.67 & 34.93 & 27.76 & 137 & 15.81 & 36.06 & 26.61 \\
\hline 1621 & 3.67 & 34.93 & 27.76 & 141 & 15.96 & 36.10 & 26.61 \\
\hline 1641 & 3.67 & 34.93 & 27.77 & 145 & 15.94 & 36.11 & 26.62 \\
\hline 1661 & 3.68 & 34.94 & 27.77 & 149 & 15.89 & 36.09 & 26.61 \\
\hline 1681 & 3.70 & 34.94 & 27.77 & 153 & 15.80 & 36.11 & 26.65 \\
\hline 1701 & 3.70 & 34.95 & 27.78 & 157 & 15.72 & 36.07 & 26.64 \\
\hline 1721 & 3.70 & 34.95 & 27.78 & 161 & 15.61 & 36.06 & 26.6 \\
\hline 1741 & 3.64 & 34.94 & 27.78 & 165 & 15.53 & 36.07 & 26.6 \\
\hline 1761 & 3.65 & 34.94 & 27.78 & 169 & 15.32 & 36.03 & 26.70 \\
\hline 1781 & 3.66 & 34.95 & 27.78 & 173 & 15.28 & 36.05 & 26.7 \\
\hline 1801 & 3.65 & 34.95 & 27.78 & 177 & 15.21 & 36.00 & 26.6 \\
\hline 1821 & 3.66 & 34.95 & 27.78 & 181 & 15.03 & 35.97 & 26.7 \\
\hline 1841 & 3.66 & 34.95 & 27.78 & 185 & 14.78 & 35.90 & 26.7 \\
\hline 1861 & 3.65 & 34.95 & 27.79 & 189 & 14.14 & 35.77 & 26.7 \\
\hline 1881 & 3.64 & 34.95 & 27.79 & 193 & 13.69 & 35.76 & 26.84 \\
\hline 1901 & 3.64 & 34.95 & 27.79 & 197 & 12.94 & 35.65 & 26.91 \\
\hline
\end{tabular}




\begin{tabular}{|c|c|c|c|c|c|c|c|}
\hline 201 & 12.85 & 35.52 & 26.83 & 981 & 4.42 & 34.98 & 27.72 \\
\hline 211 & 12.93 & 35.59 & 26.87 & 1001 & 4.39 & 34.98 & 27.73 \\
\hline 221 & 12.60 & 35.55 & 26.90 & 1021 & 4.36 & 34.97 & 27.73 \\
\hline 231 & 12.35 & 35.50 & 26.91 & 1041 & 4.33 & 34.97 & 27.73 \\
\hline 241 & 12.03 & 35.49 & 26.97 & 1061 & 4.28 & 34.97 & 27.73 \\
\hline 251 & 11.88 & 35.48 & 26.98 & 1081 & 4.25 & 34.97 & 27.74 \\
\hline 261 & 11.72 & 35.46 & 27.00 & 1101 & 4.23 & 34.96 & 27.73 \\
\hline 271 & 11.16 & 35.45 & 27.10 & 1121 & 4.21 & 34.96 & 27.74 \\
\hline 281 & 11.04 & 35.32 & 27.02 & 1141 & 4.19 & 34.96 & 27.74 \\
\hline 291 & 10.85 & 35.37 & 27.09 & 1161 & 4.18 & 34.96 & 27.74 \\
\hline 301 & 10.48 & 35.34 & 27.14 & 1181 & 4.17 & 34.96 & 27.74 \\
\hline 311 & 10.36 & 35.31 & 27.14 & 1201 & 4.14 & 34.96 & 27.74 \\
\hline 321 & 10.25 & 35.31 & 27.15 & 1221 & 4.11 & 34.96 & 27.74 \\
\hline 331 & 9.95 & 35.26 & 27.17 & 1241 & 4.10 & 34.96 & 27.74 \\
\hline 341 & 9.59 & 35.26 & 27.23 & 1261 & 4.07 & 34.96 & 27.75 \\
\hline 351 & 9.50 & 35.22 & 27.21 & 1281 & 4.06 & 34.96 & 27.75 \\
\hline 361 & 9.31 & 35.20 & 27.23 & 1301 & 4.04 & 34.96 & 27.75 \\
\hline 371 & 9.13 & 35.19 & 27.25 & 1321 & 4.04 & 34.96 & 27.75 \\
\hline 381 & 8.96 & 35.18 & 27.27 & 1341 & 4.02 & 34.96 & 27.75 \\
\hline 391 & 8.79 & 35.17 & 27.28 & 1361 & 4.00 & 34.96 & 27.75 \\
\hline 401 & 8.72 & 35.15 & 27.28 & 1381 & 3.98 & 34.95 & 27.75 \\
\hline 411 & 8.66 & 35.14 & 27.28 & & & & \\
\hline 421 & 8.46 & 35.16 & 27.33 & & & & \\
\hline 431 & 8.18 & 35.11 & 27.33 & C112-039 & & & \\
\hline 441 & 7.93 & 35.11 & 27.37 & 5 & 21.05 & 36.20 & $25: 40$ \\
\hline 451 & 7.88 & 35.09 & 27.36 & 9 & 21.06 & 36.21 & 25.40 \\
\hline 461 & 7.70 & 35.10 & 27.40 & 13 & 21.06 & 36.21 & 25.40 \\
\hline 471 & 7.51 & 35.09 & 27.42 & 17 & 21.06 & 36.21 & 25.40 \\
\hline 481 & 7.40 & 35.10 & 27.44 & 21 & 21.06 & 36.21 & 25.40 \\
\hline 491 & 7.30 & 35.10 & 27.45 & 25 & 21.06 & 36.21 & 25.40 \\
\hline 501 & 7.15 & 35.10 & 27.47 & 29 & 21.06 & 36.21 & 25.40 \\
\hline 521 & 6.84 & 35.10 & 27.52 & 33 & 21.06 & 36.21 & 25.40 \\
\hline 541 & 6.61 & 35.07 & 27.53 & 37 & 21.04 & 36.19 & 25.39 \\
\hline 573 & 6.28 & 35.05 & 27.55 & 41 & 20.55 & 36.17 & 25.51 \\
\hline 593 & 6.04 & 35.05 & 27.59 & 45 & 19.96 & 35.91 & 25.46 \\
\hline 601 & 6.02 & 35.03 & 27.58 & 49 & 18.92 & 36.01 & 25.82 \\
\hline 621 & 5.86 & 35.03 & 27.60 & 53 & 18.39 & 35.50 & 25.56 \\
\hline 641 & 5.65 & 35.03 & 27.62 & 57 & 16.88 & 35.74 & 26.11 \\
\hline 661 & 5.52 & 35.03 & 27.64 & 61 & 16.80 & 36.22 & 26.50 \\
\hline 681 & 5.40 & 35.03 & 27.65 & 65 & 17.67 & 36.07 & 26.17 \\
\hline 701 & 5.28 & 35.03 & 27.66 & 69 & 17.39 & 36.12 & 26.28 \\
\hline 721 & 5.11 & 35.00 & 27.66 & 73 & 16.83 & 36.06 & 26.37 \\
\hline 741 & 4.99 & 35.00 & 27.67 & 77 & 16.65 & 35.99 & 26.36 \\
\hline 761 & 4.90 & 35.00 & 27.68 & 81 & 16.64 & 36.01 & 26.38 \\
\hline 781 & 4.84 & 35.00 & 27.69 & 85 & 16.47 & 36.07 & 26.46 \\
\hline 801 & 4.81 & 35.00 & 27.69 & 89 & 16.49 & 36.08 & 26.47 \\
\hline 821 & 4.73 & 34.99 & 27.70 & 93 & 16.44 & 36.08 & 26.48 \\
\hline 841 & 4.71 & 34.99 & 27.70 & 97 & 16.32 & 36.08 & 26.51 \\
\hline 861 & 4.67 & 34.99 & 27.71 & 101 & 16.33 & 36.13 & 26.54 \\
\hline 881 & 4.62 & 34.99 & 27.71 & 105 & 16.27 & 36.08 & 26.52 \\
\hline 901 & 4.59 & 34.99 & 27.71 & 109 & 15.99 & 36.12 & 26.61 \\
\hline 921 & 4.55 & $34: 99$ & 27.72 & 113 & 15.97 & 36.06 & 26.57 \\
\hline 941 & 4.51 & 34.98 & 27.72 & 117 & 15.92 & 36.04 & 26.57 \\
\hline 961 & 4.47 & 34.98 & 27.72 & 121 & 15.90 & 36.02 & 26.55 \\
\hline
\end{tabular}




\begin{tabular}{|c|c|c|c|c|c|c|c|}
\hline & & & & & & & \\
\hline $\begin{array}{l}125 \\
129\end{array}$ & $\begin{array}{l}15.56 \\
15.49\end{array}$ & $\begin{array}{l}35.98 \\
35.99\end{array}$ & $\begin{array}{l}26.61 \\
26.62\end{array}$ & $\begin{array}{l}601 \\
621\end{array}$ & $\begin{array}{l}5.65 \\
5.56\end{array}$ & $\begin{array}{l}35.02 \\
35.03\end{array}$ & $\begin{array}{l}27.62 \\
27.64\end{array}$ \\
\hline 133 & 15.53 & 35.99 & 26.62 & 641 & 5.41 & 35.01 & $\begin{array}{l}27.64 \\
27.64\end{array}$ \\
\hline 137 & 15.42 & 35.83 & 26.52 & 661 & 5.29 & 35.02 & 27.66 \\
\hline 141 & 15.13 & 35.89 & 26.63 & 681 & 5.23 & 35.01 & 27.66 \\
\hline 145 & 15.04 & 35.93 & 26.68 & 701 & 5.14 & 35.01 & 27.67 \\
\hline 149 & 14.92 & 35.78 & 26.60 & 721 & 4.98 & 35.00 & 27.68 \\
\hline 153 & 14.56 & 35.86 & 26.73 & 741 & 4.90 & 34.99 & 27.68 \\
\hline 157 & 14.52 & 35.82 & 26.71 & 761 & 4.85 & 35.00 & 27.69 \\
\hline 161 & 14.31 & 35.76 & 26.71 & 781 & 4.81 & 34.99 & 27.69 \\
\hline 165 & 14.24 & 35.81 & 26.76 & 801 & 4.77 & 34.99 & 27.69 \\
\hline 169 & 14.38 & 35.86 & 26.77 & 821 & 4.59 & 34.98 & 27.71 \\
\hline 173 & 14.59 & 35.90 & 26.75 & 841 & 4.54 & 34.98 & 27.71 \\
\hline 177 & 14.44 & 35.76 & 26.68 & 861 & 4.47 & 34.98 & 27.72 \\
\hline 181 & 13.99 & 35.75 & 26.77 & 881 & 4.43 & 34.98 & 27.72 \\
\hline 185 & 13.93 & 35.75 & 26.78 & 901 & 4.42 & 34.98 & 27.72 \\
\hline 189 & 13.89 & 35.75 & 26.79 & 921 & 4.41 & 34.97 & 27.72 \\
\hline 193 & 13.81 & 35.70 & 26.77 & 941 & 4.39 & 34.97 & 27.72 \\
\hline 197 & 13.67 & 35.74 & 26.83 & 961 & 4.37 & 34.97 & 27.73 \\
\hline 201 & 13.58 & 35.70 & 26.81 & 981 & 4.34 & 34.97 & 27.73 \\
\hline $21 I$ & 13.41 & 35.71 & 26.86 & 1001 & 4.30 & 34.97 & 27.73 \\
\hline 221 & 13.17 & 35.69 & 26.89 & 1021 & 4.28 & 34.97 & 27.73 \\
\hline 231 & 12.68 & 35.70 & 27.00 & 1041 & 4.22 & 34.97 & 27.74 \\
\hline 241 & 12.54 & 35.61 & 26.95 & 1061 & 4.18 & 34.96 & 27.74 \\
\hline 251 & 12.24 & 35.56 & 26.98 & 1081 & 4.17 & 34.96 & 27.74 \\
\hline 261 & 11.77 & 35.50 & 27.02 & 1101 & 4.15 & 34.96 & 27.74 \\
\hline 271 & 11.61 & 35.48 & 27.03 & 1121 & 4.13 & 34.96 & 27.74 \\
\hline 281 & 11.43 & 35.43 & 27.03 & 1141 & 4.12 & 34.96 & 27.74 \\
\hline 291 & 11.25 & 35.41 & 27.05 & 1161 & 4.12 & 34.96 & 27.74 \\
\hline 301 & 10.80 & 35.36 & 27.09 & 1181 & 4.12 & 34.95 & 27.74 \\
\hline 311 & 10.68 & 35.33 & 27.09 & 1201 & 4.09 & 34.96 & 27.75 \\
\hline 321 & 10.36 & 35.26 & 27.10 & 1221 & 4.05 & 34.95 & 27.74 \\
\hline 331 & 10.17 & 35.30 & 27.16 & 1241 & 4.02 & 34.96 & 27.75 \\
\hline 341 & 9.81 & 35.27 & 27.20 & 1261 & 4.01 & 34.95 & 27.75 \\
\hline 351 & 9.47 & 35.22 & 27.21 & 1281 & 4.01 & 34.95 & 27.75 \\
\hline 361 & 9.40 & 35.22 & 27.23 & 1301 & 4.01 & 34.96 & 27.75 \\
\hline 371 & 9.02 & 35.17 & 27.25 & 1321 & 3.98 & 34.95 & 27.75 \\
\hline 381 & 8.78 & 35.16 & 27.28 & 1341 & 3.95 & 34.95 & 27.76 \\
\hline 391 & 8.55 & 35.11 & 27.28 & 1361 & 3.93 & 34.95 & 27.76 \\
\hline 401 & 8.53 & 35.11 & 27.28 & 1381 & 3.91 & 34.96 & 27.76 \\
\hline 411 & 8.43 & 35.13 & 27.31 & 1401 & 3.89 & 34.96 & 27.76 \\
\hline 421 & 8.32 & 35.10 & 27.30 & 1421 & 3.88 & 34.96 & 27.76 \\
\hline 431 & 8.25 & 35.08 & 27.30 & 1441 & 3.87 & 34.96 & 27.76 \\
\hline 441 & 7.80 & 35.09 & 27.37 & 1461 & 3.85 & 34.96 & 27.77 \\
\hline 451 & 7.75 & 35.10 & 27.39 & 1481 & 3.84 & 34.96 & 27.77 \\
\hline 461 & 7.56 & 35.06 & 27.39 & 1501 & 3.83 & 34.96 & 27.77 \\
\hline 471 & 7.42 & 35.07 & 27.42 & 1521 & 3.82 & 34.96 & 27.77 \\
\hline 481 & 7.28 & 35.08 & 27.44 & 1541 & 3.82 & 34.96 & 27.77 \\
\hline 491 & 7.04 & 35.03 & 27.44 & 1561 & 3.81 & 34.96 & 27.77 \\
\hline 501 & 6.85 & 35.06 & 27.49 & 1581 & 3.80 & 34.96 & 27.77 \\
\hline 521 & 6.57 & 35.04 & 27.51 & 1601 & 3.79 & 34.96 & 27.78 \\
\hline 541 & 6.22 & 35.04 & 27.56 & 1621 & 3.78 & 34.96 & 27.78 \\
\hline 561 & 6.08 & 35.03 & 27.57 & 1641 & 3.75 & 34.96 & 27.78 \\
\hline 581 & 5.86 & 35.04 & 27.60 & 1661 & 3.75 & 34.96 & 27.78 \\
\hline
\end{tabular}




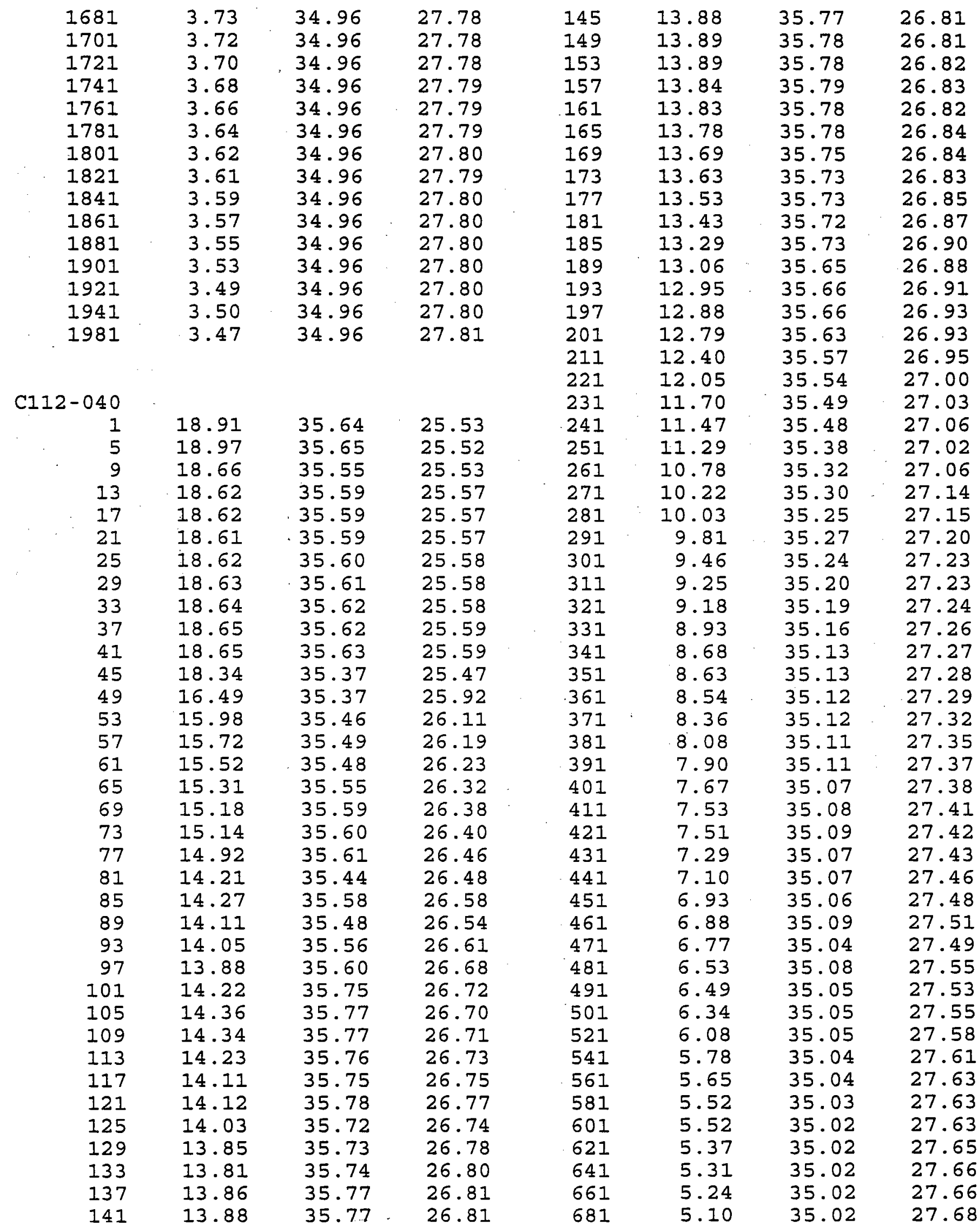




\begin{tabular}{|c|c|c|c|c|c|c|c|}
\hline $\begin{array}{l}701 \\
721 \\
741 \\
761 \\
781\end{array}$ & $\begin{array}{l}5.00 \\
4.87 \\
4.82 \\
4.69 \\
4.62\end{array}$ & $\begin{array}{l}35.01 \\
35.00 \\
35.00 \\
34.98 \\
34.97\end{array}$ & $\begin{array}{l}27.68 \\
27.69 \\
27.70 \\
27.70 \\
27.70\end{array}$ & $\begin{array}{l}1781 \\
1801\end{array}$ & $\begin{array}{l}3.66 \\
3.64\end{array}$ & $\begin{array}{l}34.96 \\
34.96\end{array}$ & $\begin{array}{l}27.79 \\
27.79\end{array}$ \\
\hline 801 & 4.55 & 34.98 & 27.71 & 1 & 9.34 & 31.32 & 24.19 \\
\hline $\begin{array}{l}821 \\
841\end{array}$ & $\begin{array}{l}4.57 \\
4.61\end{array}$ & $\begin{array}{l}34.98 \\
34.99\end{array}$ & $\begin{array}{l}27.71 \\
27.72\end{array}$ & $\begin{array}{l}5 \\
9\end{array}$ & $\begin{array}{l}9.34 \\
8.89\end{array}$ & $\begin{array}{l}31.32 \\
31.30\end{array}$ & $\begin{array}{l}24.19 \\
24.24\end{array}$ \\
\hline 861 & 4.53 & 34.99 & 27.72 & 13 & $\begin{array}{l}8.89 \\
7.29\end{array}$ & $\begin{array}{l}31.30 \\
31.47\end{array}$ & $\begin{array}{l}24.24 \\
24.60\end{array}$ \\
\hline 881 & 4.49 & 34.98 & 27.72 & 17 & 6.95 & 31.30 & 24.51 \\
\hline 901 & 4.46 & 34.98 & 27.72 & 21 & 6.52 & 31.43 & 24.67 \\
\hline 921 & 4.46 & 34.98 & 27.72 & 25 & 6.25 & 31.46 & 24.73 \\
\hline 941 & 4.42 & 34.99 & 27.73 & 29 & 6.42 & 31.60 & 24.81 \\
\hline 961 & 4.39 & 34.98 & 27.73 & 33 & 5.90 & 31.60 & 24.88 \\
\hline 981 & 4.35 & 34.98 & 27.73 & 37 & 5.46 & 31.87 & 25.14 \\
\hline 1001 & 4.33 & 34.98 & 27.73 & 41 & 5.20 & 32.07 & 25.33 \\
\hline 1021 & 4.28 & 34.98 & 27.74 & 45 & 4.80 & 32.18 & 25.46 \\
\hline 1041 & 4.24 & 34.98 & 27.74 & 49 & 4.39 & 32.33 & 25.63 \\
\hline 1061 & 4.21 & 34.97 & 27.74 & 53 & 3.29 & 32.39 & 25.77 \\
\hline 1081 & 4.15 & 34.96 & 27.74 & 57 & 2.92 & 32.26 & 25.71 \\
\hline 1101 & 4.15 & 34.97 & 27.74 & 61 & 2.26 & 32.32 & 25.81 \\
\hline 1121 & 4.10 & 34.96 & 27.74 & 65 & 2.01 & 32.25 & 25.77 \\
\hline 1141 & 4.07 & 34.96 & 27.75 & 69 & 2.92 & 32.60 & 25.97 \\
\hline 1161 & 4.05 & 34.96 & 27.75 & 73 & 3.87 & 32.85 & 26.09 \\
\hline 1181 & 4.04 & 34.96 & 27.75 & 77 & 4.30 & 33.17 & 26.30 \\
\hline 1201 & 4.02 & 34.96 & 27.75 & 81 & 4.37 & 33.22 & 26.33 \\
\hline 1221 & 4.01 & 34.96 & 27.75 & & & & \\
\hline 1241 & 3.98 & 34.96 & 27.76 & & . & & \\
\hline 1261 & 3.95 & 34.96 & 27.76 & & & & \\
\hline 1281 & 3.94 & 34.96 & 27.76 & & & & \\
\hline 1301 & 3.93 & 34.96 & 27.76 & & & & \\
\hline 1321 & 3.92 & $34 \cdot 9.6$ & 27.76 & & & & \\
\hline 1341 & 3.91 & 34.96 & 27.76 & & & & \\
\hline 1361 & 3.89 & 34.96 & 27.76 & & & & \\
\hline 1381 & 3.89 & 34.96 & 27.76 & & & & \\
\hline 1401 & 3.89 & 34.96 & 27.76 & & & & \\
\hline 1421 & 3.88 & 34.96 & 27.77 & & & & \\
\hline 1485 & 3.84 & 34.96 & 27.77 & & & & \\
\hline 1505 & 3.82 & 34.96 & 27.77 & & : & & \\
\hline 1481 & 3.84 & 34.96 & 27.77 & & & & \\
\hline 1501 & 3.82 & 34.96 & 27.77 & & & & \\
\hline 1521 & 3.81 & 34.96 & 27.77 & & & & \\
\hline 1541 & 3.80 & 34.96 & 27.78 & & & & \\
\hline 1561 & 3.78 & 34.96 & 27.78 & & & & \\
\hline 1581 & 3.77 & 34.96 & 27.78 & & & & \\
\hline 1601 & 3.76 & 34.96 & 27.78 & & & & \\
\hline 1621 & 3.75 & 34.96 & 27.78 & & & & \\
\hline 1641 & 3.74 & 34.96 & 27.78 & & & & \\
\hline 1661 & 3.73 & 34.96 & 27.78 & & & & \\
\hline 1681 & 3.72 & 34.96 & 27.78 & & & & \\
\hline 1701 & 3.71 & 34.96 & 27.79 & & & & \\
\hline 1721 & 3.69 & 34.96 & 27.79 & & & & \\
\hline 1741 & 3.68 & 34.96 & 27.79 & & & & \\
\hline 1761 & 3.67 & 34.96 & 27.79 & & & & \\
\hline
\end{tabular}


Table VIII. Chemical data from all hydrocast stations taken during SSV Corwith Cramer Cruise C-II2. AOU= apparent oxygen utilization

STATION
DEPTH

C112-001

0
5
10
15
20
30

C112-002

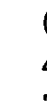

0
4
7

10

16

25

35

50

75

C112-003

$\begin{array}{rr}0 & 0.33 \\ 10 & 0.24 \\ 20 & 0.39 \\ 30 & 0.74 \\ 50 & 2.12 \\ 70 & 1.09\end{array}$

C112-004

$$
\begin{array}{r}
0 \\
10 \\
20 \\
30 \\
40 \\
50 \\
75 \\
100
\end{array}
$$

C112-005

0
9
18
27
36
45
68
90

C112-006

0
10
20
30
45

0.48

0.45

0.66

0.72

0.82

1.03

0.95

1. 21

0.38

0.75

0.66

1.09

0.83

0.94

1. 34

0.71

0.61

0.68

1.15

0.55

0.57

0.62

10
15

$$
\text { C112-007 }
$$

$\begin{array}{rr}0 & 0.57 \\ 10 & 0.62 \\ 15 & 0.85\end{array}$

$\begin{array}{lc}\text { CHL } & \text { SILICATE } \\ \mathrm{ug} / \mathrm{L} & \mathrm{uM} / \mathrm{L}\end{array}$

0.27

0.11

0.12

0.26

0.32

0.27

0.65

0.09

0.43

0.42

0.92

0.40

0.14

0.26

1.00

1. 37

0.42

0.57

0.33

0.07

$\begin{array}{lr} & 1.94 \\ 0.16 & 0.84 \\ 0.82 & 5.36 \\ 0.53 & 3.76 \\ 0.11 & 9.35 \\ 0.09 & 6.22 \\ 0.05 & 10.10 \\ 0.03 & 13.65\end{array}$

0.07

1.08

0.75

1.02

0.48

0.77

0.66

0.42

0.71

0.68

0.39

0.45

0.84

1.08
0.89
0.69

2.89

6.28

4.61

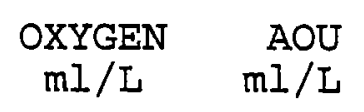

6.67

6.87

6.51

6.97

6.79

7.52

7.41

7.84

7.34

7.66

7.43

7.22

7.20

6.82

7.39

7.49

6.74

6.64

6.21

6.33 


\begin{tabular}{|c|c|c|c|c|c|c|}
\hline STATION & $\begin{array}{r}\text { DEPTH } \\
20 \\
25 \\
35\end{array}$ & $\begin{array}{l}P 04 \\
0.77 \\
0.70 \\
0.44\end{array}$ & $\begin{array}{l}\text { CHL } \\
1.07 \\
1.25\end{array}$ & $\begin{array}{r}\text { SILICATE } \\
4.09 \\
6.38 \\
20.70\end{array}$ & OXYGEN & AOU \\
\hline C112-008 & $\begin{array}{r}0 \\
5 \\
10 \\
17 \\
25 \\
35\end{array}$ & $\begin{array}{l}0.62 \\
0.62 \\
0.54 \\
0.63 \\
0.58 \\
0.60\end{array}$ & $\begin{array}{l}0.70 \\
0.80 \\
0.72 \\
0.89 \\
0.92 \\
0.83\end{array}$ & & & \\
\hline C112-009 & $\begin{array}{r}0 \\
5 \\
10 \\
20 \\
30 \\
40\end{array}$ & $\begin{array}{l}0.55 \\
0.48 \\
0.43 \\
0.60 \\
0.69 \\
0.91\end{array}$ & $\begin{array}{l}0.45 \\
0.69 \\
0.36 \\
0.57 \\
0.81\end{array}$ & & & \\
\hline C112-010 & $\begin{array}{r}0 \\
3 \\
5 \\
8 \\
12 \\
18 \\
30 \\
40\end{array}$ & $\begin{array}{l}0.41 \\
0.41 \\
0.37 \\
0.32 \\
0.34 \\
0.51 \\
0.40 \\
0.45\end{array}$ & $\begin{array}{l}0.76 \\
0.67 \\
1.18 \\
1.83 \\
0.52 \\
1.07 \\
0.56 \\
1.27\end{array}$ & & & \\
\hline C112-023 & $\begin{array}{r}0 \\
50 \\
100 \\
150 \\
300 \\
500 \\
750 \\
1000 \\
1500 \\
2000 \\
2500 \\
3000\end{array}$ & $\begin{array}{l}0.05 \\
0.09 \\
0.08 \\
0.15 \\
0.16 \\
0.45 \\
0.23 \\
0.65 \\
1.29 \\
1.16 \\
1.20 \\
1.21\end{array}$ & $\begin{array}{l}0.02 \\
0.05 \\
0.22 \\
0.19 \\
0.00\end{array}$ & & $\begin{array}{l}5.22 \\
5.27 \\
5.09 \\
5.07 \\
5.27 \\
5.08 \\
5.31 \\
4.88 \\
5.19 \\
6.08 \\
6.21 \\
6.29\end{array}$ & $\begin{array}{r}-0.12 \\
-0.17 \\
0.11 \\
0.18 \\
0.08 \\
0.42 \\
0.69 \\
1.92 \\
2.06 \\
1.22 \\
1.24 \\
1.21\end{array}$ \\
\hline
\end{tabular}

$\begin{array}{rrrr}\text { C112-034 } & 0 & 0.08 & 0.10 \\ & 46 & 0.13 & 0.08 \\ & 92 & 0.18 & 0.10 \\ 231 & 0.10 & 0.04 \\ 458 & 0.21 & \\ & 724 & 1.03 & \\ & 1253 & 1.23 & \\ & 1770 & 0.96 & \\ \text { C112-035 } & 0 & 0.00 & 0.17 \\ & 22 & & 0.21 \\ & 45 & 0.04 & 0.20 \\ & 110 & 0.35 & 0.14\end{array}$




\begin{tabular}{|c|c|c|c|c|c|}
\hline STATION & $\begin{array}{r}\text { DEPTH } \\
200 \\
320 \\
545 \\
775\end{array}$ & $\begin{array}{l}\text { PO4 } \\
0.76 \\
1.45 \\
1.64 \\
1.06\end{array}$ & CHI & SILICATE & OXYGEN \\
\hline C112-036 & $\begin{array}{r}0 \\
15 \\
30 \\
60 \\
120 \\
250\end{array}$ & $\begin{array}{r}-0.02 \\
0.12 \\
0.17 \\
0.44 \\
0.71 \\
1.09\end{array}$ & $\begin{array}{l}0.17 \\
0.22 \\
0.19 \\
0.09 \\
0.01 \\
0.00\end{array}$ & & \\
\hline C112-037 & $\begin{array}{r}0 \\
15 \\
30 \\
60 \\
120 \\
250\end{array}$ & $\begin{array}{l}0.13 \\
0.11 \\
0.23 \\
0.75 \\
1.31\end{array}$ & $\begin{array}{l}0.12 \\
0.13 \\
0.24 \\
0.08 \\
0.00\end{array}$ & & \\
\hline C112-038 & $\begin{array}{r}0 \\
14 \\
27 \\
54 \\
108 \\
225\end{array}$ & $\begin{array}{r}0.21 \\
0.01 \\
-0.01 \\
0.26 \\
0.42 \\
1.16\end{array}$ & $\begin{array}{l}0.16 \\
0.15 \\
0.17 \\
0.31 \\
0.26 \\
0.01\end{array}$ & & \\
\hline C112-039 & $\begin{array}{r}0 \\
15 \\
30 \\
60 \\
120 \\
250\end{array}$ & $\begin{array}{l}0.08 \\
0.08 \\
0.12 \\
0.38 \\
0.72 \\
1.11\end{array}$ & $\begin{array}{l}0.23 \\
0.27 \\
0.23 \\
0.20 \\
0.02 \\
0.00\end{array}$ & & \\
\hline C112-040 & $\begin{array}{r}0 \\
13 \\
26 \\
52 \\
104 \\
216 \\
432 \\
888 \\
1330 \\
1790\end{array}$ & $\begin{array}{l}0.16 \\
0.05 \\
0.01 \\
0.44 \\
0.82 \\
1.23 \\
1.37 \\
1.28 \\
1.14 \\
1.25\end{array}$ & $\begin{array}{l}0.17 \\
0.27 \\
0.30 \\
0.19\end{array}$ & & $\begin{array}{l}5.87 \\
5.64 \\
5.46 \\
5.14 \\
4.30 \\
3.80 \\
4.51 \\
5.91 \\
5.93 \\
6.24\end{array}$ \\
\hline C112-046 & $\begin{array}{r}0 \\
10 \\
25 \\
50 \\
75 \\
110\end{array}$ & $\begin{array}{l}0.36 \\
0.41 \\
0.35 \\
1.29 \\
0.93 \\
1.28\end{array}$ & $\begin{array}{l}0.16 \\
0.15 \\
0.23 \\
0.05 \\
0.01 \\
0.01\end{array}$ & & \\
\hline C112-048 & $\begin{array}{l}0 \\
6\end{array}$ & $\begin{array}{l}0.45 \\
0.38\end{array}$ & $\begin{array}{l}0.08 \\
0.09\end{array}$ & & $\begin{array}{l}5.48 \\
7.17\end{array}$ \\
\hline
\end{tabular}




\begin{tabular}{|c|c|c|c|c|c|}
\hline STATION & $\begin{array}{r}\text { DEPTH } \\
11 \\
17 \\
27 \\
41 \\
60 \\
80\end{array}$ & $\begin{array}{l}\text { PO4 } \\
0.36 \\
0.49 \\
1.00 \\
0.45 \\
0.55 \\
1.19\end{array}$ & $\begin{array}{l}\text { CHL } \\
0.19 \\
0.10 \\
0.15 \\
0.09 \\
0.12 \\
0.01\end{array}$ & SIIICATE & $\begin{array}{r}\text { OXYGEN } \\
7.26 \\
7.33 \\
7.66 \\
7.39 \\
7.46 \\
7.14\end{array}$ \\
\hline C112-062 & $\begin{array}{r}0 \\
7 \\
12 \\
20 \\
35 \\
60\end{array}$ & $\begin{array}{l}0.16 \\
0.38 \\
0.38 \\
0.48 \\
0.78 \\
1.22\end{array}$ & $\begin{array}{l}0.82 \\
0.89 \\
0.57 \\
1.55 \\
0.21 \\
0.07\end{array}$ & $\therefore$ & $\begin{array}{l}3.92 \\
3.73 \\
4.40 \\
4.32 \\
4.25 \\
5.06\end{array}$ \\
\hline CI12-064 & $\begin{array}{r}0 \\
10 \\
20 \\
30 \\
50 \\
80 \\
130\end{array}$ & $\begin{array}{l}0.34 \\
0.78 \\
0.17 \\
1.64 \\
1.75 \\
2.88\end{array}$ & $\begin{array}{l}0.81 \\
0.78 \\
1.14 \\
0.48 \\
0.12 \\
0.09 \\
0.61\end{array}$ & 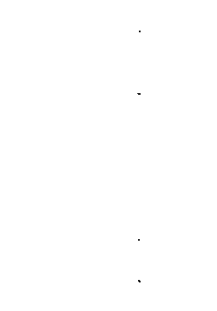 & $\begin{array}{l}7.01 \\
7.82 \\
7.00 \\
6.73 \\
5.51 \\
6.50 \\
6.31\end{array}$ \\
\hline$C 112-066$ & $\begin{array}{r}0 \\
10 \\
20 \\
30 \\
50 \\
80 \\
150 \\
240\end{array}$ & $\begin{array}{l}0.63 \\
0.14 \\
0.09 \\
0.85 \\
0.96 \\
0.93 \\
1.19 \\
1.23\end{array}$ & $\begin{array}{l}0.29 \\
0.31 \\
\\
1.18 \\
0.68 \\
0.10 \\
0.01 \\
0.01\end{array}$ & & $\begin{array}{l}6.73 \\
6.47 \\
6.78 \\
7.23 \\
6.86 \\
6.70 \\
6.86 \\
5.39\end{array}$ \\
\hline
\end{tabular}


Table IX. Meter Net Data

$\begin{array}{crr}\text { Station } & \text { Depth }(\mathrm{m}) & \text { Biomass }\left(\mathrm{ml} / 1000 \mathrm{~m}^{3}\right) \\ 1 & & \\ 2 & 27 & 940 \\ 3 & 100 & 1140 \\ 4 & 70 & 801 \\ 5 & 175 & 1265 \\ 7 & 152 & 203 \\ 9 & 27 & 100 \\ 10 & 30 & 170 \\ 11 & 20 & 249 \\ 13 & 35 & 1660 \\ 16 & 25 & 415 \\ 17 & 50 & 219 \\ 21 & 200 & 6 \\ 22 & 200 & 89 \\ 26 & 200 & 102 \\ 29 & 200 & 33 \\ 39 & 200 & 79 \\ 46 & 200 & 1810 \\ 48 & 100 & 1460 \\ 49 & 40 & 553 \\ 62 & 40 & 511 \\ 64 & 60 & 600 \\ 66 & 100 & 281 \\ 68 & 200 & 273 \\ 69 & 60 & 1110 \\ & 35 & 150\end{array}$


Table X. Neuston Net Data

\begin{tabular}{|c|c|c|c|c|}
\hline Station & Biomass $\left(\mathrm{ml} / 1000 \mathrm{~m}^{3}\right)$ & $\operatorname{Tar}\left(\mathrm{mg} / 1000 \mathrm{~m}^{2}\right)$ & $\begin{array}{l}\text { Plastic } \\
\text { Pieces }\end{array}$ & $\begin{array}{l}\text { Plastic } \\
\text { Pellets }\end{array}$ \\
\hline 1 & 216 & 0 & 0 & 0 \\
\hline 2 & 22 & 0 & 0 & \\
\hline 3 & 11 & 0 & 0 & 0 \\
\hline 4 & 16 & 0 & 0 & 0 \\
\hline 8 & 8 & 0 & 1 & 0 \\
\hline 9 & 11 & 0 & 0 & 0 \\
\hline 15 & 1 & 0.04 & 1 & 1 \\
\hline 17 & 22 & 0.36 & 2 & 0 \\
\hline 19 & 13 & 0.16 & 1 & 0 \\
\hline 21 (tow lost) & - & - & - & - \\
\hline 22 & 3 & 0.60 & 1 & 3 \\
\hline 24 & 18 & 0.80 & 7 & 17 \\
\hline 25 & 16 & 5.92 & 7 & 24 \\
\hline 26 & 14 & 0.16 & 0 & 14 \\
\hline 28 & 3 & 2.26 & 6 & 1 \\
\hline 29 & 22 & 0.64 & 3 & 5 \\
\hline 30 (tow lost) & - & - & - & - \\
\hline 31 & 16 & 0.60 & 1 & 3 \\
\hline 33 & 5 & 0 & 0 & 0 \\
\hline 36 & 26 & 0 & 0 & 0 \\
\hline 39 & 32 & 0 & 0 & 0 \\
\hline 41 & 51 & 0 & 1 & 0 \\
\hline 42 & 2 & 0.26 & 1 & 1 \\
\hline 43 & 254 & 0 & 0 & 0 \\
\hline 44 & 16 & 0 & 0 & 0 \\
\hline 46 & 1 & 0 & 1 & 1 \\
\hline 47 & 562 & 0 & 2 & 0 \\
\hline 49 & 15 & 0 & 0 & 0 \\
\hline 52 & 49 & 0 & \multicolumn{2}{|c|}{ not measured } \\
\hline 53 & - & 0 & \multicolumn{2}{|c|}{ not measured } \\
\hline 54 & 19 & 0 & \multicolumn{2}{|c|}{ not measured } \\
\hline 55 & 1 & 0 & \multicolumn{2}{|c|}{ not measured } \\
\hline 56 & 17 & 0 & \multicolumn{2}{|c|}{ not measured } \\
\hline 57 & 80 & 0 & \multicolumn{2}{|c|}{ not measured } \\
\hline 58 & 65 & 0 & \multicolumn{2}{|c|}{ not measured } \\
\hline 61 & 184 & 0 & & sured \\
\hline
\end{tabular}




\section{Horizontal Distributions from Surface Samples}

The different oceanographic regimes encountered during C-112 are clearly shown by the horizontal distribution of temperature, salinity, dissolved inorganic phosphate, and chlorophyll at the surface (Figures 4-6, temperature not shown). Surface samples were routinely collected throughout the cruise at regular intervals with the exception of a rather stormy passage across the Gulf Stream heading to Bermuda. The results presented here are from the surface station data and the surface samples from hydro stations HC-4, 9, 10, 32, 37, 38, 39, 40, 48, 62, 64, and 66. The salinity distribution (Figure 4) shows most clearly the separate regions of Nantucket Shoals-Georges Bank, Slope Water, Sargasso Sea, and Scotian Shelf. The Gulf of Maine is separated into a central region of moderate salinity $(\sim 32.7 \%)$ and a western section which has lower salinity $(<32 \%)$ and is warmer. This area is influenced by the river runoff to the north and is often an area affected by toxic algal blooms in the summer. In addition two stations just south of Georges Bank showed the presence of a warm-core eddy. The average values for the various properties are given in Table XI.

In general phosphate and chlorophyll are correlated as both decline from the shelf regions to the Slope Water and to the Sargasso Sea. However, there are some interesting exceptions. The phosphate levels on the Scotian Shelf are similar to those on Georges Bank but the chlorophyll values are much lower suggesting that something else must be limiting phytoplankton biomass. The warm core eddy also has very high phosphate while chlorophyll remains low. The differences between Georges Bank and the Gulf of Maine will be discussed further in the next section in a comparison of their plankton dynamics and the relative importance of nutrient recycling and "new" nutrients. 


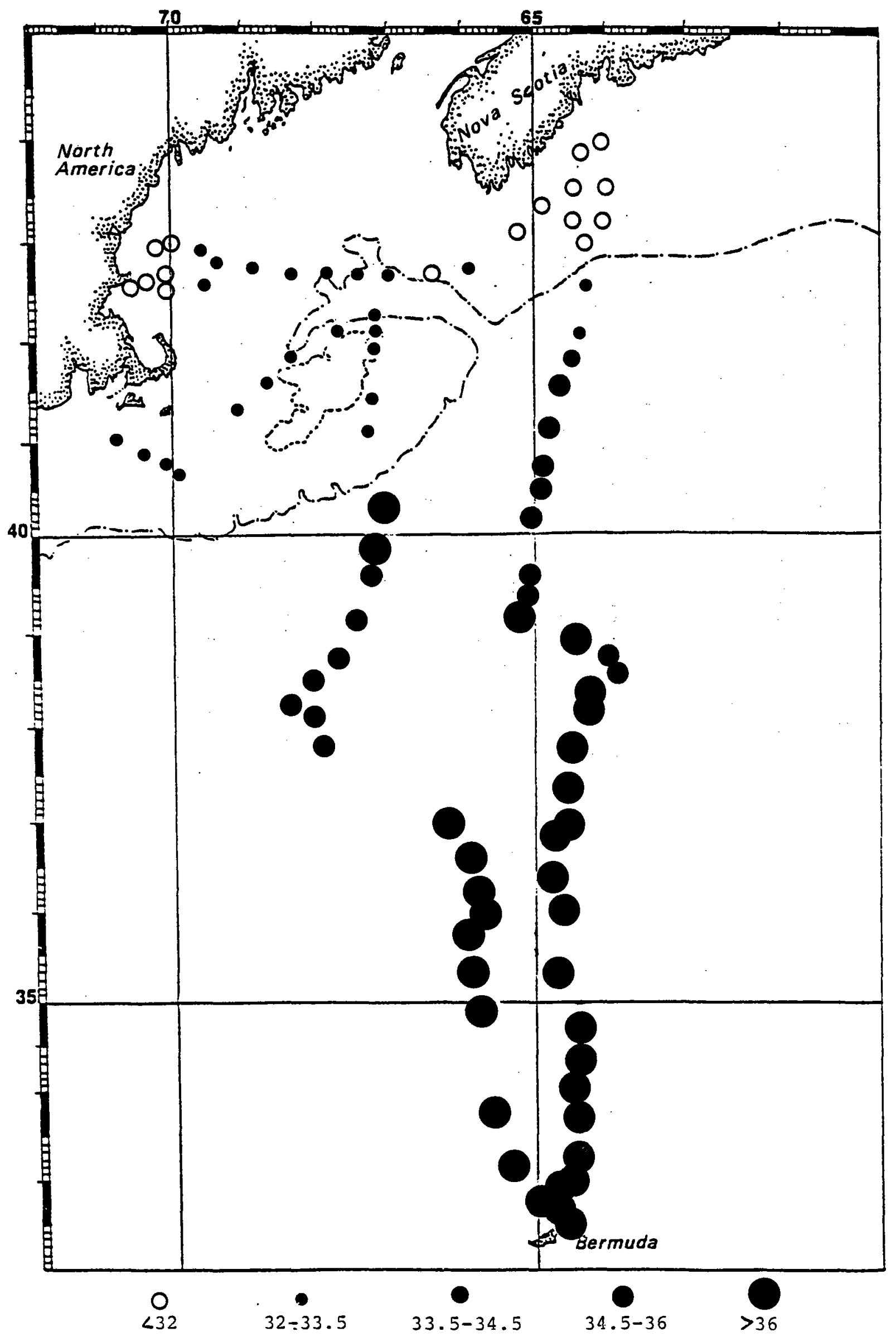

Figure 4 Horizontal distribution of surface salinity for SsV Corwith Cramer Cruise C-112 (0/oo). 


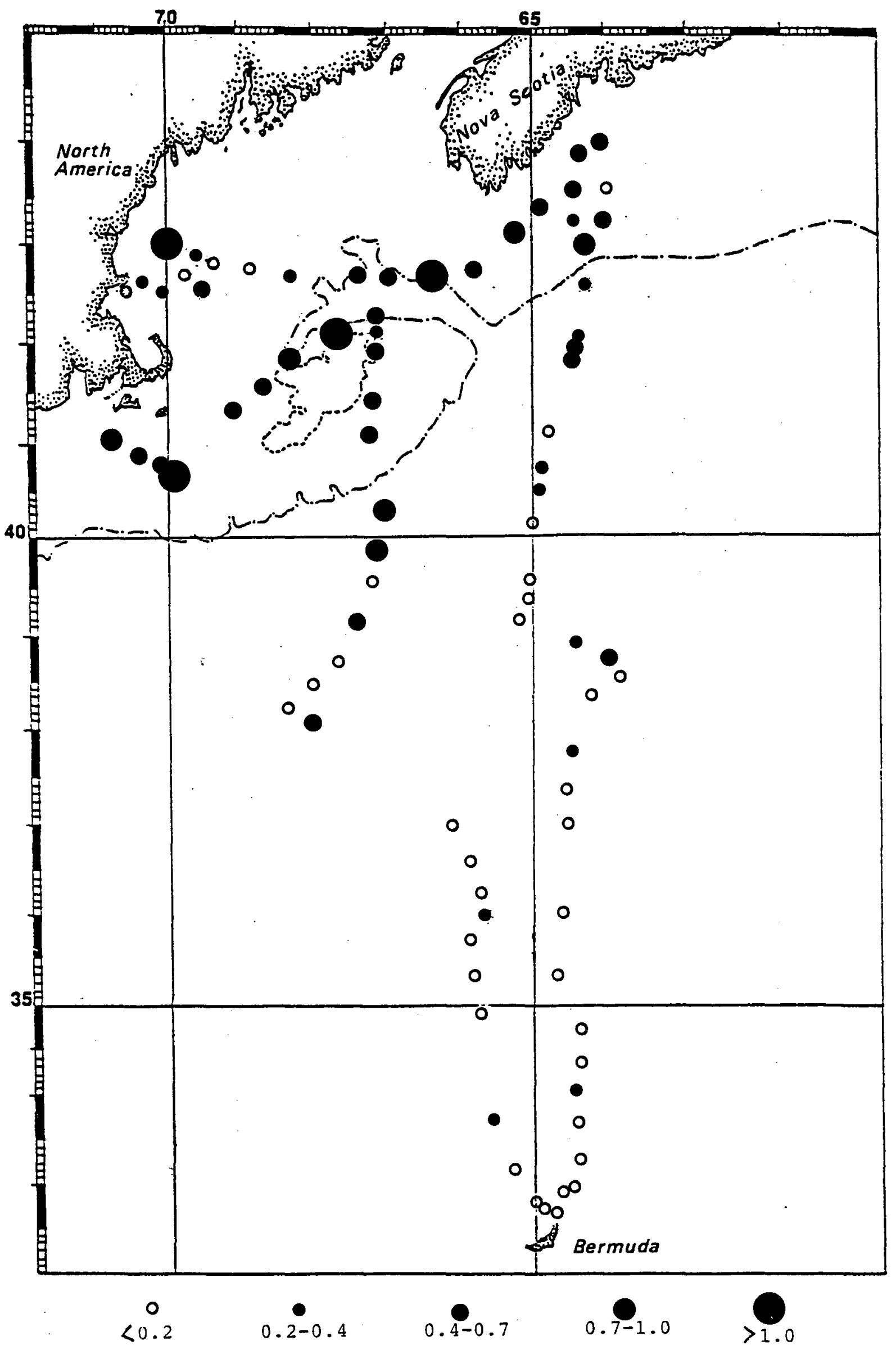

Figure 5 Horizontal distribution of dissolved inorganic phosphate for SSV Corwith Cramer Cruise C-112 $\left(\mathrm{mM} \mathrm{m}^{-3}\right)$. 


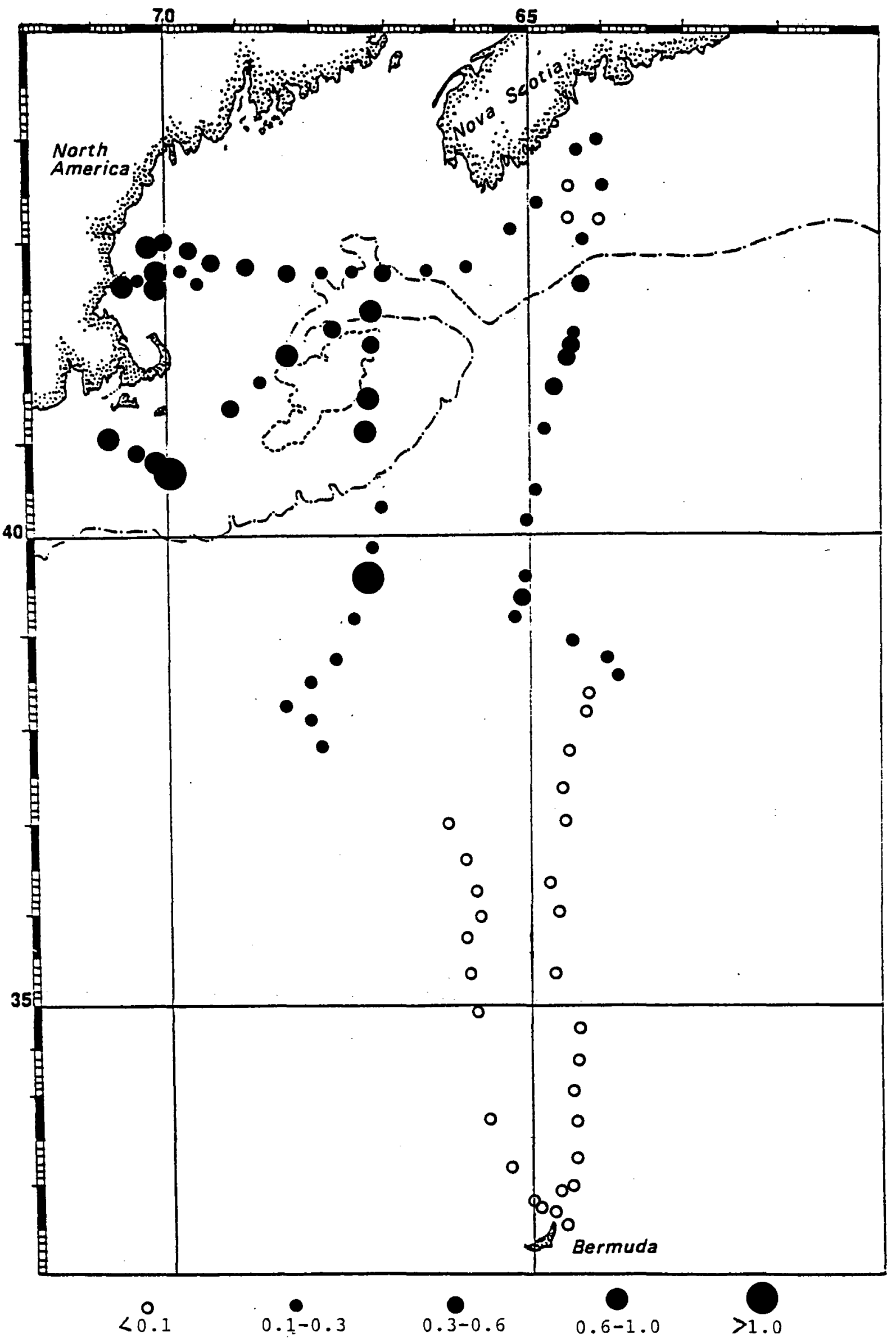

Figure 6 Horizontal distribution of chlorophyll a for SSV Corwith Cramer Cruise C-112 (mg m ${ }^{3}$ ). 
Table XI. Mean and Standard deviation for temperature, salinity, dissolved inorganic phosphate and chlorophyll in different oceanographic regions from surface samples taken during C-112

Region

Nantucket Shoals and Georges Bank SS1-10, HC-4, 9, 10

Warm Core Eddy SS11, 12

Slope Water

SS13-19, 46-53

HC-37, 40

Sargasso Sea

SS20-44

HC-32, 38, 39

Scotian Shelf

SS54-66

$\mathrm{HC}-48$

Gulf of Maine

SS67-73

HC-66

Western Gulf

of Maine

SS74-78, HC-62, 64
$\underline{T\left({ }^{\circ} \mathrm{C}\right) \quad \mathrm{Sal}(\%)}$

$8.2 \pm 0.7$

$\mathrm{n}=13$
$32.90 \pm 0.16$
$n=13$

$21.2 \pm 1.3$

$\mathrm{n}=2$

$\underset{n=2}{36.26 \pm 0.23}$
$0.88 \pm 0.13$
$\mathrm{n}=2$

$0.70 \pm 0.40$
$\mathrm{n}=13$

$0.68 \pm 0.30$

$\mathrm{n}=12$

$\mathrm{PO}_{1}\left(\mathrm{mM} \mathrm{m}^{-3}\right) \quad \mathrm{Chl}\left(\mathrm{mg} \mathrm{m}^{-3}\right)$

$$
\underset{n=2}{0.16 \pm 0.03}
$$

$$
\begin{array}{cccc}
18.6 \pm 1.9 & 35.21 \pm 0.51 & 0.22 \pm 0.19 & 0.23 \pm 0.13^{*} \\
\mathrm{n}=17 & \mathrm{n}=17 & \mathrm{n}=15 & \mathrm{n}=15
\end{array}
$$

$$
\begin{array}{cc}
22.8 \pm 1.1 & 36.58 \pm 0.18 \\
\mathrm{n}=29 & \mathrm{n}=29
\end{array}
$$
$0.11 \pm 0.12$
$\mathrm{n}=26$
$0.06 \pm 0.05$
$\mathrm{n}=28$
$0.54 \pm 0.27$
$\mathrm{n}=14$
$0.20 \pm 0.11$
$\mathrm{n}=14$

*SS13 excluded as an outlier.
$13.0 \pm 0.8$
$32.73 \pm 0.32$
$0.32 \pm 0.23$
$0.30 \pm 0.04$
$\mathrm{n}=8$
$\mathrm{n}=8$
$\mathrm{n}=7$
$14.2 \pm 1.2$
$\mathrm{n}=7$
$31.55 \pm 0.37$
$\mathrm{n}=6$

$.54 \pm 0.27$
$n=7$ 


\section{Comparative Plankton Dynamics of Georges Bank and the Scotian Shelf}

The difference in plankton dynamics between a vertically mixed continental shelf ecosystem and a stratified shelf system was investigated by comparing a station on Georges Bank (HC-11, 13) with one on the Scotian Shelf (La Have Bank) (HC-48, 49). A simple nutrient-phytoplankton-zooplankton food web was constructed by measuring the concentration of dissolved inorganic phosphate, chlorophyll, and zooplankton biomass using bottle casts and net tows and measuring photosynthesis, zooplankton grazing and zooplankton phosphate excretion using incubations and the phaeopigment gut content of the zooplankton. The complete methods will not be presented here. The photosynthesis was determined via in situ light and dark bottle incubations at selected light levels by measuring oxygen evolution. The zooplankton phosphate excretion measurements involved 8-10 hour incubations of zooplankton captured by nets in the morning and evening. Zooplankton grazing was determined by gut content analysis of animals taken from the same tows. The food web resulting from these measurements is presented in Figure 7 with all values integrated to the depth of the mixed layer which is to the bottom $(42 \mathrm{~m})$ on Georges Bank and at $41 \mathrm{~m}$ on La Have Bank (bottom depth $85 \mathrm{~m})$.

Phytoplankton abundance is much lower on the Scotian Shelf and no net photosynthesis was measured (it was very foggy) even though nutrient levels were the same (as mentioned in the previous section). Net photosynthesis on Georges Bank of $1332 \mathrm{mg} \mathrm{C} \mathrm{m}^{-2}$ day $^{-1}$ is similar to other reported values and results in a turnover rate of 1.1 for the algal biomass. On the other hand, zooplankton biomass and nutrient excretion are higher on the Scotian Shelf than on Georges Bank. This makes nutrient cycling more significant on the stratified Scotian Shelf than on the vertically well-mixed Georges Bank. Phosphate excretion by zooplankton supplies only $3 \%$ of the phosphate uptake corresponding to the net photosynthesis measured on Georges Bank. Grazing pressure is only $7.5 \%$ per day while phytoplankton are growing at more than $100 \%$ per day. These data support the view that Georges Bank must be receiving a continual input of exogenous nutrients (or else have very high benthic and microbial regeneration) and that, relatively speaking, is depauperate in macrozooplankton. 
Figure 7. Plankton dynamics of Georges Bank and the Scotian Shelf.

GEORGES BANK

NUTRIENTS

ZOO

\section{PHYTO}

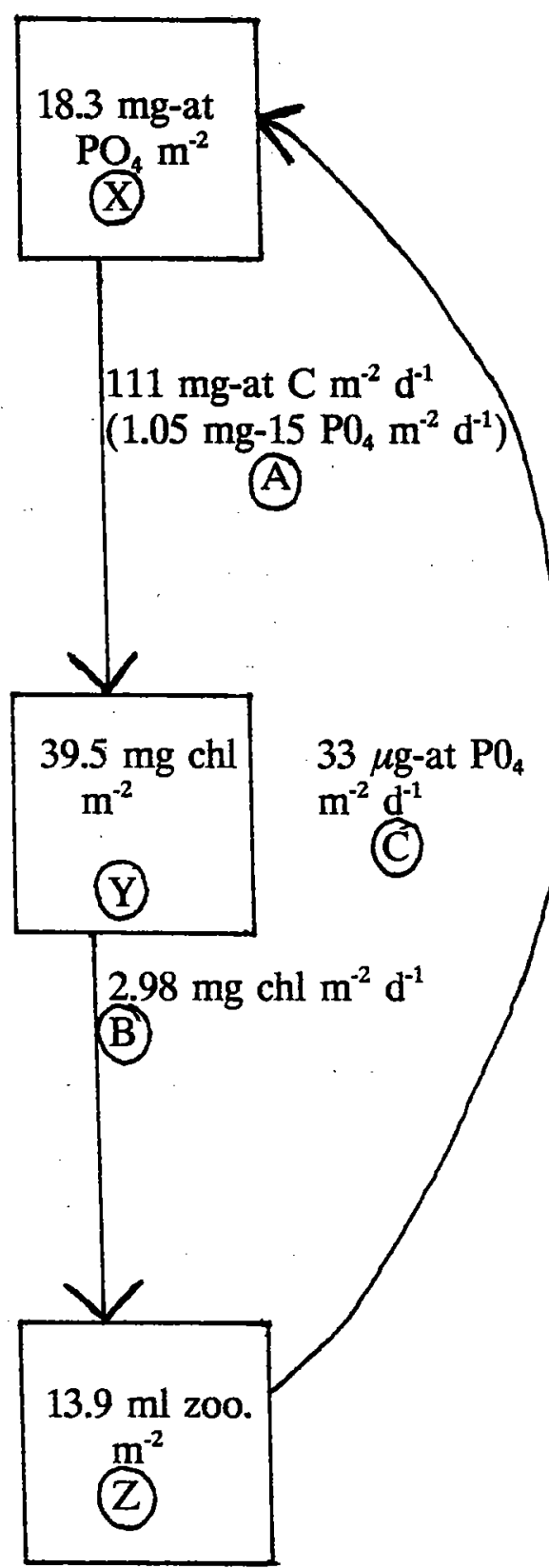

SCOTIAN SHELF

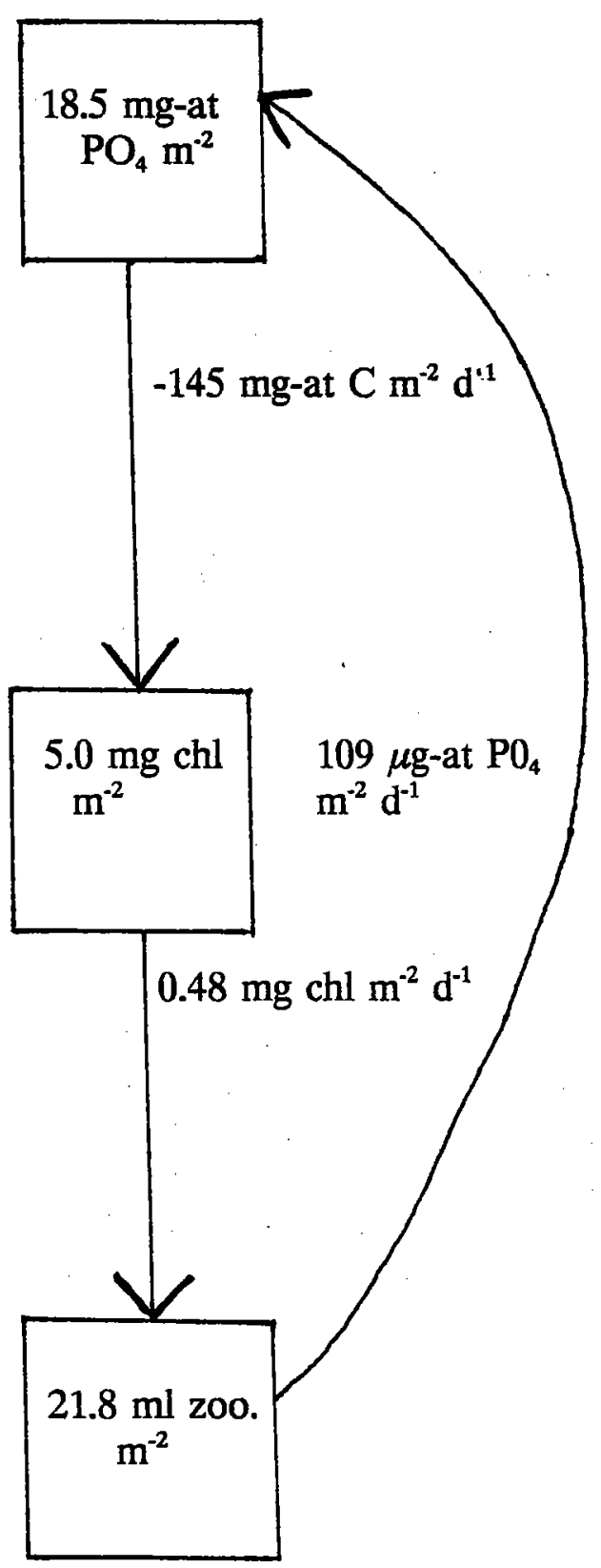

Conversion factors

$\mathrm{C}: \mathrm{P}=106: 1$ (atomic)

$\mathrm{C}: \mathrm{chl}=30: 1$ (weight)

ml zoopl.: $C$ (weight $)=29: 1$
Ratios:

Turnover of phyto $(\mathrm{A} / \mathrm{Y})$

Recycling by zoo (C/A)

Grazing pressure (B/Y

Ingestion per body wt. $(B / Z)$

Excretion per body wt. $(\mathrm{C} / \mathrm{Z})$
G.B. S.S.

1.100

$3 \%>100 \%$

$7.5 \% \quad 9.6 \%$

$18.7 \% \quad 1.9 \%$

$9 \% \quad 18 \%$ 


\section{Georges Bank Tidal Front}

Tidal fronts (also known as shallow sea fronts) represent the boundary between vertically mixed shallow areas and stratified (warmer water over colder water) deeper areas. The mixing is caused by tidal currents and the position of the front is determined by the strength of the currents and the depth of the water. These fronts have been found to be areas of enhanced biomass and biological activity in shallow seas. The process responsible for this increased activity include the diurnal and lunar changes in the position of the front and frontal upwelling.

The central area of Georges Bank is vertically mixed by the tides so that a tidal front exists surrounding it at about $60-80 \mathrm{~m}$ depth. The position of the front should be considered a broad band since the front moves approximately $10 \mathrm{~km}$ with the ebb and flood of the tide. On the northern flank of Georges Bank the front also corresponds with the edge of the bank and this produces some special interactions (specifically the generation of internal waves) that make this area particularly productive. The northern flank is known to have the highest abundances of seabirds and whales and is the only part of Georges Bank where diatoms (a group of algae associated with the spring bloom of phytoplankton and productive areas in general) remain in abundance throughout the summer. We investigated the tidal front in this area by measuring temperature, salinity, phosphate, and chlorophyll distributions at six stations across the front (Figure 8).

The tidal front was located between stations 4 and 5 off the edge of the bank in deep water (temperature, salinity and density distributions shown in Figures 9, 10 and 11). This probably corresponds to its maximum off bank location as the predicted low tide occurred at 1500 and stations 5, 6 and 7 were done at 1350, 1510 and 1620 respectively. The geostrophic current had maximum velocities at the front at depths from $25-40 \mathrm{~m}$ (Figure 12). The maximum of $15 \mathrm{~cm} \mathrm{sec}^{-1}$ is only about half the velocity measured by current meters in this region showing the importance of non-geostrophic factors. Some upwelling may be occurring at stations 5 and 6 . This is shown by the temperature and density distributions and the high phosphate concentration at about 30 $\mathrm{m}$ found at these stations (Figure 13). The chlorophyll distributions showed a subsurface maximum at the stratified off bank station and a vertically homogeneous distribution on the bank (Figure 14). At the front (station 5) the chlorophyll concentration was high and also extended quite deep in the water column (possibly as a result of vertical mixing). Another area of high chlorophyll was the bottom at the bank edge (station 7, Figure 14). A similar high chlorophyll region has been found in other studies and it may represent a convergence in the bottom currents. It coincided with an area of low phosphate concentration (Figure 13).

In summary, the location of the front was seaward of the edge of the bank corresponding to the tidal cycle at the time of sampling. Some evidence for upwelling and enhanced biological production was found in the high phosphate and chlorophyll levels at the front but an increased vertical mixing was also indicated. 


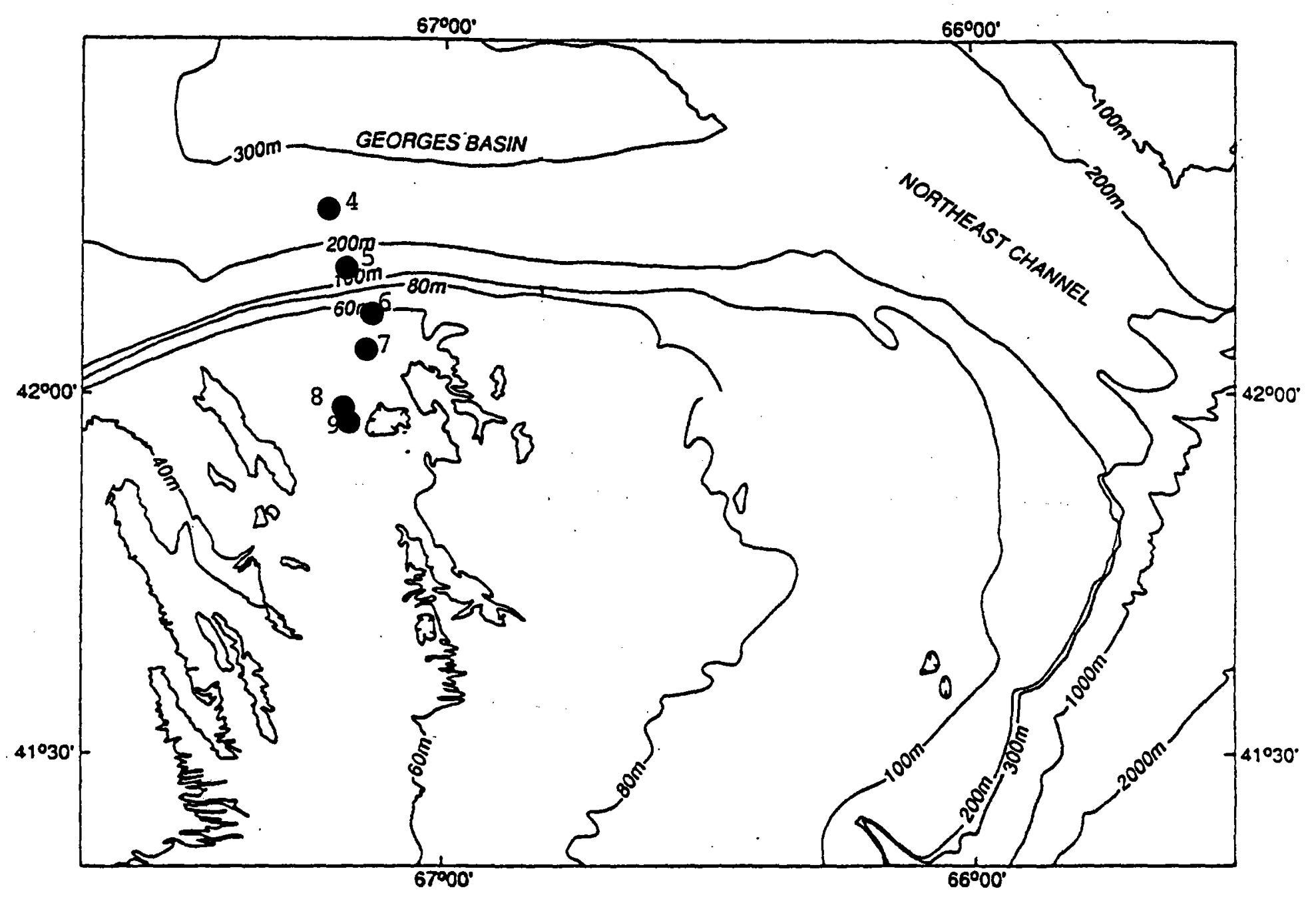

Figure 8 Sampling stations for Georges Bank tidal front transect. 
Georges Bank Tidal Front: Temperature

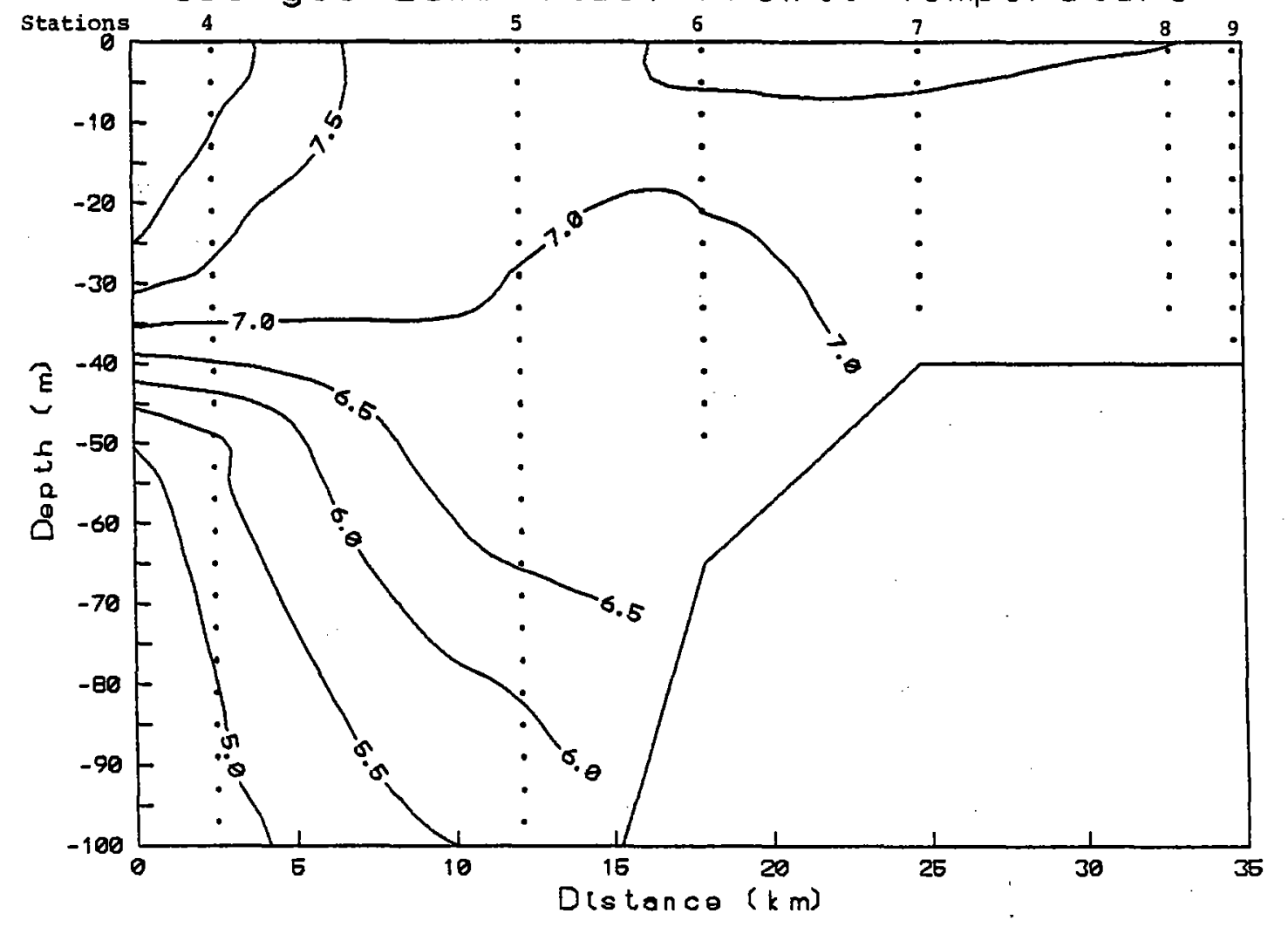

Figure 9

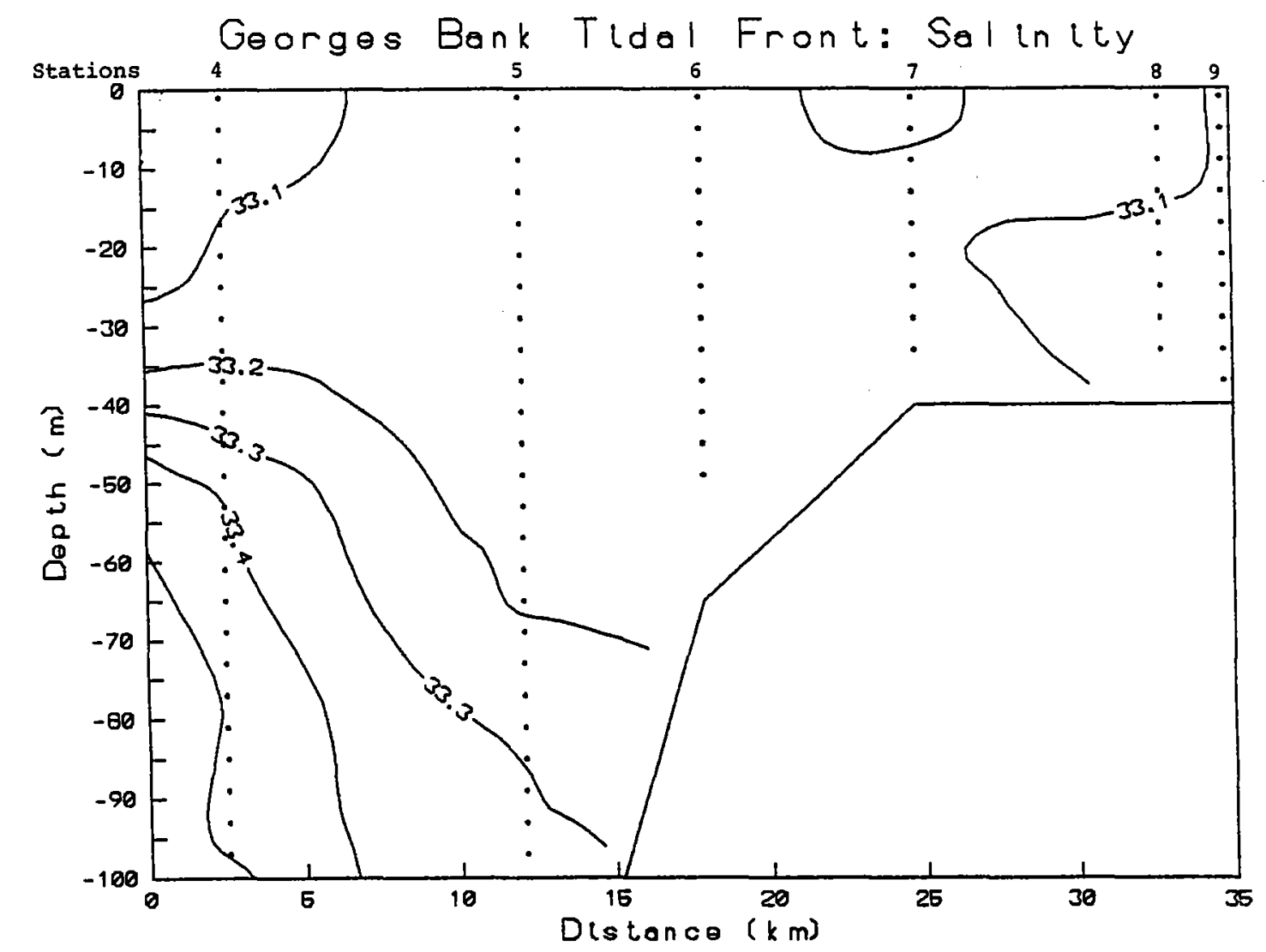

Figure 10 
Georges Bank Tidal Front: Denslty

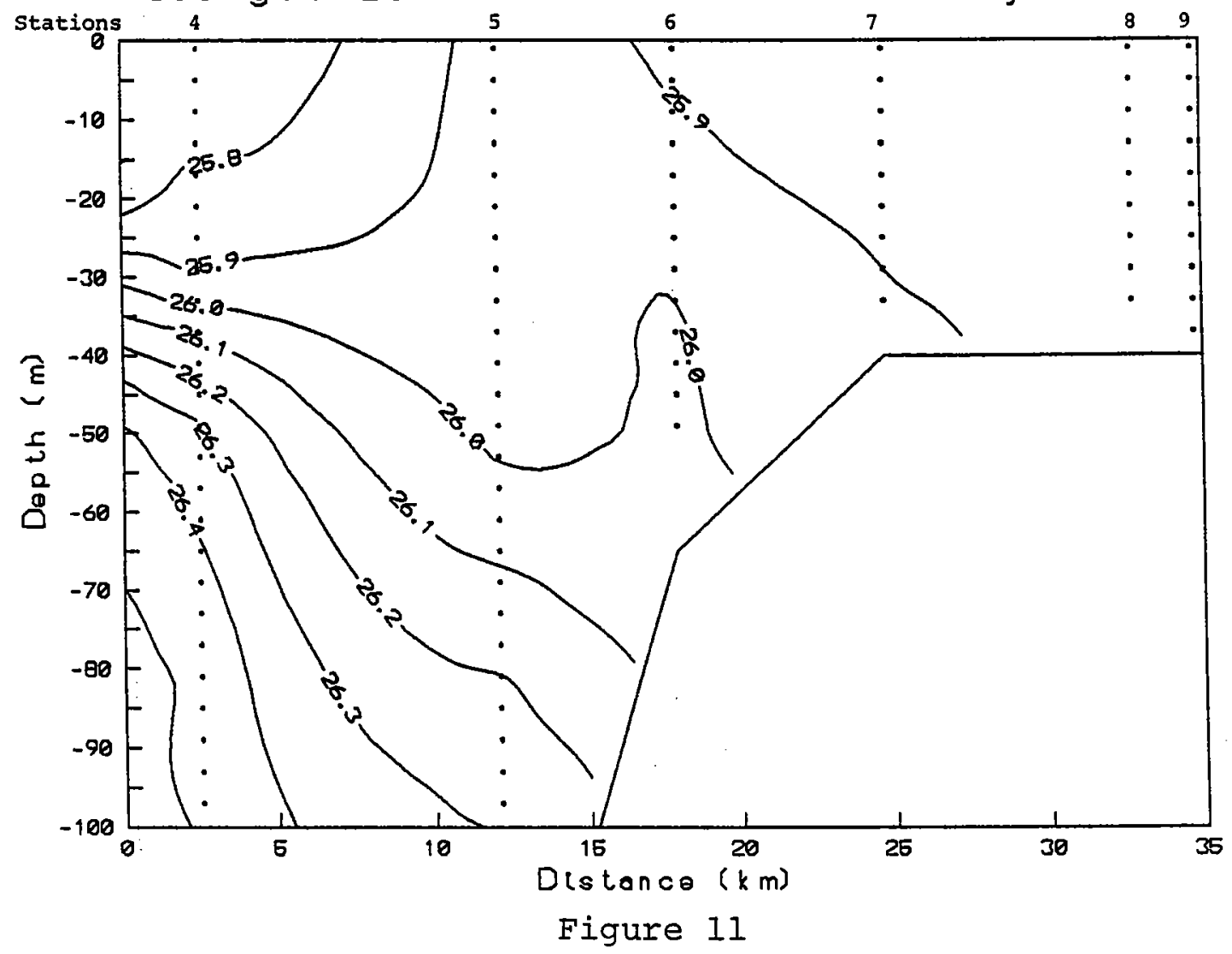

Georges Bank Tidal Front: Velocity $(\mathrm{m} / \mathrm{s})$

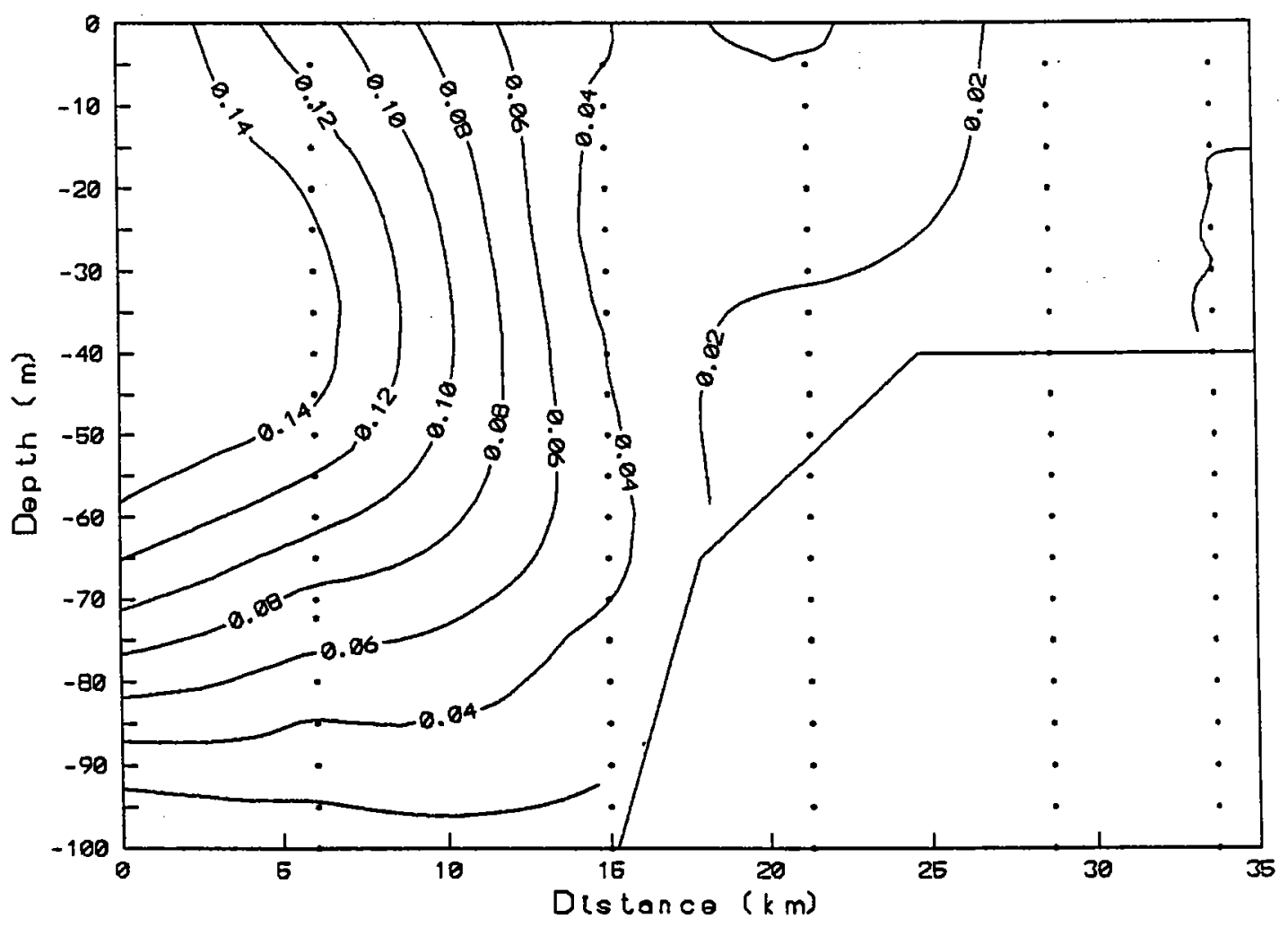

Figure 12 
Georges Bonk Tldal Front: Phosphote

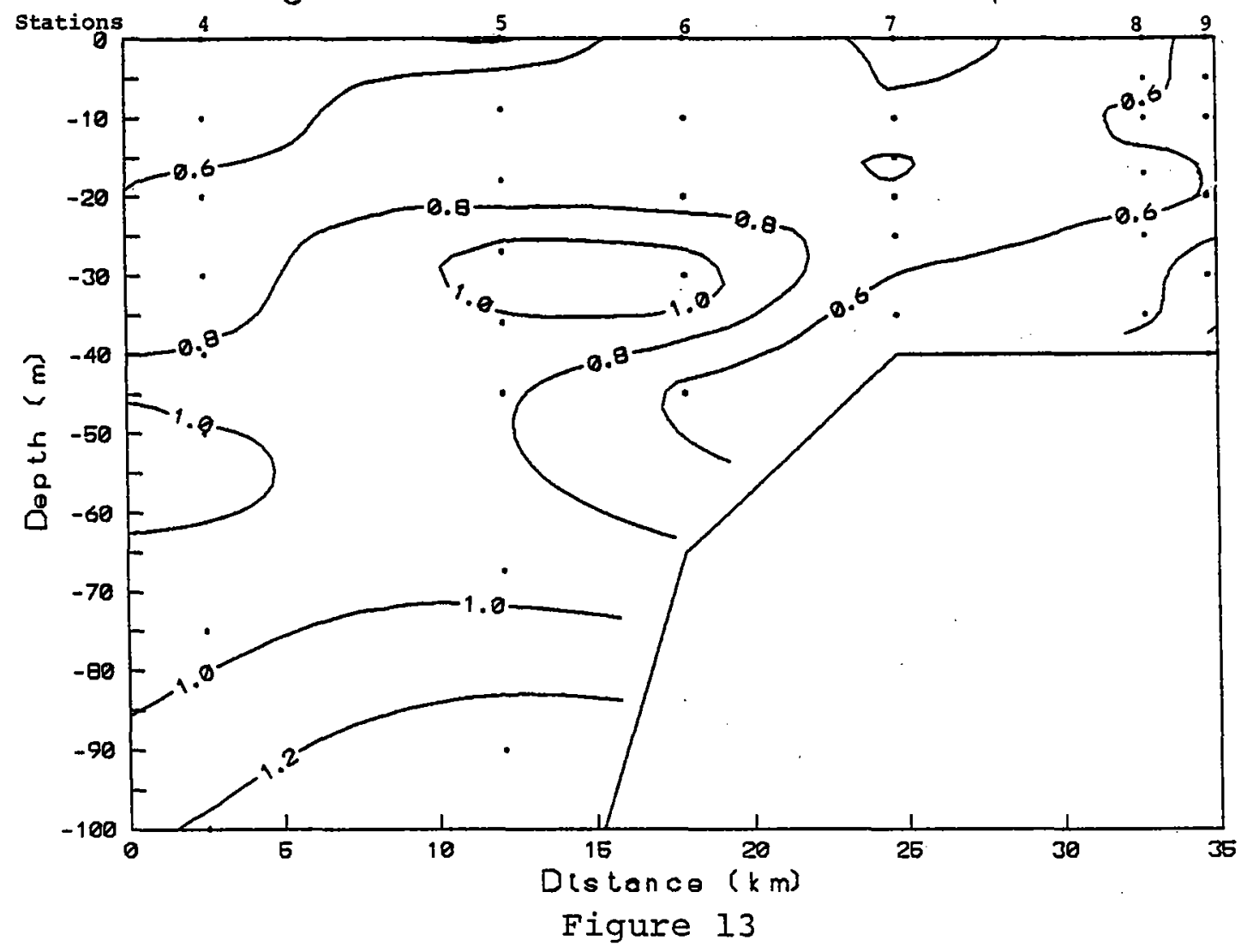

Georges Benk Tidal Front: Chlorophyl. I

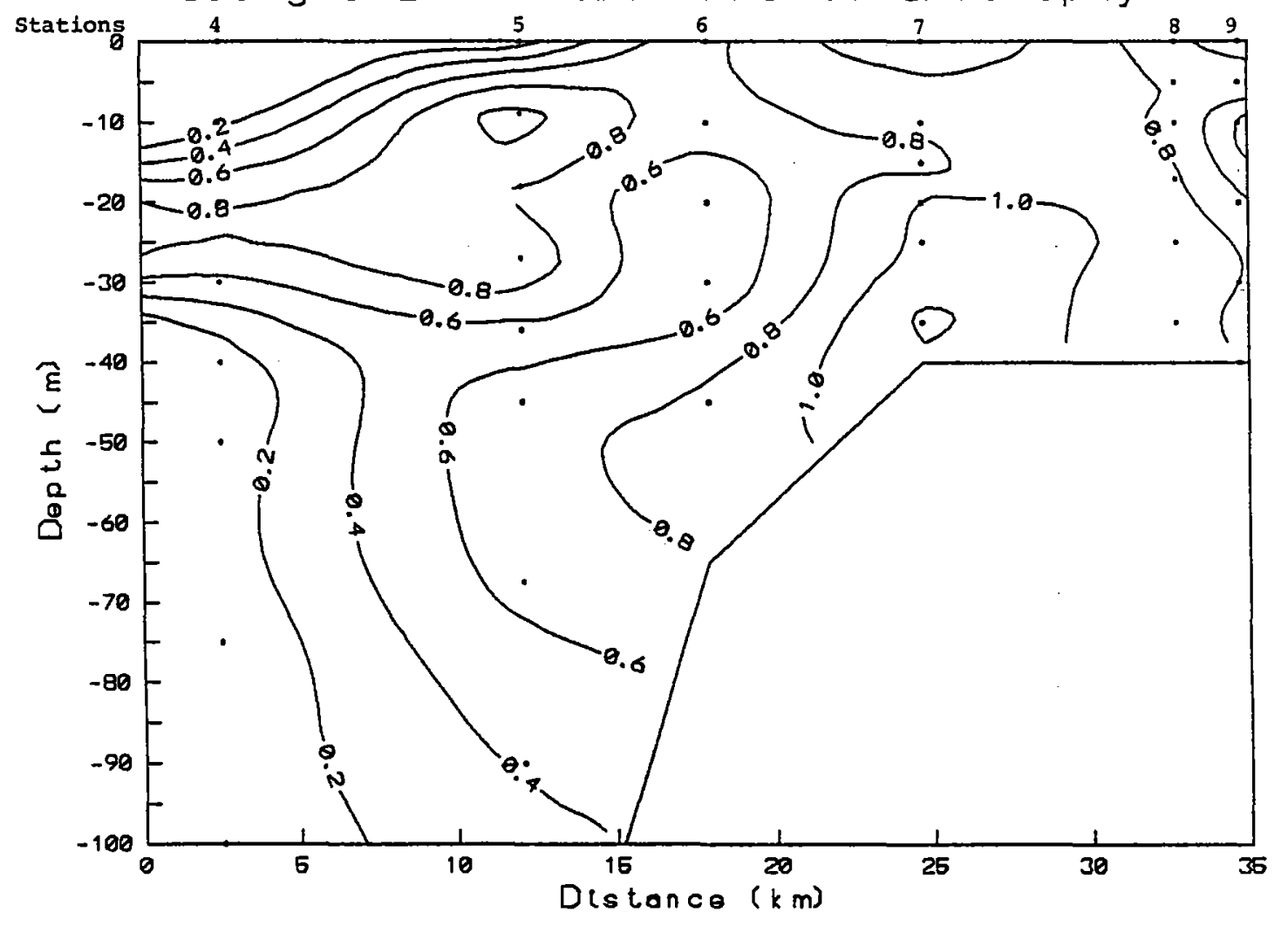

Figure 14 


\section{Gulf Stream Front}

The western and northern side of the Gulf Stream south of New England represents another kind of oceanic front known as a western boundary current front. In this area it separates Slope Water from the more saline Sargasso Sea Water. The Gulf Stream itself is part of the large-scale North Atlantic circulation. It is an extremely energetic system with a water transport of $80-100 \times 10^{6} \mathrm{~m}^{3} \mathrm{sec}^{-1}$ (enough to fill the Gulf of Maine in less than a day) and a huge heat transport (equivalent to the annual energy consumption of the United States). It has also been shown recently to be extremely important for nutrient transport.

We investigated the Gulf Stream Front by measuring temperature, salinity, phosphate and chlorophyll at seven stations across the front (Figure 15). Due to adverse weather conditions, the stations were not obtained in a line crossing the Stream. For the purpose of presentation, a straight line transect across the Stream was assembled by measuring the distance of the stations from the north wall of the Gulf Stream obtained by satellite. Presented in this way, the stations, although differing quite a bit in latitude, are clustered in the vicinity of the front. We were interested in comparing this largescale front to the Georges Bank tidal front and looking for evidence of upwelling and increased biomass in it.

The distribution of temperature and salinity showed the disappearance of the warm and salty Sargasso Sea Water across the front (Figures 16 and 17). The depth of the $10^{\circ} \mathrm{C}$ isotherm decreased from $900 \mathrm{~m}$ to $300 \mathrm{~m}$. Nutrient isolines also rose about 600 meters (Figure 18). The property distributions agree with the satellite map in indicating the front between stations 35 and 38. These two stations also showed the highest chlorophyll abundance (Figure 19). Chlorophyll was extremely low in the Sargasso Sea (station 34) with a chlorophyll maximum at $90+\mathrm{m}$ while in the Slope Water (station 40) it was higher and the maximum occurred at $25 \mathrm{~m}$. However, at the frontal stations the chlorophyll concentration remained high from $25 \mathrm{~m}$ to $100 \mathrm{~m}$. This is reminiscent of the high levels of chlorophyll found at depth in the vicinity of the tidal front and may indicate similar processes of vertical mixing. More specifically, it agrees with models predicting downwelling on the downstream side of a meander crest. Also of interest is the larger nutrient maximum beneath the frontal stations than beneath the Sargasso Sea indicating some concentration or accumulation mechanism.

The geostrophic currents in the Gulf Stream were calculated from the density distribution determined at stations $34,35,36,37,38$, and 40 . The proximity of the central stations and the uncertainty in their exact relative positions can create substantial local errors in the geostrophic velocities. To present a somewhat smoothed picture, station 39 was not used in the geostrophic calculations. The reference level used (assumed level of no motion) was $1400 \mathrm{~m}$.

The density section, velocity section, dynamic height, and vertical velocity profiles are shown in Figures 20, 21, 22 and 23. The maximum velocities were $2 \mathrm{~m} \mathrm{sec}^{-1}$ in a relatively shallow frontal jet just inside the cold wall (Figures 21 and 22) corresponding 







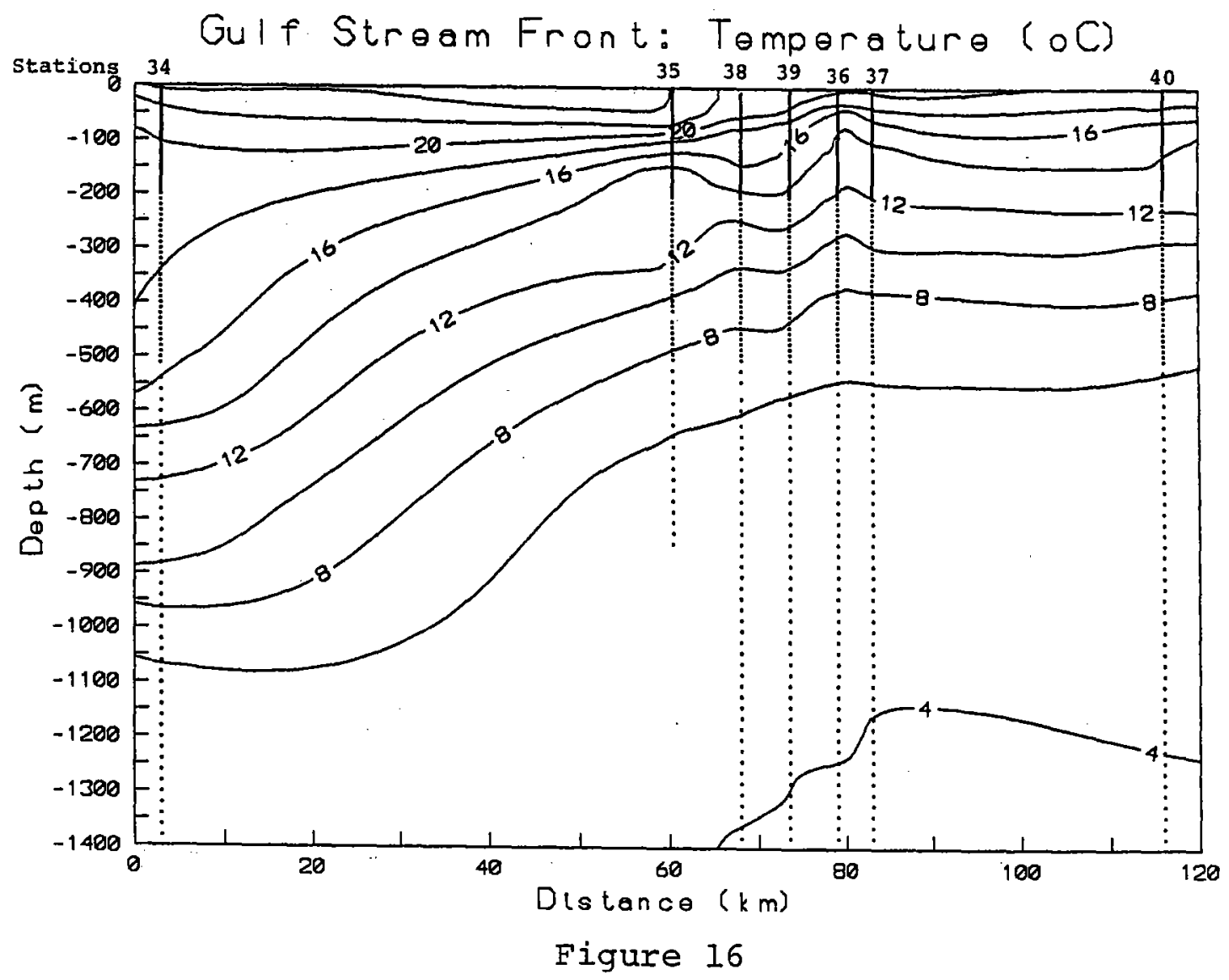

Gulf Stroam Front: $S a l$ (n (ty ( $p p t)$

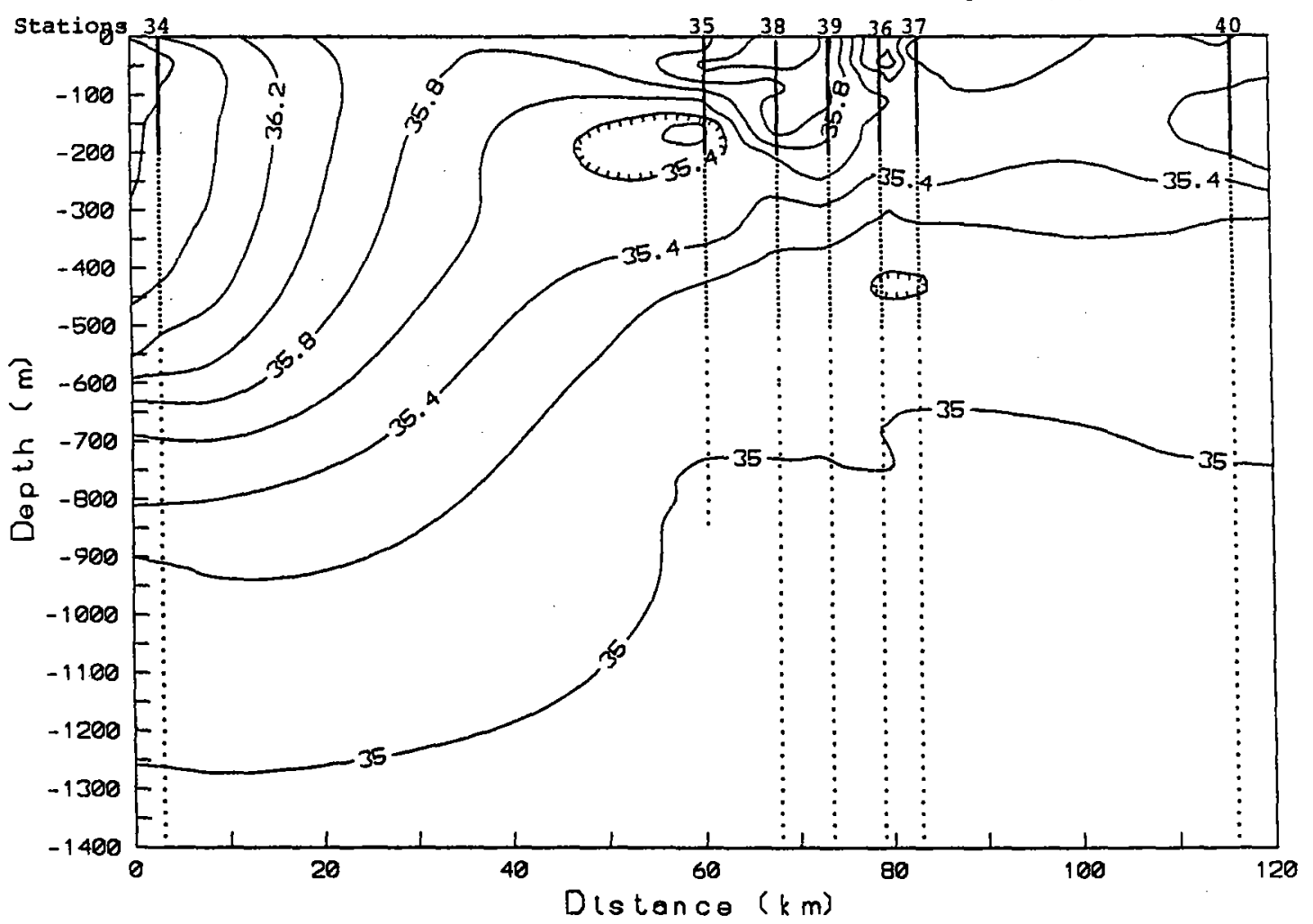

Figure 17 
Gulf Stroam Front: Phosphate (mM/m3)

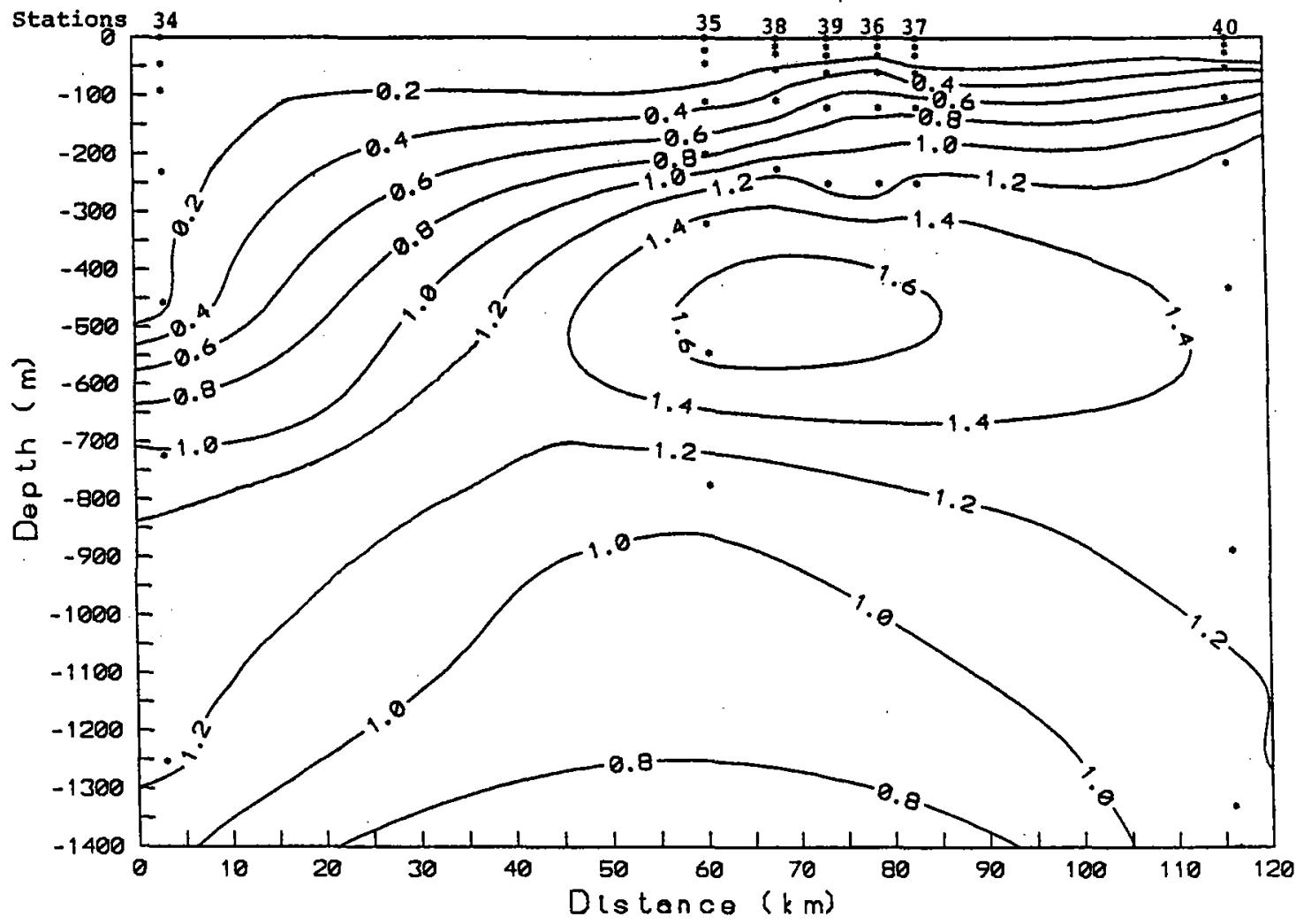

Figure 18

Gulf Stream Front: Chlorophyll ( $\mathrm{mg} / \mathrm{m} 3)$

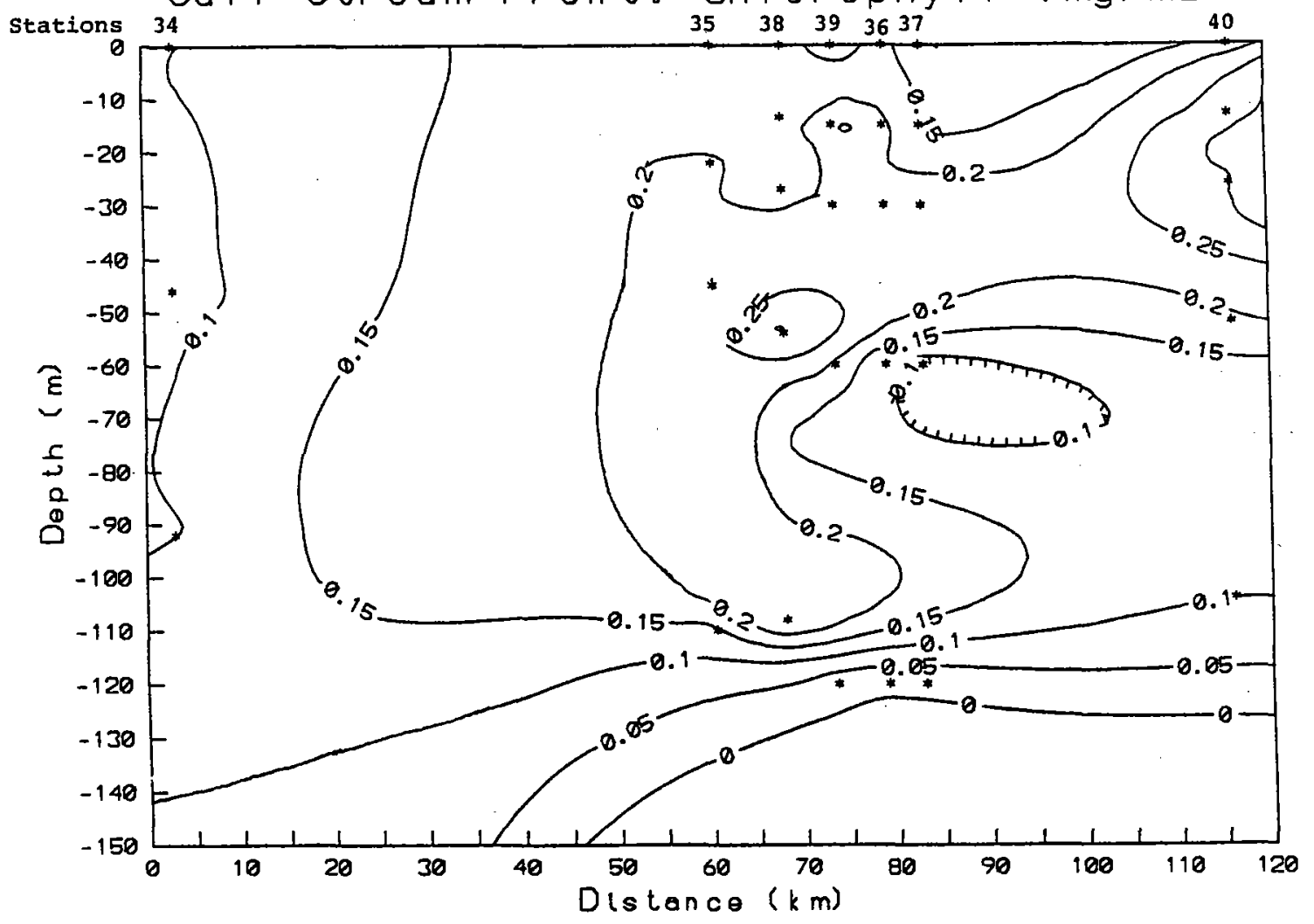

Figure 19 


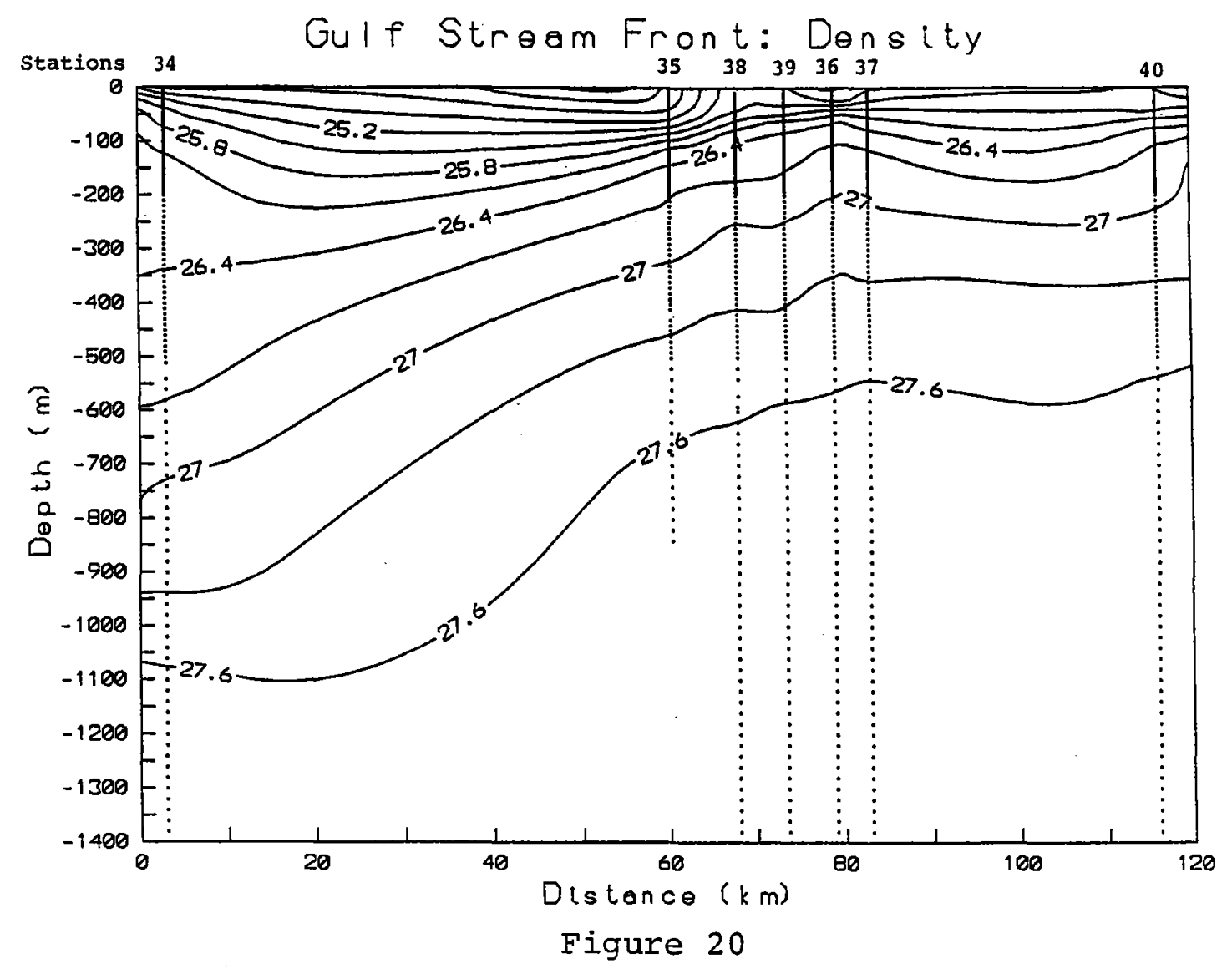

Gulf Stroam Front: $V_{\theta}$ loc (ty $(\mathrm{m} / \mathrm{s} \theta \mathrm{c})$

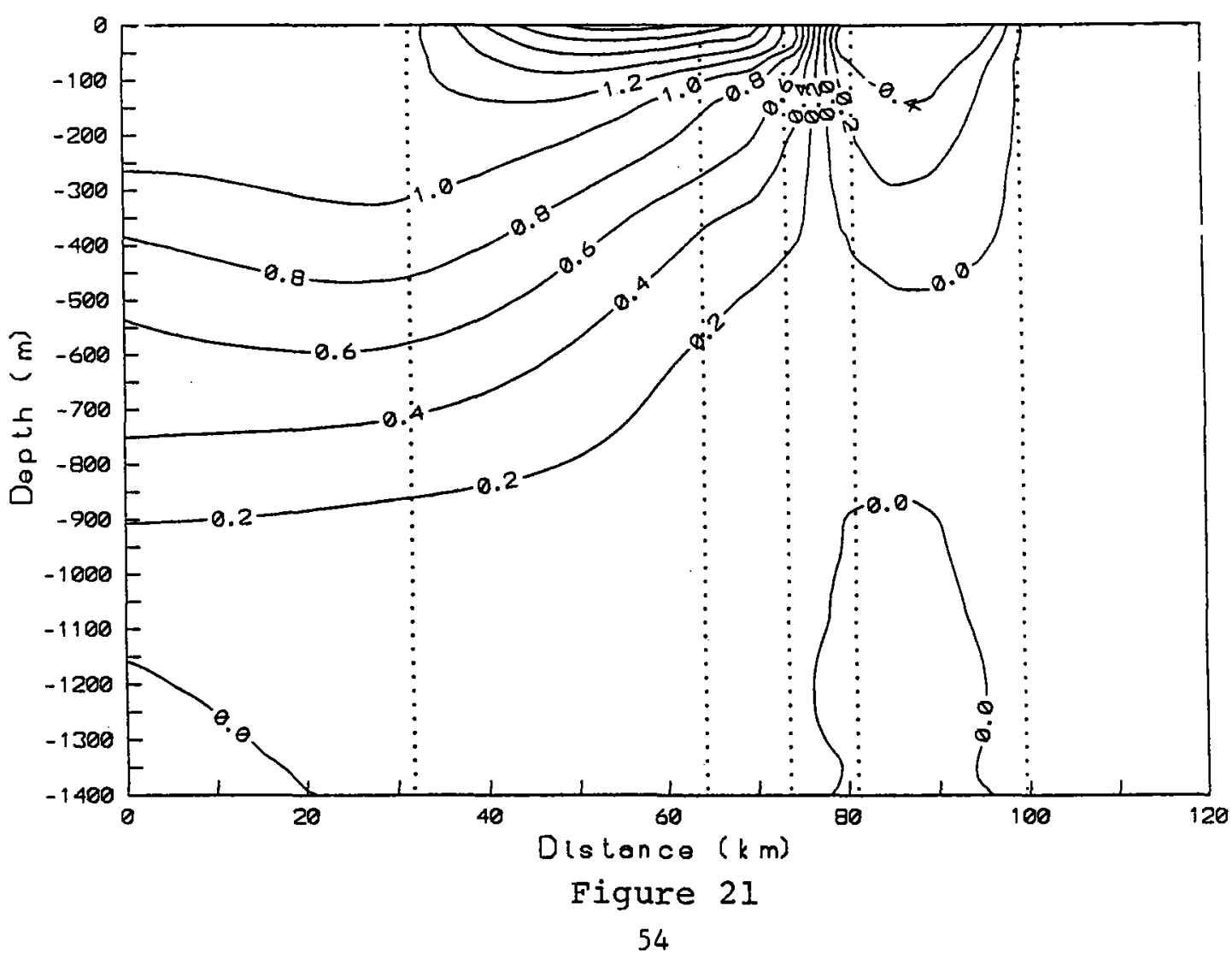




\section{Gulf Stream Velocity $(\mathrm{m} / \mathrm{sec})$}

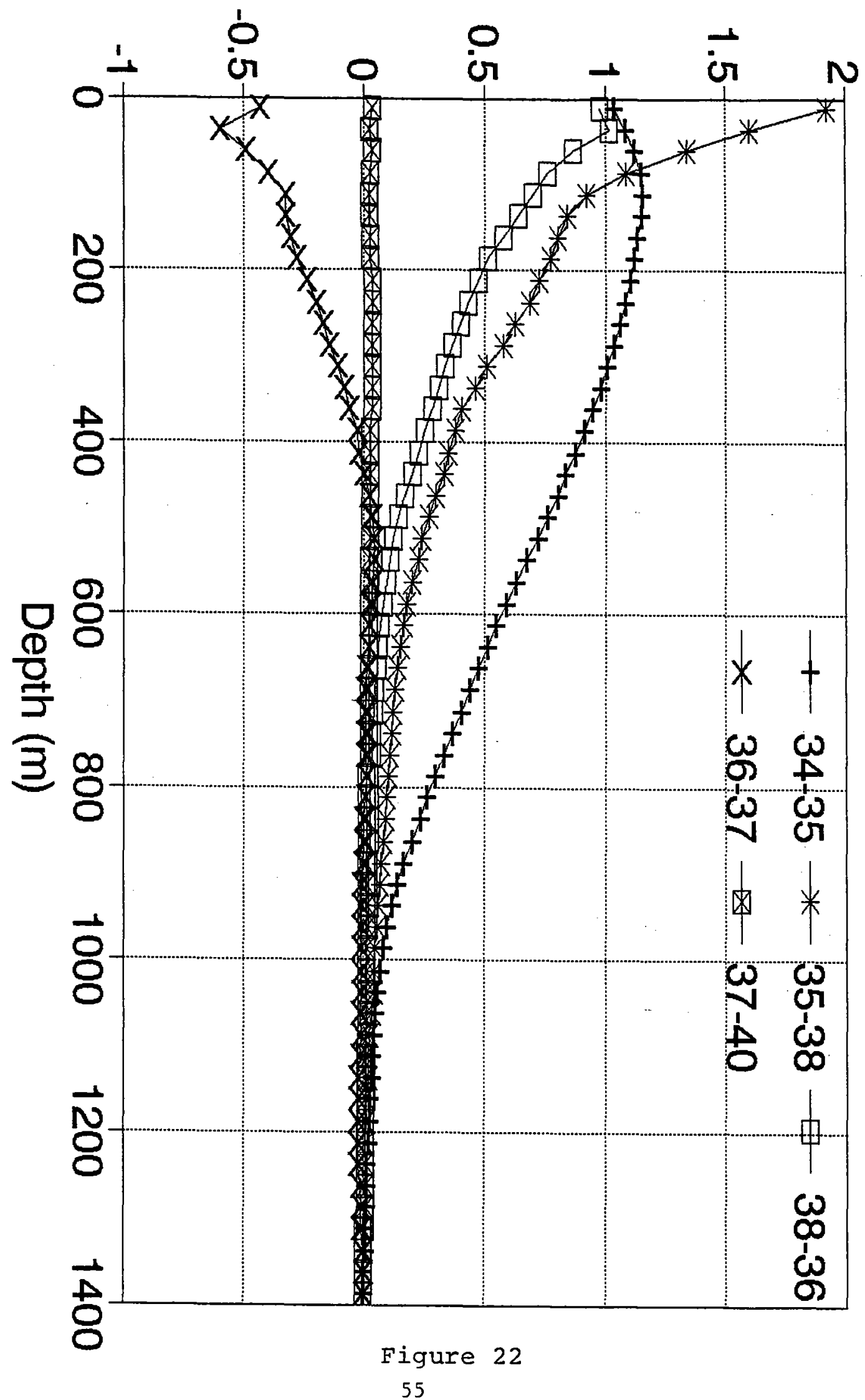




\section{Gulf Stream Front Dynamic Height}

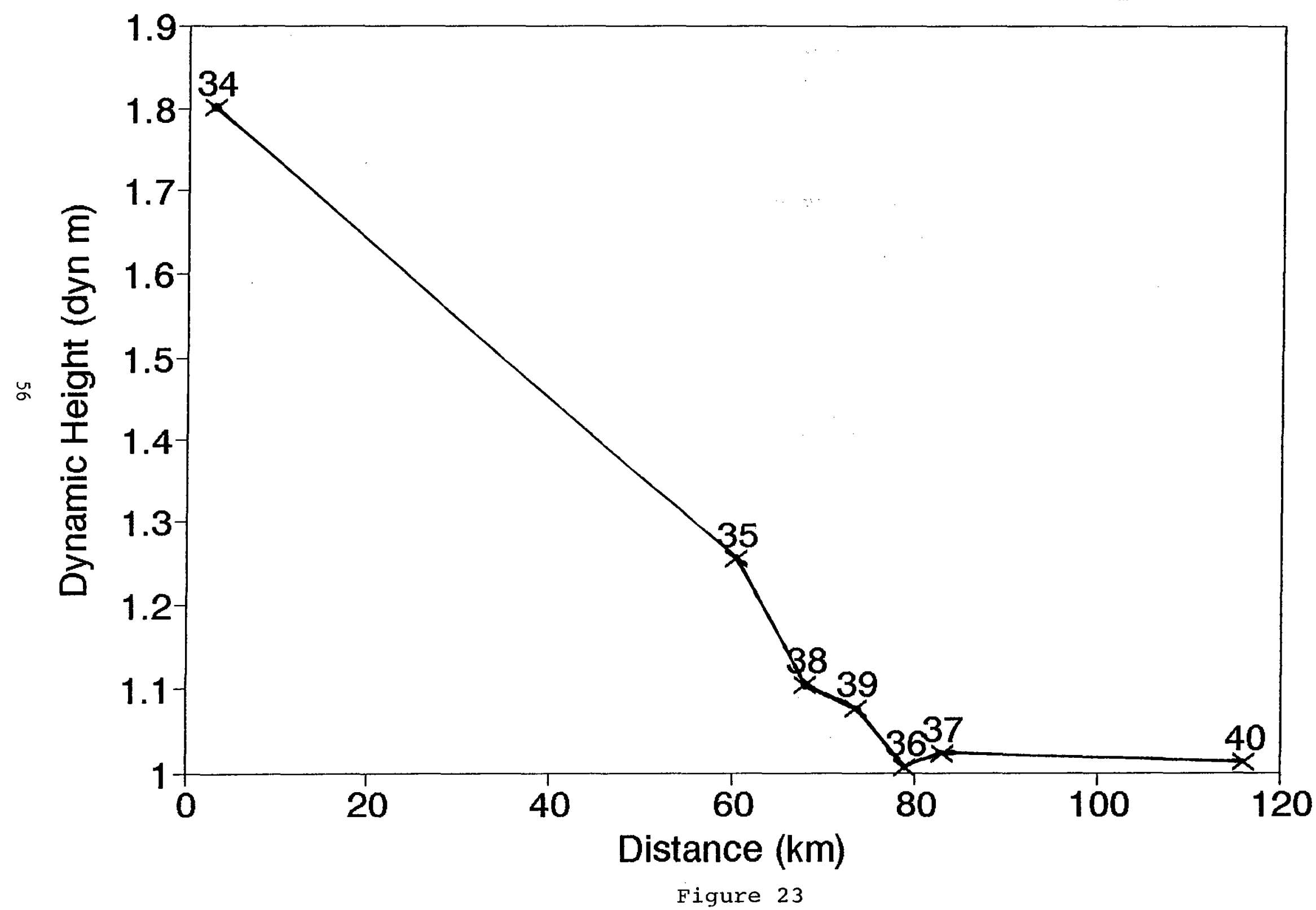


to the maximum spatial gradient in dynamic height (Figure 22). The maximum change in dynamic height was about $80 \mathrm{~cm}$. The Sargasso Sea boundary of the Gulf Stream was not determined. The length scale of the Gulf Stream, calculated by the equation:

$$
\lambda=9 / f\left(\Delta h_{m} / v_{m}\right) \text {, }
$$

where $g$ is the gravitational acceleration, $f$ is the Coriolis parameter, $h_{m}$ is the maximum height change across the anomaly and $v_{m}$ is the maximum along front geostrophic flow, was $42 \mathrm{~km}$. The dynamic heights of the larger area of our cruise track are shown in Figure 24 using $1000 \mathrm{~m}$ as the reference level. The decrease toward Bermuda is a feature of the recirculation in the Sargasso Sea.

\section{Plastic and Tar in the Northwest Atlantic Ocean}

The distribution and abundance of pelagic plastic and tar was determined by collections made along the cruise track with the neuston net. The plastic are counted as either pellets or pieces and the tar is weighed. Almost no tar or plastic was found outside the Sargasso Sea (stations HC-21-34) (Figures 25 and 26). This corresponds with the previous finding of SEA researchers that the presence of these pollutants was greatest in the large scale convergence system of the Sargasso Sea. 


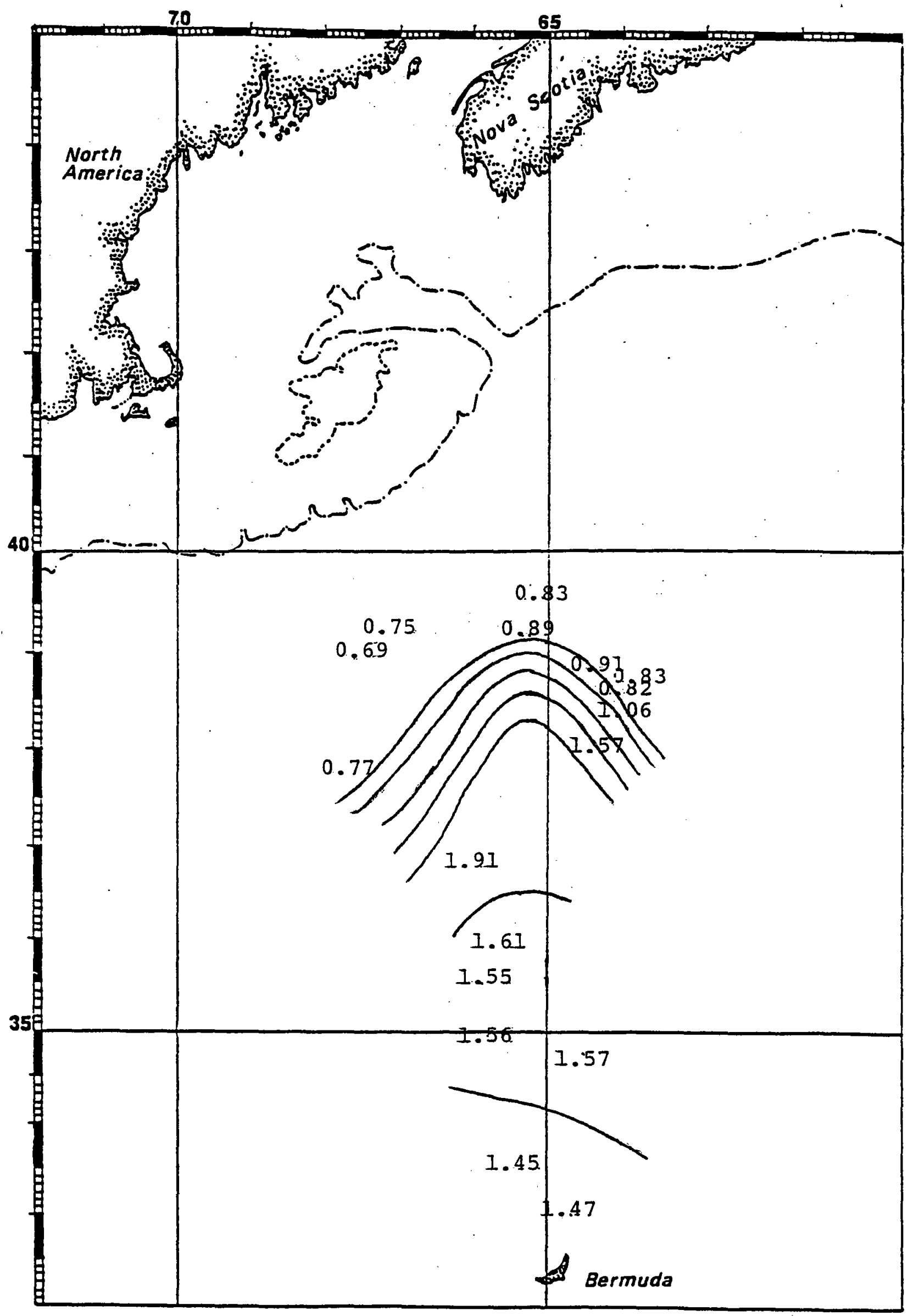

Figure 24 


\section{C112 Plastic Distribution}

(labelled by station)

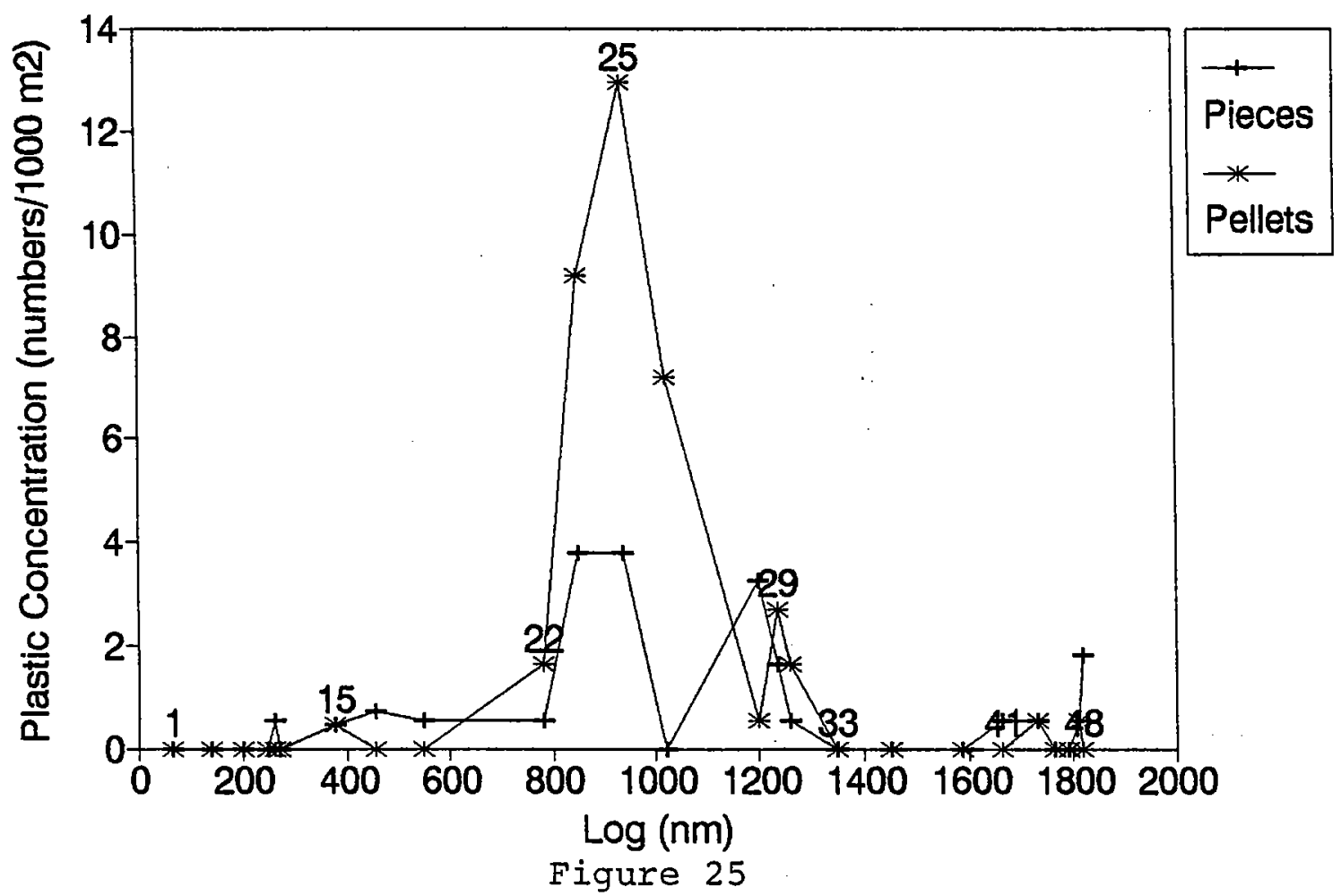

\section{C112 Tar Distribution (labelled by station)}

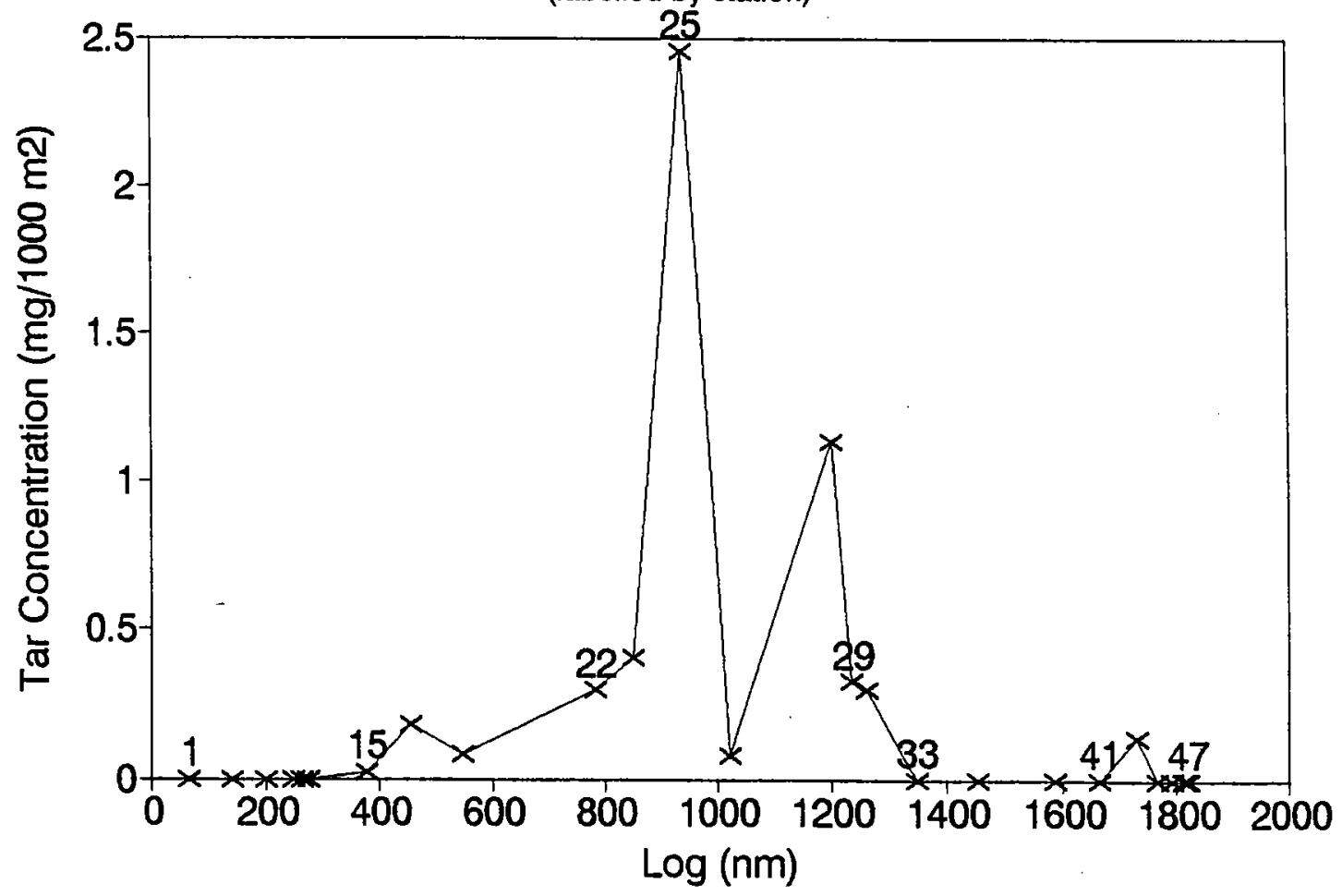

Figure 26 